University of Nebraska - Lincoln

DigitalCommons@University of Nebraska - Lincoln

2003

\title{
Multiphoton detachment of a negative ion by an elliptically polarized, monochromatic laser field
}

\author{
N. L. Manakov \\ Voronezh State University, Universitetskaya pl. 1, Voronezh, 394006 Russia, manakov@phys.vsu.ru
}

M. V. Frolov

Voronezh State University, Russia, frolov@phys.vsu.ru

Bogdan Borca

University of Nebraska-Lincoln

Anthony F. Starace

University of Nebraska-Lincoln, astarace1@unl.edu

Follow this and additional works at: https://digitalcommons.unl.edu/physicsstarace

Part of the Physics Commons

Manakov, N. L.; Frolov, M. V.; Borca, Bogdan; and Starace, Anthony F., "Multiphoton detachment of a negative ion by an elliptically polarized, monochromatic laser field" (2003). Anthony F. Starace Publications. 175.

https://digitalcommons.unl.edu/physicsstarace/175

This Article is brought to you for free and open access by the Research Papers in Physics and Astronomy at DigitalCommons@University of Nebraska - Lincoln. It has been accepted for inclusion in Anthony F. Starace Publications by an authorized administrator of DigitalCommons@University of Nebraska - Lincoln. 


\title{
Multiphoton detachment of a negative ion by an elliptically polarized, monochromatic laser field
}

\author{
N L Manakov ${ }^{1}$, M V Frolov $^{1,2}$, B Borca ${ }^{2,3,4}$ and Anthony F Starace ${ }^{2}$ \\ ${ }^{1}$ Department of Physics, Voronezh State University, Voronezh 394006, Russia \\ 2 Department of Physics and Astronomy, University of Nebraska Lincoln, \\ NE 68588-0111, USA \\ E-mail: manakov@phys.vsu.ru and astarace1@unl.edu
}

Received 3 July 2002, in final form 20 February 2003

Published 22 April 2003

Online at stacks.iop.org/JPhysB/36/R49

\begin{abstract}
The quasistationary quasienergy state approach (QQES) is applied to the analysis of partial ( $n$-photon) decay rates and angular distributions (ADs) of photoelectrons produced by an elliptically polarized laser field. The problem is formulated for a weakly bound electron with an energy $E_{0}$ in the threedimensional $\delta$-model potential (which approximates the short-range potential of a negative ion) interacting with a strong monochromatic laser field having an electric vector $\boldsymbol{F}(\omega t)$. The results presented cover weak (perturbative), strong (nonperturbative), and superstrong field regimes as well as a wide interval of frequencies $\omega$ extending from the tunnelling ( $\hbar \omega \ll\left|E_{0}\right|$ ) and multiphoton $\left(\hbar \omega<\left|E_{0}\right|\right)$ cases up to the high frequency domain $\left(\hbar \omega>\left|E_{0}\right|\right)$.

For a weak laser field, exact equations for the normalization factor and for the Fourier coefficients of the QQES wavefunction at the origin $(|r| \rightarrow 0)$ (that are key elements of the QQES approach for a $\delta$-model potential) as well as for the detachment amplitudes are analysed analytically using both standard RayleighSchrödinger perturbation theory (PT) in the intensity, $I$, of the laser field and Brillouin-Wigner PT expansions involving the exact (complex) quasienergy $\epsilon$. The lowest-order perturbative results for the $n$-photon ADs are presented in analytic form, and the parametrization of ADs in terms of polarization- and angular-independent atomic parameters is discussed for the general case of elliptical polarization. The major emphasis is on the analysis of an ellipticity induced distortion of three-dimensional ADs and, especially, on the elliptic dichroism (ED) effect, i.e. the dependence of the photoelectron yield in a fixed direction $\boldsymbol{n}$ on the sign of the ellipticity (or on the helicity) of a laser field. The dominant role of binding potential effects for a correct description of ED and threshold effects is demonstrated, and the intimate relationship between atomic ED factors and scattering phases of the detached electron is established for multiphoton detachment, including the above-threshold case.
\end{abstract}

\footnotetext{
3 Present address: JILA, University of Colorado and National Institute of Standards and Technology, Boulder, CO 80309-0440, USA.

4 On leave from: Institute for Space Sciences, Bucharest-Magurele 76900, Romania.
} 
For a strong laser field, we present an accurate derivation for the QQES wavefunction and decay rates in the Keldysh approximation (KA) from exact QQES equations, including analytical, first-order ('rescattering') corrections to the KA results. The symmetries of ADs and the existence of $\mathrm{ED}$ are established using the exact analytical result for the $n$-photon detachment amplitude. Accurate numerical results are presented for the variation of the structure of the ADs as well as of the ED effect with increasing laser intensity.

For the high frequency case, $\hbar \omega>\left|E_{0}\right|$, a rigorous analytical treatment of higher-order PT effects is presented for one-photon detachment, taking into account corrections of higher orders in $I$ to the well-known photodetachment cross section for a short-range potential. Together with the exact numerical analysis of the total and partial decay rates for $\hbar \omega>\left|E_{0}\right|$, these results demonstrate the existence of a quasistationary stabilization regime in the decay of a weakly bound electron for any polarization of the laser field. Moreover, this stabilization occurs only over a limited interval of intensity, up to the closure of the direct photodetachment channel.

In the superstrong field regime, the total decay rate of a weakly bound electron may be described by cycle-averaging the results for an instantaneous static electric field of strength $|\boldsymbol{F}(\omega t)|$ (for any laser frequency and polarization). All results in this paper are presented in scaled units and are illustrated numerically for the case of the $\mathrm{H}^{-}$negative ion.

\section{Contents}

1. Introduction $\quad 51$

1.1. Brief survey of dichroic effects in unpolarized atom photoprocesses 51

1.2. Status of multiphoton detachment of negative ions 55

1.3. Outline of this review 58

2. Basic results of the QQES approach for the $\delta$-model potential and general equations for multiphoton detachment rates

2.1. Definitions and scaled units

2.3. Basic equations for the QQES solution for a $\delta$-model potential 63

2.4. Exact result for multiphoton detachment rates 64

2.5. The QQES wavefunction and detachment rates in the Keldysh approximation 66

2.6. Analytical PT results for angular distributions and $n$-photon rates 67

3. Angular distributions and elliptic dichroism in the weak field limit 73

3.1. Perturbative analysis of angular distributions for elliptically polarized light $\quad 73$

3.2. Discussion of dichroic terms in ADs 78

3.3. Comparisons with recent experiments and multielectron calculations $\quad 81$

4. Strong field analysis of angular distributions and ED effects $\quad 84$

4.1. Symmetry properties of angular distributions and elliptic dichroism $\quad 84$

4.2. Total and partial rates of $n$-photon detachment $\quad 85$

4.3. Three-dimensional angular distributions $\quad 88$

4.4. Elliptic dichroism in a strong field 91

5. Photodetachment in a strong, high frequency field $(\omega>1) \quad 95$

5.1. Higher-order corrections to the one-photon detachment cross section 96

5.2. The quasistationary stabilization regime 99

5.3. Angular distributions and ED for $\omega>1 \quad 101$ 
6. Static-electric-field behaviour of decay rates in superstrong fields 102

6.1. General remarks 102

6.2. $\omega^{2}$ expansion for the complex quasienergy 103

6.3. Comparisons with numerical results 105

7. Summary and conclusions 107

$\begin{array}{ll}\text { Acknowledgments } & 108\end{array}$

Appendix A. Normalization of the QQES wavefunction for a $\delta$-model potential 109

Appendix B. Perturbative and strong field results for the coefficients $\phi_{n}$ and $f_{n} \quad 109$

$\begin{array}{ll}\text { B.1. Brillouin-Wigner expansions } & 110\end{array}$

B.2. Rayleigh-Schrödinger results 111

$\begin{array}{ll}\text { B.3. Exact strong field results } & 114\end{array}$

B.4. 'Rescattering approximations' for the coefficients $f_{n} \quad 117$

Appendix C. Details of derivations for the $n$-photon detachment amplitude 118

$\begin{array}{ll}\text { References } & 120\end{array}$

\section{Introduction}

Recent studies of laser-atom interactions have indicated a growing interest in the dependence of multiphoton processes on the polarization state of a laser field. At present, a considerable ellipticity dependence is well documented for a number of strong field processes (e.g. high harmonic generation (HHG), laser-assisted collisions and ionization, including abovethreshold ionization) both for single- and multicolour laser fields. Significant differences in the magnitude of laser-atom interactions for linearly and circularly polarized laser fields were predicted first in 1966 for the decay of a weakly bound atomic system in the tunnelling regime [1] (see also [2] for an extension of these results to the case of elliptical polarization). In the multiphoton regime, the differences between multiphoton ionization cross sections were discussed long ago on the basis of perturbative calculations (see, e.g., [3]). Also, perturbative results in this regime for an arbitrary laser ellipticity have been discussed in [4] (for ionization) and in [5] (for harmonic generation). In the past decade a new manifestation of polarization effects in laser-atom processes has been discussed: dichroic effects caused by the dependence of physical observables on the helicity of a laser field, i.e. on the sign of the degree of circular polarization $^{5}, \xi$. Moreover, in the multiphoton regime the manifestation of these effects is possible in two different ways, either as circular dichroism (CD) (which is most pronounced for $\xi= \pm 1$ ) or as elliptic dichroism (ED) (which occurs only for the case of an elliptical polarization, with $0<|\xi|<1$, and which is zero for $|\xi|=0,1)$. In this review we present a detailed analysis of laser detachment of a weakly bound atomic system (a negative ion) in both perturbative and nonperturbative regimes (in the laser intensity), including a general analysis of ED effects on the angular distribution of detached electrons and their dependence on the laser frequency and intensity. Since studies of dichroic effects in photoprocesses involving freely oriented (i.e. not oriented or aligned) atoms are relatively new and not commonly known, we give below a brief survey of existing results for these effects.

\subsection{Brief survey of dichroic effects in unpolarized atom photoprocesses}

The difference between cross sections of a photoprocess for right $(\xi=+1)$ and left $(\xi=-1)$ circular polarization of an incident photon beam, the $\mathrm{CD}$ effect, is a widely used tool

$5 \xi$ is defined in (5) below. It takes values in the range $-1 \leqslant \xi \leqslant 1$, where $\xi=-1,0,+1$ correspond to left circularly, linearly and right circularly polarized light, respectively. 
to investigate the linear response of magnetic solids and chiral molecular systems to an electromagnetic field [6]. Asymmetries in the interaction of polarized light with chiral molecular samples have been known since Pasteur's experiments on optical activity [7], and the theory of the effect has been reviewed [8]. Recently, direct measurements have been performed of $\mathrm{CD}$ in the photoelectron angular distributions (ADs) resulting from photoionization of free, randomly oriented chiral molecules [9].

The CD effect is also well known in VUV and soft-x-ray photoprocesses (e.g. photoionization) from polarized atomic targets and in photoionization experiments in which the spin of the photoelectron is measured [8, 10]. As follows from simple symmetry arguments, the CD for these cases (as well as for magnetic samples) is completely caused by the existence of a time-odd pseudovector inherent to the problem being analysed, say $\boldsymbol{A}$ (which might represent, e.g., an angular momentum for the case of a polarized atom, a photoelectron spin for the case of atomic photoionization or a magnetization vector for the case of a magnetic solid). In general, the existence of a CD effect means that the cross section of a particular process involves one or more terms proportional to $\xi$, the circular polarization degree. However, as may be seen from equation (5) below, $\xi$ is proportional to $\left(\hat{k} \cdot\left[e \times e^{*}\right]\right)$, where $\hat{k}$ is the unit vector in the direction of the (time-odd) photon wavevector $\boldsymbol{k}$ and $\boldsymbol{e}$ is the (in general complex) photon polarization vector. One thus sees that $\xi$ is a pseudoscalar (due to its change of sign upon coordinate inversion). It is also time-even, since $\hat{k}=\boldsymbol{k} / \boldsymbol{k}$ is time-odd and also [ $e \times e^{*}$ ] is time-odd (since $e$ and $e^{*}$ get interchanged under time inversion, i.e. $e \rightarrow e^{*}$ ). Moreover, the parameter $\xi$ can initially enter a cross section only through a combination of vectors $e$ and $e^{*}$ (that enter the problem through the operator for the photon-atom interaction). This combination is unique and has the form $\mathrm{i}\left[e \times e^{*}\right]=\xi \hat{k}($ see $(5))$, where $\xi \hat{k}$ is a time-odd pseudovector. Thus the fundamental object of a light beam responsible for dichroic effects is the 'CD vector', $\xi \hat{k}$, which is generic to any elliptically polarized photon (i.e. having $\xi \neq 0$ ). For atomic targets that are polarized (as well as for magnetic solids), a time-odd pseudovector of the problem, $\boldsymbol{A}$, arises naturally. It may represent, e.g., either a spin or an angular momentum. Since all terms contributing to any cross section must be true scalars, one concludes that, for polarized targets, the $\mathrm{CD}$ term arises simply as the scalar product ${ }^{6}$,

$$
\Delta \sigma_{C D}=\alpha_{s} \xi(\boldsymbol{k} \cdot \boldsymbol{A})
$$

where the dynamical factor $\alpha_{s}$ is a true scalar (that may be represented, e.g., as the real part of a product of particular components of the photoprocess amplitudes). Therefore, the existence of $\mathrm{CD}$ for the cases considered above originates from an intrinsic 'chiral' vector of the problem, $\boldsymbol{A}$, that balances the time-oddness and the pseudoscalar property of the 'CD vector' $\xi \boldsymbol{k}$.

Dichroic effects in photoprocesses with unpolarized targets have a physical origin that is different from those mentioned above since in this case the initial atomic system does not involve any chiral vectors $\boldsymbol{A}$. As a general analysis shows [11, 12], these effects originate instead from the interference between real and imaginary (non-Hermitian or 'dissipative') parts of the quantum transition amplitudes (or, alternatively, of the nonlinear susceptibilities [13]). From a general consideration of the simplest possible form of the CD term (i.e. similar to equation (1) for polarized targets), it is clear that in many photoprocesses involving free atoms it is possible to construct a time-even pseudovector as the vector product $\left[\boldsymbol{a}_{1} \times \boldsymbol{a}_{2}\right]$ of vectors of the problem having the same temporal symmetry. Obviously these vectors $\boldsymbol{a}_{i}$ are different in different processes and their choice depends on the concrete problem studied (e.g. there are the momenta of incident and scattered electrons in a bremsstrahlung process or the momenta

\footnotetext{
6 When there exist other vectors in the problem, in addition to $\boldsymbol{A}$, additional CD terms are possible. For example, for the case of photoionization, there appears (in addition to (1)) a CD term with the form $\alpha_{s}^{\prime} \xi(\boldsymbol{k} \cdot \boldsymbol{p})(\boldsymbol{A} \cdot \boldsymbol{p})$, where $\boldsymbol{p}$ is the photoelectron momentum and $\alpha_{s}^{\prime}$ is a true scalar coefficient [8].
} 
of the two escaping electrons in double photoionization of a target atom). However, the timeodd dynamical (scalar) parameter, $\beta_{s}$ (that now replaces the time-even $\alpha_{s}$ in (1)), can arise only as the result of the interference between real and imaginary parts of some particular components, $f_{i}$, of the transition amplitudes, i.e. it may be represented $\operatorname{as}^{7} \beta_{s} \sim \operatorname{Im}\left\{f_{i} f_{j}^{*}\right\}$. Dichroic effects thus provide the possibility for direct experimental measurements of this interference. One important difference between dichroic effects for polarized and unpolarized targets is that for the former these effects exist in the total cross sections as well (as is clear from (1), where $\boldsymbol{A}$ is a fixed vector), whereas the 'chiral' properties of unpolarized targets arise from the geometry of the particular experimental measurement, i.e. they depend on the directions of the vectors $\boldsymbol{a}_{i}$. Thus dichroic effects in processes involving unpolarized targets are manifested mainly in ADs, and in most cases they vanish in angle-integrated, total cross sections.

CD in photoprocesses involving unpolarized atomic targets was first discussed in 1992 [15] for the case of single-photon, double photoionization of helium. The vector product, $\left[p_{1} \times p_{2}\right]$, of the momenta of the escaping electrons is a time-even pseudovector and the time-odd scalar parameter, $\beta_{s}$, involves the non-Hermitian part of the transition amplitude. Thus the CD term in the double photoionization cross section is given by an expression similar to (1), but with the substitution of the time-even pseudovector $\left[\boldsymbol{p}_{1} \times \boldsymbol{p}_{2}\right]$ for $\boldsymbol{A}$ and of the time-odd dynamical factor $\beta_{s}$ for $\alpha_{s}$ [12]. (This process has attracted much attention recently as it provides a very sensitive test of the importance of electron correlations; see reviews [16, 17] on the current status of this problem.) Somewhat later [14], CD in light scattering by unpolarized atoms was predicted. Here the wavevectors of the incident $\left(\boldsymbol{k}_{1}\right)$ and scattered $\left(\boldsymbol{k}_{2}\right)$ photons form a time-even pseudovector, $\boldsymbol{k}_{1} \times \boldsymbol{k}_{2}$, and thus the CD for this case occurs only by going beyond the electric dipole approximation. It leads, in particular, to the production of a circularly polarized component in the scattered light resulting from the scattering of linearly polarized optical radiation by, e.g., a ground state alkali atom. The $\mathrm{CD}$ effects in the scattering of $x$ - and $\gamma$-rays by heavy atoms and in other bound-bound relativistic two-photon transitions (that also originate from non-dipole processes) have been discussed in [18] and [19].

Besides double photoionization and photon scattering, significant $\mathrm{CD}$ effects are possible in other atomic processes. In laser-assisted electron-atom collisions Fainshtein et al [20] provide perturbative treatments of CD arising from the linear-in-laser-intensity correction to the Rutherford formula for elastic $\mathrm{e}-\mathrm{H}^{+}$scattering in the presence of an (elliptically polarized) laser field. Recent calculations of $\mathrm{CD}$ for $\mathrm{e}-\mathrm{H}^{+}$scattering in a strong circularly polarized field have been performed in [21]. For bremsstrahlung processes, spontaneous one-photon emission has been treated in [12] and stimulated one-photon emission has been treated in [22]. In scattering of charged particles from oriented atomic targets (produced, e.g., via optical pumping), the CD effect leads to the dependence of cross sections on the helicity of the laser pump beam [23]. This effect has been observed recently for scattering into the capture channel [24] as well as in cross sections of e-2e impact ionization of atoms assisted by a circularly polarized laser field resonant with an atomic transition $[25,26]$. New aspects of the CD effect appear in the presence of a static electric field (in addition to a laser field), which induces an anisotropy in an initially isotropic atomic medium. At present such anisotropy-induced CD was investigated in two-photon transitions between atomic levels with opposite parities (with a DC-field-induced resonance) [27], in the total photoelectron yield from a combined two-photon plus second harmonic (one-photon) ionization of alkali atoms [28], in nonresonant dipole-allowed light scattering [29] and in (dipole-forbidden) resonant three-photon scattering by ground state atoms [30].

7 In particular, the width $\Gamma_{r}$ of an intermediate resonant level may serve as the parameter $\beta_{s}$ [14]. 
It is not so widely known that in multiphoton processes involving two or more identical laser photons, a new type of dichroic effect is possible that vanishes for the case of completely circular or linear polarization and that exists only for 'intermediate' ellipticity, $0<|\xi|<1$. As was discussed by Manakov [11], these effects have the same interference origin as CD effects. To distinguish them from $\mathrm{CD}$, which is maximum at $100 \%$ circular polarization, the term ED was introduced $[13,31]$ for those polarization effects that are proportional to the product, $l \xi$, of the linear $(l)$ and circular $(\xi)$ polarization degrees of a laser beam. In principle, under suitable conditions (e.g. non-Hermiticity of a transition amplitude, a specialized geometry of fields, etc), a more or less considerable ED effect is possible in a majority of single-colour and multicolour multiphoton processes. Note that in the multicolour case the CD effect (which is non-vanishing only for $\xi= \pm 1$ ) may be possible in parallel with the ED effect, although the two effects are described by different sets of atomic parameters and may be measured independently, thereby providing different information on the atomic states involved. Such a situation has been analysed for three-photon (dipole-allowed) transitions between bound atomic states [31]; it may also be realized in the ionization of atoms in the presence of a strong laser field and one of its higher harmonics [32] (see also [33]), as well as in standard two-colour frequency mixing in gas samples [34].

Measurements of light helicity-dependent interference effects, such as ED, provide effective tools for polarization control, on the one hand, and for distinguishing between different theoretical models, on the other hand, in fundamental intense laser-atom processes, such as, e.g., ionization, HHG and laser-assisted collisions. Note that in the latter process the ED effect is possible only when there is an exchange of two or more photons (e.g. as in stimulated emission or absorption) while in elastic scattering and single-photon scattering only the CD effect takes place. All dichroism effects vanish in the Born (or the Born-Volkov) approximation, when only the momentum transfer, $\boldsymbol{p}_{i}-\boldsymbol{p}_{f}$, of a projectile enters the result for the cross section [20, 22]. (For this reason the CD effect was not obtained in a recent analysis [35] of e-H scattering in a strong circularly polarized field; it appears only in the presence of an additional, linearly polarized laser beam [36].) In single-colour HHG the ED effect is observable only in measurements that determine the polarization state of the harmonics (e.g. by measuring the intensity of the linearly polarized component of the $n$th harmonic produced by an elliptically polarized pump field [13]). In contrast, the presence of a static or low-intensity, low frequency laser field in addition to a strong fundamental laser beam results in a significant ED effect in the total harmonic yield [37]. Other recent results on polarization effects in HHG can be found in a review [38].

The first analysis of the ionization of atoms by an elliptically polarized field was performed in 1966 [2] (and has been reviewed recently in [39] with an extension to the case of over-barrier ionization). In this analysis the well-known Keldysh results [1] for linear and circular laser polarizations were generalized for the case of a general elliptic polarization. The ellipticityinduced distortion of ADs in the tunnelling regime (by an intense, low frequency field) has been analysed in more detail in [40,41]. These latter calculations demonstrate considerable ellipticity effects in certain situations (in particular, the stretching of ADs along the minor axis of the polarization ellipse of a laser field). The first experimental measurement of atomic ionization by a strong elliptically polarized field was performed in 1988 by Bashkansky et al [42]. Recently, the significant ellipticity dependence of ADs for individual ATI electron peaks was measured for both low- [43] and high-energy [44] parts of above-threshold photoelectron spectra. These effects are explained by the interference of tunnelling electron trajectories, taking into account rescattering effects $[43-46]^{8}$.

8 Although the interference exists also for linear polarization, it is most clearly exhibited for nonzero ellipticity. 
The sensitivity of the ADs to the sign of the ellipticity, i.e. the ED effect, was observed first in the experiment of Bashkansky et al [42] on the multiphoton ionization of rare gases by an elliptically polarized field: the observed reduction of the symmetry of the ADs in the plane of the elliptic laser polarization (as compared to the cases of linear and circular polarizations) is the result of nonzero ED terms in the angular distribution cross sections. This asymmetry has attracted much interest because it cannot be explained within the framework of the approximate Keldysh-like theories (e.g. such as the strong field approximation (SFA)) and requires a more detailed account of the binding potential. Indeed, such more detailed analyses $[47,48]$ predict the observed two-fold symmetry of the ADs (instead of a four-fold one, as in the case of linear polarization). The general treatment of the ED effect in three-dimensional photoelectron ADs for two-photon ionization of atoms and rather extensive numerical results for the hydrogen $|n l\rangle$ states with $n \leqslant 10$ can be found in [49] (see also [32, 50] on dichroic effects in twocolour, two- and three-photon ionization processes). The analysis of the AD asymmetry in the SFA with inclusion of Coulomb effects is presented in [51]. The entire three-dimensional $\mathrm{AD}$ in two-photon ionization by an elliptically polarized field has been measured first in a recent experiment [52] for the rubidium atom. These results exhibit a clear ED asymmetry. Although this experiment was performed for a fixed sign of the laser ellipticity and the authors do not discuss the ED effect as such, they emphasize the efficiency of measurements using an elliptically polarized field for the extraction of information on the radial ionization amplitudes and scattering phases.

As shown by the above brief review, the use of laser fields with an elliptical polarization adds a new dimension to the analysis of multiphoton interactions. The study of ellipticity(and, especially, helicity-) dependent effects provides new information on atomic processes that is inaccessible in measurements employing purely linear or circular polarizations. In addition, the high sensitivity of the dichroic parameters to the interaction of a bound electron with the laser field and to the binding potential provides a sensitive means (because it depends on quantum interference phenomena) for distinguishing between different theoretical models in strong laser-atom physics.

\subsection{Status of multiphoton detachment of negative ions}

The number of existing experimental results on multiphoton detachment of negative ions is significantly fewer than that for atoms, even though the first experimental measurements (for two-photon detachment of the $\mathrm{I}^{-}$ion) were performed in 1965 [53], simultaneously with the first observations of multiphoton ionization of atomic and molecular samples [54]. Experimental interest in negative ions burgeoned only at the end of the 1980s, when a number of measurements (mostly, for negative halide ions) were performed in the perturbative laser intensity regime: in 1987 Trainham et al [55] measured the two-photon detachment cross section for $\mathrm{Cl}^{-}$, and beginning in 1989, a number of other groups carried out measurements of two- and three-photon cross sections for other ions [56-60]. In 1990, Blondel et al [61] measured the first multiphoton detachment ADs (for two- and three-photon detachment of $\mathrm{Br}^{-}$). Subsequently, they presented extensive results for all negative halide ions [62]. In 1991, the first measurements of above-threshold (excess-photon) detachment (ATD) with observation of about two excess photons were performed for $\mathrm{F}^{-}$[63], $\mathrm{Au}^{-}$[64] and $\mathrm{Cl}^{-}$[65] using $\mathrm{Nd}$ :YAG lasers with intensities greater than $10^{12} \mathrm{~W} \mathrm{~cm}^{-2}$.

The observation of a multiphoton regime in laser detachment of $\mathrm{H}^{-}$requires the use of longer wavelength laser sources than the Nd:YAG laser. The first observations were reported in [59] and studied in more detail in [66] for photon energies from 0.15 to $0.39 \mathrm{eV}$ and laser intensities from 2 to $12 \mathrm{GW} \mathrm{cm}^{-2}$. In particular, characteristic threshold structures and intensity-dependent (ponderomotive) shifts of the detachment threshold energy were observed. 
An absorption of excess photons in ATD of $\mathrm{H}^{-}$with $\mathrm{Nd}$ :YAG light was observed by Zhao et al [67]: two-photon absorption was measured (with little evidence of three-photon absorption) on top of the background of the open one-photon detachment channel. In more recent experiments $[68,69]$, the two-photon detachment of $\mathrm{H}^{-}$was measured near the threshold of the one-photon channel (where the latter is suppressed in view of the Wigner threshold law [70] (see (B.25) below). However, evidence of ATD spectra similar in clarity to ATI spectra typical for atoms at low intensities have been demonstrated only in a very recent experiment [71] in which at least three ATD channels for $\mathrm{H}^{-}$have been observed using a short infrared pulse of $2.15 \mu \mathrm{m}$ wavelength (for which the lowest detachment channel is the two-photon one).

All of the experimental studies discussed above were performed using linearly polarized light. At first sight, this case seems to be the most interesting one since, e.g., for the case of circularly polarized light, the interference of intermediate and final channels having different angular momenta $l$ for the escaping electron vanishes owing to dipole selection rules; the angular momentum of the detached electron after absorption of $n$ circularly polarized photons is $l=n$ (for an initial s-electron). Thus, the detachment cross sections are less sensitive to details of the binding potential. For low photoelectron energies, this fact is especially important for negative ions because the scattering phases, $\delta_{l}$, for a short-range potential decrease sharply with increasing $l$ and, in view of the Wigner threshold law, multiphoton cross sections for the case of circular polarization may be expected to be suppressed. However, as was discussed in section 1.1, the use of an elliptical polarization allows one to obtain new information on the atomic binding potential which in principle cannot be extracted from experiments with purely linear (or purely circular) polarization. The $\mathrm{CD}$ effect in multiphoton detachment was observed by Sturrus et al [72] in two-colour, two-photon detachment of $\mathrm{Cl}^{-}$using a combination of two counterpropagating laser pulses (in the near-infrared (Nd:YAG) and in the VUV) having either the same or opposite circular polarizations. The relative difference of the two cross sections for the two measurements (i.e. changing $\xi_{i r}=+1$ to -1 for the infrared photon) is $8.5 \%$, with high accuracy. Obviously, this CD effect is similar to that for chiral systems, since the vector $\xi_{v u v} \boldsymbol{k}_{v u v}$ stands here for the chiral vector $\boldsymbol{A}$ in equation (1). Blondel and Delsart [73] performed the first measurements with elliptically polarized light (using the second harmonic of the Nd:YAG laser to analyse the ADs for two-photon detachment of $\mathrm{I}^{-}$and $\mathrm{F}^{-}$) and emphasized its necessity for performing a 'complete' multiphoton detachment experiment in the perturbative regime [74]. A general perturbative analysis of ellipticity effects and in particular the ED asymmetry in the two-dimensional AD (namely, in the plane orthogonal to the direction of the laser beam) for the case of $n$-photon detachment of negative halide ions was given in [75]. Extensive experimental data for the orthogonal plane geometry have been presented in [76] for three- and four-photon detachment of $\mathrm{I}^{-}, \mathrm{Cl}^{-}$and $\mathrm{F}^{-}$by a Nd:YAG laser (although the measured ED asymmetry is not so significant for halide ions). Reference [77] presents perturbative calculations of the ED asymmetry in the photoelectron ADs for two- and three-photon, above-threshold detachment of the $\mathrm{H}^{-}$ion. These calculations have been performed for an orthogonal plane geometry. They demonstrate a significant variation of the degree of asymmetry with respect to both the ellipticity parameter and the laser frequency. Recently, a nonperturbative analysis of ellipticity-induced distortion of ADs and ED parameters together with numerical results for ATD of $\mathrm{H}^{-}$(modelled by a zero-range potential) have been presented for $n=2-5$ in [78]. Significant modifications of the three-dimensional ADs as well as sharp variations of the ED parameters near the ATD thresholds were predicted [78]. However, at the present time no experiments for multiphoton detachment of $\mathrm{H}^{-}$using elliptically polarized photons have yet been performed.

Regarding the various theoretical approaches and numerical methods for calculation of multiphoton detachment rates and ADs, we mention first that, beginning from the pioneering 
calculation of two-photon detachment from negative ions in 1967 [79], simple, short-range potential models were used in many studies of two- and three-photon detachment of $\mathrm{H}^{-}$(see, e.g., [80-86]), and also for $n>3[85,86]$. In these works a 'reduced' $\delta$-model potential was used, in which the scattering phase in the s-wave part of the continuum wavefunctions was neglected. It was quickly noted that neglect of continuum phase shifts, particularly the swave phase shift, was a significant source of error, particularly for the $n=2$ detachment cross section [87, 88]. Meanwhile, since the mid-1980s there have been an increasing number of more elaborate theoretical treatments of multiphoton detachment for both $\mathrm{H}^{-}$and heavier negative ions that include, to varying degrees, the effects of electron correlations, both perturbatively and nonperturbatively [87-121]. Nevertheless, at least for the well-studied $\mathrm{H}^{-}$ negative ion, a general conclusion is that use of the zero-range potential model, including at least the s-wave continuum phase shifts, is sufficient to obtain quantitative agreement with results of the most elaborate theoretical approaches [87, 88, 105, 117]. At present, for accurate predictions of multiphoton detachment cross sections different numerically intensive $a b$ initio methods are used, such as the $R$-matrix Floquet approach [108, 109, 112, 116, 118], various forms of $B$-spline methods [77, 105, 107, 113] (these methods have been reviewed recently in [120]) and the direct solution of the time-dependent Schrödinger equation for laser pulse fields [119]. The most detailed results for partial $n$-photon detachment rates of $\mathrm{H}^{-}$, including results for both weak and strong fields, have been obtained recently by Nicolaides et al $[122,123]$ using a many-electron version of the Floquet approach. Nonperturbative results for $\mathrm{ADs}$ in multiphoton detachment of $\mathrm{H}^{-}$for three laser wavelengths, $\lambda=10.6,1.908$ and $1.064 \mu \mathrm{m}$ can be found in [124-126]. (These authors used the Floquet approach and a pseudospectral method for the discretization of the Floquet Hamiltonian, in conjunction with a parametrized one-electron model potential [102].)

Despite the many theoretical studies for multiphoton detachment, only the simplest cases of linear and/or circular polarizations of the laser field have been examined in the many papers cited above (with the exception of the perturbative treatment of [77] for $\left.\mathrm{H}^{-}\right)^{9}$. The generalization of numerically intensive methods to the case of an arbitrary (elliptical) polarization is not straightforward and leads to much more tedious calculations owing to the larger number of dimensions that must be treated and to the consequent need for extended sets of basis functions, inclusion of higher angular momentum states, etc. Furthermore, in practice, direct numerical analysis may be performed, of course, only for limited sets of field parameters (i.e. frequency, intensity and ellipticity). For these reasons, it would be useful to have a general understanding of the global dependence of multiphoton detachment ADs on these parameters based on simple analytical models. One possibility is to use the Keldysh approach results [1], which are most appropriate for weakly bound systems (although only for small frequencies, $\hbar \omega \ll\left|E_{0}\right|$, where $\left|E_{0}\right|$ is the binding energy). Indeed, following [1], Gribakin and Kuchiev [128] performed a broad and detailed analysis of multiphoton rates and ADs within the adiabatic approach (for its generalization to bichromatic fields, see [129]). Their predictions are in reasonable agreement with experimental results for two- and threephoton detachment from negative halide (and some other) ions (see $[128,130]$ ) and also in good agreement with a recent experiment for $\mathrm{H}^{-}$[71]. However, this development of the Keldysh approach was performed only for the case of linear polarization. Furthermore, for a strong field its accuracy is unclear since, in general, the adiabatic approach is valid only for weak (although nonperturbative) fields, in which the laser field amplitude $F$ is small compared to the typical 'internal field', $F_{0} \sim \sqrt{m\left|E_{0}\right|^{3}} /|e| \hbar$.

9 For a $\delta$-model potential, the analytical perturbative analysis of ellipticity effects was performed for one- and twophoton partial rates $[81,127]$ and for the two-photon AD including the ED effect [49]. 
Another possibility to reveal the general features of multiphoton detachment in a strong laser field is to model the short-range potential of the outer (weakly bound) electron of a negative ion by a zero-range $(\delta$-model) potential. The $\delta$-model potential allows one to obtain an exact solution for the case of a strong laser field having an arbitrary (elliptical) polarization. The results of this model may be presented in a simple analytical form that is convenient for a detailed analysis of the frequency, polarization and laser intensity dependences of multiphoton ADs, including for the case of detachment with absorption of excess photons. In particular, such results may provide a theoretical justification for the extension of existing experiments on multiphoton detachment for linearly polarized laser fields to the case of elliptical polarization. Despite its simplicity, the $\delta$-model potential gives a reasonably accurate description of photodetachment of negative ions having a ground state outer electron in an sstate, such as, e.g., $\mathrm{H}^{-}$(for photoelectron energies below the $\mathrm{H}(n=2)$ excitation threshold). Moreover, this model potential has already been used successfully to analyse a number of important questions in strong field theories of laser-atom interactions. As examples, we note that, based upon this model, the first nonperturbative calculation employing the complex quasienergy (or non-Hermitian Floquet) approach was performed [131]; the initial results of the adiabatic Keldysh approach [1,2] were shown to be limiting cases of ab initio results for the imaginary part of the complex quasienergy for this model in a strong elliptically polarized field [127]; the existence of a 'plateau' in HHG and ATI spectra was confirmed by quantitative results for the $\delta$-model potential $[132,133]$ and, very recently, the exact solution for this model has been used in the analysis of the quasistationary stabilization (QS) problem for a short-range potential [134], for the quantum interpretation of resonance-like structures experimentally observed in high energy ATI [135] and HHG [136] spectra and for the prediction of plateau effects in laser-assisted electron-atom scattering [137].

\subsection{Outline of this review}

In this review we formulate a general approach for the analysis of ADs in a strong elliptically polarized field and present both perturbative and nonperturbative treatments of ED effects in three-dimensional ADs for the case of $n$-photon detachment of an electron bound in a short-range $(\delta$-model) potential. The main advantage of this treatment is that, for this model problem, an accurate ( $a$ b initio) quasistationary quasienergy states (QQES) formulation for a strong laser field may be formulated. It permits simple analytical results for the perturbative regime and exact numerical results for the strong field limit which together allow one to analyse various qualitative aspects of the frequency and polarization dependences of multiphoton cross sections for a weakly bound electron. Besides analysing general features of the ADs of detached electrons produced by a laser field having photon energy $\hbar \omega$ smaller than the (unperturbed) binding energy, $\left|E_{0}\right|$, the second question we address in this review is the intensity and polarization dependence of both total and differential rates of photodetachment in the high frequency limit, $\hbar \omega>\left|E_{0}\right|$, when the ordinary photoeffect takes place at low intensities. This question is closely related to the widely discussed problem of adiabatic (or quasistationary) stabilization of atomic decay rates in the presence of a strong high frequency field. In contrast to the atomic case, where typical laser frequencies (with $\hbar \omega \sim 1 \mathrm{eV}$ ) are much less than ionization potentials, i.e. they correspond to the low frequency limit, $\hbar \omega \ll\left|E_{0}\right|$, for negative ions they are of the order of (or even exceed) $\left|E_{0}\right|$, as for $\mathrm{H}^{-}$, where $\left|E_{0}\right|=0.7542 \mathrm{eV}$. For this reason, our analysis here focuses on perturbative and strong field results for $n$-photon detachment with $n<10$, including the high frequency domain. We do not analyse here the strong field, low frequency limit (in which, for neutral targets, the high energy plateau in the ATI spectrum occurs), because for negative ions this regime is not of current experimental interest. 
This review is organized as follows. In section 2 we review the theory upon which our analysis of multiphoton detachment is based. We first present our notations and the basic definitions of the QQES approach. We then survey our prior QQES results for an electron in a three-dimensional $\delta$-model potential subjected to a strong monochromatic laser field having an elliptical polarization. The basic equations for the QQES wavefunction, $\Phi_{\epsilon}(\boldsymbol{r}, t)$, and for the complex quasienergy, $\epsilon$, corresponding to an initial bound state electron having the energy $E_{0}$ are presented in section 2.3. These results are necessary for an accurate derivation of the general equations (presented in section 2.4) for the differential rates of multiphoton detachment, taking exact account of both strong laser field and binding potential effects. Finally, in sections 2.5 and 2.6 we perform an analytical analysis of two important limiting cases:

(i) in section 2.5 we show how both the wavefunctions and the differential rates of the Keldysh approach follow as limiting cases of our exact QQES results;

(ii) in section 2.6 we present perturbative (in the laser intensity, $I$ ) expansions for the $n$-photon detachment amplitude and the corresponding differential rates.

Both Rayleigh-Schrödinger (RS) and Brillouin-Wigner (BW) expansions are analysed. The latter involves the exact complex quasienergy $\epsilon$ and thus is applicable for higher intensities, far beyond the radius of convergence for the RS perturbation theory (PT) results. We present analytical results for the differential rates of $n$-photon detachment in the lowest order of PT (LOPT), including explicit results for $n=2-5$ and a short discussion of the BW expansion for $n=2$. Also, threshold effects in the PT regime are discussed briefly.

Sections 3-6 present detailed analyses of laser ellipticity and ED effects for multiphoton ADs and decay rates in various intervals of laser frequencies and intensities. In section 3 the LOPT results of section 2.6 are analysed in more detail: the general analysis of $n$-photon, three-dimensional ADs for an elliptical polarization is presented (section 3.1), the magnitude and general properties of ED effects in ADs are discussed (section 3.2) and the good agreement of our numerical results with results of both recent experiments and other calculations for $\mathrm{H}^{-}$ is demonstrated. In section 4 , after a brief discussion of general symmetry properties of ADs in a strong elliptically polarized field, we present the results of nonperturbative, strong field calculations for both partial ( $n$-photon) and total (summed over $n$ ) ADs, including results for the frequency and intensity dependences of ED parameters. New features of the ADs and the ED effect specific to the nonperturbative regime are discussed.

In section 5, photodetachment in a high frequency field, $\hbar \omega>\left|E_{0}\right|$, is analysed. Its dependence on $\omega$ and on the laser polarization is discussed for a wide interval of intensities, from the LOPT regime up to the strong field limit. More specifically, section 5.1 presents analytical results for the electron $\mathrm{AD}$ resulting from one-photon detachment, taking into account intensity corrections of orders $I$ and $I^{2}$ (in both RS and BW versions of PT) together with a discussion of the onset of stabilization-like behaviour. Numerical results for stronger fields, where above-threshold, $n$-photon channels contribute and the QS regime is realized, are discussed in sections 5.2 and 5.3. Finally, in section 6 we present analytical estimates and numerical results for the total decay rate in superstrong fields, when several lowestorder detachment channels are closed; we demonstrate that (for any frequency) the results become similar to those for decay in a strong static electric field. In section 7, we present our conclusions.

A number of mathematical details of the QQES theory, upon which our analyses are based, are presented in the appendices for interested readers. In appendix A we discuss the normalization of the QQES wavefunction $\Phi_{\epsilon}(r, t)$, which is divergent for $r \rightarrow \infty$. In appendix B we give a detailed analysis of the Fourier coefficients of $\Phi_{\epsilon}(r, t)$ at the origin 
(i.e. $r \rightarrow 0$ ). In appendix $\mathrm{C}$ we derive the $n$-photon detachment amplitude from the exact QQES wavefunction.

Note that the Fourier coefficients analysed in appendix B are key elements of the QQES approach for a $\delta$-model potential, and in particular for the ADs and partial rates of $n$-photon detachment. They contain complete information on the points of non-analyticity of the detachment amplitude as a function of the laser field amplitude and frequency. Consequently, they play an essential role for a correct description and proper physical interpretation of polarization and threshold effects in ADs for multiphoton detachment. In appendices B.1 and B.2 we present both BW and RS perturbative expansions (in the laser intensity) of the exact results for the normalization factor, for the Fourier coefficients of the QQES wavefunction at the origin and for the quasienergy $\epsilon$, all of which are necessary ingredients for accurate $\mathrm{BW}$ and RS PT analyses of ADs. The details of numerical calculations in the strong field regime, characteristic plateau features in the strong field behaviour of the Fourier coefficients and their strong field 'rescattering approximations' are discussed in appendices B.3 and B.4.

\section{Basic results of the QQES approach for the $\delta$-model potential and general equations for multiphoton detachment rates}

\subsection{Definitions and scaled units}

We use the velocity gauge for the dipole interaction of an electron in the atomic potential $U(\boldsymbol{r})$ with a monochromatic laser field (though all our final results are gauge-invariant):

$$
V(\boldsymbol{r}, t)=\frac{|e|}{m c} \hat{\boldsymbol{p}} \cdot \boldsymbol{A}(t)+\frac{e^{2}}{2 m c^{2}} A^{2}(t),
$$

where

$$
\boldsymbol{F}(\omega t)=-\frac{1}{c} \frac{\partial}{\partial t} \boldsymbol{A}(t)=F \operatorname{Re}\left(e \mathrm{e}^{-\mathrm{i} \omega t}\right),
$$

is the electric vector and $e$ is the unit (complex) polarization vector, $e \cdot e^{*}=1$. For the general case of an elliptic polarization, we use the following invariant parametrization of $e$ :

$$
e=\frac{\hat{\epsilon}+i \eta[\hat{k} \times \hat{\epsilon}]}{\sqrt{1+\eta^{2}}}, \quad-1 \leqslant \eta \leqslant 1
$$

where $\eta$ is the ellipticity and $\hat{\epsilon}$ and $\hat{k}$ are the unit vectors in the directions of the major axis of the polarization ellipse and the wavevector of the laser field, $k$, respectively. Instead of the ellipticity, for the description of the polarization state of a laser it is often convenient to use the degrees of linear $(l)$ and circular $(\xi)$ polarizations (see, e.g., [138])

$$
l=e \cdot e=\frac{1-\eta^{2}}{1+\eta^{2}}, \quad \xi=\mathrm{i}\left(\hat{k} \cdot\left[e \times e^{*}\right]\right)=\frac{2 \eta}{1+\eta^{2}},
$$

which appear naturally in the theory (see below). Note that $l^{2}+\xi^{2}=1$ for a completely polarized laser field. For the analysis of ADs it is convenient to use the geometry presented in figure 1, where $\alpha$ is the angle between the unit vector $n$ in the direction of the escaping electron and $\hat{\epsilon}$ (the major axis of the polarization ellipse) and $\beta$ is the angle between $n$ and $[\hat{k} \times \hat{\epsilon}]$ (the minor axis). $\theta$ is the polar angle of the vector $n$ in the coordinate frame having the $z$ axis along $\hat{k}$ and the $x$ axis along $\hat{\epsilon}$. (The corresponding azimuthal angle of the vector $n$ is $\varphi$.) In the chosen geometry we have

$$
\begin{aligned}
& |e \cdot n|^{2}=\frac{1}{2} \sin ^{2} \theta(1+l \cos 2 \varphi)=\frac{1}{4} \sin ^{2} \theta\left|\sqrt{1+\xi} \mathrm{e}^{\mathrm{i} \varphi}+\sqrt{1-\xi} \mathrm{e}^{-\mathrm{i} \varphi}\right|^{2}, \\
& (\boldsymbol{e} \cdot \boldsymbol{n})^{2}=\frac{1}{2} \sin ^{2} \theta(l+\cos 2 \varphi+\mathrm{i} \xi \sin 2 \varphi)=\frac{1}{4} \sin ^{2} \theta\left(\sqrt{1+\xi} \mathrm{e}^{\mathrm{i} \varphi}+\sqrt{1-\xi} \mathrm{e}^{-\mathrm{i} \varphi}\right)^{2} .
\end{aligned}
$$




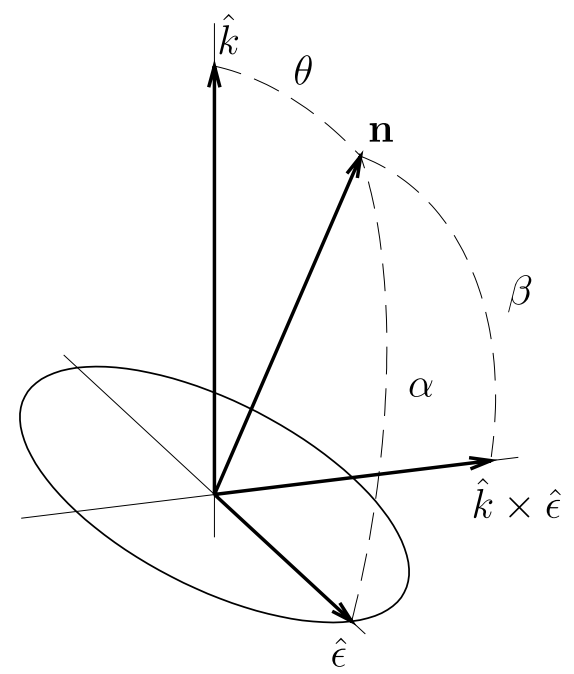

Figure 1. Geometry of a laser field (propagating along $\hat{k}$ and having the major axis of its polarization ellipse along $\hat{\epsilon}$ ) and the momentum direction, $\boldsymbol{n}$, of a detached electron.

To describe a weakly bound electron we employ the $\delta$-model potential [139]:

$$
U(r)=\frac{2 \pi \hbar^{2}}{m \kappa} \delta(r) \frac{\partial}{\partial r} r,
$$

with the binding energy $E_{0}=-\hbar^{2} \kappa^{2} / 2 m$, and the bound-state wavefunction

$$
\psi_{0}(r)=N \frac{\mathrm{e}^{-\kappa r}}{r},
$$

where $N=\sqrt{\kappa / 2 \pi}$. In order to present our results in the most general form, it is convenient to use scaled units based on the single parameter $\kappa$ of the model potential (8): the length unit is $1 / \kappa$; the energy and the frequency are measured in units of $\left|E_{0}\right|$ and $\left|E_{0}\right| / \hbar$; the field amplitude $F$ is measured in units of the 'internal field', $F_{0}=\sqrt{2 m\left|E_{0}\right|^{3}} /|e| \hbar$ and the corresponding scaled unit of the intensity, $I=c F^{2} / 8 \pi$, is $I_{0}=c F_{0}^{2} / 8 \pi$. As an example, for $H^{-}(\kappa=0.2356 \mathrm{au})$ we have $F_{0}^{H^{-}}=3.362 \times 10^{7} \mathrm{~V} \mathrm{~cm}^{-1}$ and $I_{0}^{H^{-}}=1.498 \times 10^{12} \mathrm{~W} \mathrm{~cm}^{-2}$. Thus, in scaled units, we have $I=F^{2}$. The cross section, $\sigma$, in our units $\left(\kappa^{-2}\right)$ is connected with that in atomic units (au), $\sigma^{(a u)}$, by the relation: $\sigma^{(a u)}=\sigma\left(E_{a u} / 2\left|E_{0}\right|\right)$, where $E_{a u}=m e^{4} / \hbar^{2}$.

We employ in this paper the standard normalization constant $N=\sqrt{\kappa / 2 \pi}$ for the initial bound state (9), which is self-consistent for the (one-parameter) $\delta$-potential model. However, it is well known that for real negative ions more exact results may be obtained using, instead of $N$, a corrected normalization constant, $N_{c}$, which may be obtained, e.g., by analysing the asymptotic behaviour of the wavefunction for large $r$ (cf $[29,128,140]$ for details). For this case, our results for the photodetachment cross sections, $\sigma^{(n)}$, and/or probabilities should be multiplied by the renormalization factor $A_{c}=2 \pi N_{c}^{2} / \kappa$. For $\mathrm{H}^{-}$the factor $A_{c}$ has the value 2.6551. Thus, e.g.,

$$
\sigma^{(n), \mathrm{H}^{-}}=A_{c} \sigma^{(n)} \text {. }
$$

\subsection{Background results of the QQES approach for a strong laser field}

The simplest way to analyse the laser-field-induced exponential (in time) decay of a bound level in the atomic potential $U(r)$ is by use of the QQES approach (see, e.g., [141]), which is 
similar to the well-known quasistationary (or resonance) states approach for radiationless atomic problems with time-independent Hamiltonians. In calculations of the decay rate (e.g. ionization or detachment) of a quantum system subjected to a (periodic in time) strong external perturbation, this method allows one to reduce the direct solution of the initial value (Cauchy) problem for the time-dependent Schrödinger equation to a much simpler eigenvalue problem for the complex quasienergy, $\epsilon$. In the QQES approach the wavefunction of an initial bound state, $\psi_{n}(\boldsymbol{r}) \exp \left(\mathrm{i} E_{n} t\right)$, in the presence of an (adiabatically turned on) strong laser field (3) has the quasienergy form

$$
\Psi_{n}(\boldsymbol{r}, t)=\mathrm{e}^{-\mathrm{i} \epsilon_{n} t} \Phi_{\epsilon_{n}}(\boldsymbol{r}, t),
$$

with the periodic QQES wavefunction, $\Phi_{\epsilon_{n}}(\boldsymbol{r}, t)=\Phi_{\epsilon_{n}}(\boldsymbol{r}, t+2 \pi / \omega)$, and the complex quasienergy

$$
\epsilon_{n}=\operatorname{Re} \epsilon_{n}-\mathrm{i} \frac{\Gamma_{n}}{2} .
$$

The quantities $\operatorname{Re} \epsilon_{n}-E_{n}$ and $\Gamma_{n}$ determine the Stark shift and the total decay rate of an initial bound state with the energy $E_{n} . \epsilon_{n}$ and $\Phi_{\epsilon_{n}}(r, t)$ satisfy the 'stationary' Schrödinger equation, $\mathcal{H} \Phi_{\epsilon_{n}}=\epsilon_{n} \Phi_{\epsilon_{n}}$, with the Hamiltonian, $\mathcal{H}=-\nabla^{2}+U(\boldsymbol{r})+V(\boldsymbol{r}, t)-\mathrm{i} \partial / \partial t$ and with the complex boundary condition at $r \rightarrow \infty$ (cf [142]).

For a strong laser field, various forms of eigenvalue equations for the complex quasienergy may be used [143]. In particular, $\epsilon$ and $\Phi_{\epsilon}(\boldsymbol{r}, t)$ may be obtained as the solution of the integral eigenvalue problem

$$
\Phi_{\epsilon}(\boldsymbol{r}, t)=\int \mathrm{d} t^{\prime} \int \mathrm{d} \boldsymbol{r}^{\prime} \mathrm{e}^{\mathrm{i} \epsilon\left(t-t^{\prime}\right)} G^{(+)}\left(\boldsymbol{r}, t, \boldsymbol{r}^{\prime}, t^{\prime}\right) U\left(\boldsymbol{r}^{\prime}\right) \Phi_{\epsilon}\left(\boldsymbol{r}^{\prime}, t^{\prime}\right),
$$

where $G^{(+)}\left(\boldsymbol{r}, t, \boldsymbol{r}^{\prime}, t^{\prime}\right)$ (or $G^{(-)}\left(\boldsymbol{r}, t, \boldsymbol{r}^{\prime}, t^{\prime}\right)$ ) is the retarded (or advanced) Green function of a free electron in the potential (2). Note that the integral over $t^{\prime}$ in (13) is formally divergent for $\operatorname{Im} \epsilon<0$; it is to be understood as the analytic continuation from the upper half-plane of complex $\epsilon$, where $\operatorname{Im} \epsilon>0$. The Green functions $G^{( \pm)}$may be presented in the well-known Feynman form (in scaled units):

$$
G^{( \pm)}\left(\boldsymbol{r}, t, \boldsymbol{r}^{\prime}, t^{\prime}\right)=\mp \frac{\mathrm{i} \Theta\left[ \pm\left(t-t^{\prime}\right)\right]}{\left[4 \pi \mathrm{i}\left(t-t^{\prime}\right)\right]^{3 / 2}} \mathrm{e}^{\mathrm{i} S_{c l}\left(\boldsymbol{r}, t, \boldsymbol{r}^{\prime}, t^{\prime}\right)},
$$

where $\Theta(x)$ is the Heaviside function, and $S_{c l}$ is the classical action:

$S_{c l}\left(\boldsymbol{r}, t, \boldsymbol{r}^{\prime}, t^{\prime}\right)=\frac{\left(\boldsymbol{r}-\boldsymbol{r}^{\prime}\right)^{2}}{4\left(t-t^{\prime}\right)}-\frac{\left(\boldsymbol{r}-\boldsymbol{r}^{\prime}\right)}{\omega^{2}\left(t-t^{\prime}\right)} \cdot\left(\boldsymbol{F}(\omega t)-\boldsymbol{F}\left(\omega t^{\prime}\right)\right)+S_{c l}\left(t, t^{\prime}\right)$,

where

$$
\begin{aligned}
S_{c l}\left(t, t^{\prime}\right) \equiv S_{c l}(\boldsymbol{r} & \left.=0, t, \boldsymbol{r}^{\prime}=0, t^{\prime}\right)=-\frac{u_{p}}{\omega}\left[\omega\left(t-t^{\prime}\right)\left(1-\frac{4 \sin ^{2}\left(\omega\left(t-t^{\prime}\right) / 2\right)}{\left(\omega\left(t-t^{\prime}\right)\right)^{2}}\right)\right. \\
- & \left.l \cos \omega\left(t+t^{\prime}\right)\left(\sin \omega\left(t-t^{\prime}\right)-\frac{4 \sin ^{2}\left(\omega\left(t-t^{\prime}\right) / 2\right)}{\omega\left(t-t^{\prime}\right)}\right)\right] .
\end{aligned}
$$

Here the parameter $u_{p}$ is the ratio of the quiver energy of an electron in a laser field (the 'ponderomotive shift', $U_{p}$ ) to the binding energy $\left|E_{0}\right|$, i.e. the scaled $U_{p}$ shift; it is related to the well-known Keldysh parameter, $\gamma=\omega / F$, of strong field theories as:

$$
u_{p}=\frac{F^{2}}{2 \omega^{2}}=\left(2 \gamma^{2}\right)^{-1} \quad\left(=\frac{e^{2} F_{a b s}^{2}}{4 m \omega_{a b s}{ }^{2}\left|E_{0}\right|}=\frac{U_{p}}{\left|E_{0}\right|} \text { in absolute units }\right) .
$$

As for the case of quasistationary (or resonance) states in radiationless atomic problems, the QQES wavefunctions are non-normalizable by standard procedures because of their 
asymptotically divergent terms in $r$ (in open ionization channels). The proper normalization is achieved by introducing the 'dual functions', $\tilde{\Phi}_{\epsilon}(r, t)$, which provide the proper normalization of $\Phi_{\epsilon}$ in accordance with the relation

$$
\left\langle\left\langle\tilde{\Phi}_{\epsilon}(\boldsymbol{r}, t) \mid \Phi_{\epsilon}(\boldsymbol{r}, t)\right\rangle\right\rangle=\frac{1}{T} \int_{0}^{T} \mathrm{~d} t \int \mathrm{d} \boldsymbol{r} \tilde{\Phi}_{\epsilon}(\boldsymbol{r}, t)^{*} \Phi_{\epsilon}(\boldsymbol{r}, t)=1 .
$$

As discussed in $[29,144]$, the dual function is defined as follows:

$$
\tilde{\Phi}_{\epsilon}(\boldsymbol{r}, t)=[\Phi(\boldsymbol{r},-t)]_{\xi \rightarrow-\xi}^{*}
$$

Thus, the function $\left\langle\tilde{\Phi}_{\epsilon}\right| \equiv \tilde{\Phi}_{\epsilon}^{*}=\Phi(\boldsymbol{r},-t ;-\xi)$ may be considered as a 'bra-analogue' of $\left|\Phi_{\epsilon}\right\rangle \equiv \Phi(r, t ; \xi)$ and it should be used (instead of $\left.\left\langle\Phi_{\epsilon}\right|\right)$ in calculations of the normalization factor and, therefore, of matrix elements of operators acting on $\Phi_{\epsilon}(r, t)$.

\subsection{Basic equations for the QQES solution for a $\delta$-model potential}

The direct numerical solution of the eigenvalue integral equation (13) for a real atomic potential $U(r)$ is not a simple problem (though it is possible—see, e.g., [91]). However, this equation simplifies drastically for the $\delta$-model potential, taking into account the known behaviour at the origin for a solution of the Schrödinger equation involving this short-range potential [139]:

$$
\left.\Phi_{\epsilon}(\boldsymbol{r}, t)\right|_{r \rightarrow 0}=\left(\frac{1}{r}-1\right) f_{\epsilon}(t) .
$$

The QQES result for this case may be presented in the following form [127]:

$$
\Phi_{\epsilon}(\boldsymbol{r}, t)=-4 \pi \int_{0}^{\infty} \mathrm{e}^{\mathrm{i} \epsilon \tau} G^{(+)}(\boldsymbol{r}, t, 0, t-\tau) f_{\epsilon}(t-\tau) \mathrm{d} \tau,
$$

where $\epsilon$ is the eigenvalue of the one-dimensional integral equation for the periodic in time function $f_{\epsilon}(t)$

$$
(\sqrt{E}-1) f_{\epsilon}(t)=(4 \pi \mathrm{i})^{-1 / 2} \int_{0}^{\infty} \frac{\mathrm{d} \tau}{\tau^{3 / 2}} \mathrm{e}^{-\mathrm{i} E \tau}\left\{f_{\epsilon}(t-\tau) \mathrm{e}^{\mathrm{i} u_{p} \tau+\mathrm{i} S_{c l}(\tau, t-\tau)}-f_{\epsilon}(t)\right\},
$$

where $E=u_{p}-\epsilon$ and $S_{c l}\left(t, t^{\prime}\right)$ is defined by (16). Obviously, $f_{\epsilon}(t)$ is determined only by the S-wave part of $\Phi_{\epsilon}(r, t)$ at the origin, and it tends to $N=1 / \sqrt{2 \pi}$ (see (9)) at $F \rightarrow 0$. In view of the selection rules for dipole transitions, the Fourier expansion of $f_{\epsilon}(t)$ involves only harmonics of the same parity, as is evident from the explicit form of $S_{c l}(t, t-\tau)$ in (16). Moreover, analysis of equation (16) shows that (for not too small $\omega$ ) it is convenient to introduce a new function, $\phi_{\epsilon}(t)$, instead of $f_{\epsilon}(t)$ :

$$
f_{\epsilon}(t)=\sum_{n} f_{n} \mathrm{e}^{-2 \mathrm{i} n \omega t}=\exp \left[\mathrm{i} \frac{l u_{p}}{2 \omega} \sin 2 \omega t\right] \phi_{\epsilon}(t)=\exp \left[\mathrm{i} \frac{l u_{p}}{2 \omega} \sin 2 \omega t\right] \sum_{k} \phi_{k} \mathrm{e}^{-2 \mathrm{i} k \omega t} \text {. }
$$

In fact, this substitution is equivalent to the unitary transformation of the Schrödinger equation that removes the periodic in time part of the term $\propto A^{2}(t)$ in the photon-atom interaction (2). Obviously, the Fourier coefficients of $f_{\epsilon}(t), f_{n}$, and the $\phi_{n}$ coefficients are related in general by simple transformations:

$$
f_{n}=\sum_{k=-\infty}^{\infty} J_{k-n}\left(\frac{l u_{p}}{2 \omega}\right) \phi_{k}, \quad \phi_{k}=\sum_{n=-\infty}^{\infty} J_{k-n}\left(\frac{l u_{p}}{2 \omega}\right) f_{n} .
$$

The final eigenvalue equation for $\epsilon$ and $\phi_{\epsilon}(t)$ is [127]

$(\sqrt{E}-1) \phi_{\epsilon}(t)=(4 \pi \mathrm{i})^{-1 / 2} \int_{0}^{\infty} \frac{\mathrm{d} \tau}{\tau^{3 / 2}} \mathrm{e}^{-\mathrm{i} E \tau}\left\{\phi_{\epsilon}(t-\tau) \mathrm{e}^{\mathrm{i} z(\tau)[1-l \cos (\omega(2 t-\tau))]}-\phi_{\epsilon}(t)\right\}$, 
where

$$
z(\tau)=\frac{4 u_{p}}{\omega} \frac{\sin ^{2} \omega \tau / 2}{\omega \tau} .
$$

Equation (25) is equivalent to an infinite system of linear homogeneous equations for the Fourier coefficients $\phi_{n}$ :

$$
(\sqrt{E-2 n \omega}-1) \phi_{n}=\sum_{n^{\prime}=-\infty}^{\infty} M_{n, n^{\prime}}(E) \phi_{n^{\prime}}
$$

and a transcendental equation (the Fredholm determinant) for the complex quasienergy:

$$
\operatorname{det}\left\|(\sqrt{E-2 n \omega}-1) \delta_{n, n^{\prime}}-M_{n, n^{\prime}}(E)\right\|=0,
$$

with the boundary condition $\epsilon=E_{0}=-1$ at $F=0$. The matrix elements $M_{n, n^{\prime}}$ are integrals of Bessel functions $J_{m}(x)$ :

$$
M_{n, n^{\prime}}(E)=\frac{\mathrm{i}^{n-n^{\prime}}}{\sqrt{4 \pi \mathrm{i}}} \int_{0}^{\infty} \frac{\mathrm{d} \tau}{\tau^{3 / 2}} \mathrm{e}^{-\mathrm{i}\left(E-\left(n+n^{\prime}\right) \omega\right) \tau}\left[\mathrm{e}^{\mathrm{i} z(\tau)} J_{n^{\prime}-n}(l z(\tau))-\delta_{n, n^{\prime}}\right] .
$$

In actual calculations only the matrix elements $M_{0, n}(E-2 k \omega)$ with $n \geqslant 0$ are necessary in view of the symmetry relations obvious from (29):

$M_{n, n^{\prime}}(E, \omega)=M_{n^{\prime}, n}(E, \omega)=M_{-n,-n^{\prime}}(E,-\omega)=M_{n+k, n^{\prime}+k}(E+2 k \omega, \omega)$.

For both perturbative and nonperturbative calculations of the complex quasienergy for not too strong fields, the 'BW series' for $\epsilon$ [127] may be useful:

$$
\begin{aligned}
\sqrt{E}-1=M_{0,0}(E)+\sum_{n \neq 0} \frac{M_{0, n}(E) M_{n, 0}(E)}{\sqrt{E-2 n \omega}-1-M_{n, n}(E)}+\sum_{n \neq 0} \sum_{m \neq 0, m \neq n} \\
\times \frac{M_{0, n}(E) M_{n, m}(E) M_{m, 0}(E)}{\left(\sqrt{E-2 n \omega}-1-M_{n, n}(E)\right)\left(\sqrt{E-2 m \omega}-1-M_{m, m}(E)\right)}+\cdots,
\end{aligned}
$$

which is obtained by an iterative solution of the linear equation (27). Equation (31) is equivalent to the exact equation (28) for $\epsilon$.

Obviously, the total decay rate, $\Gamma=-2 \operatorname{Im} \epsilon$, is independent of an explicit form of the QQES $\Phi_{\epsilon}(\boldsymbol{r}, t)$. On the contrary, for calculations of partial $n$-photon rates and for the ADs of detached electrons (as well as for other applications of the QQES approach) the properly normalized function $\Phi_{\epsilon}(r, t)$ is necessary (see, e.g., [144]). For the $\delta$-model potential, the normalization of the QQES (21) according to the relation (18) is described in appendix A. For practical implementations of the (normalized) QQES solution (21) for the $\delta$-model potential it is first necessary to calculate the coefficients $\phi_{n}$ (or $f_{n}$ ) and the complex quasienergy $\epsilon$, e.g., according to (27) and (28). Detailed strong field and perturbative analyses of these key ingredients of the QQES approach for the $\delta$-model potential are presented in appendix B.

\subsection{Exact result for multiphoton detachment rates}

We will define the $n$-photon detachment amplitude using the asymptotic form of the normalized QQES wavefunction at a large distance from an ion. To avoid problems stemming from the long-range character of the standard representation (2) for the electron-laser dipole interaction, it is convenient for our present purposes to carry out a few unitary transformations for $\Phi_{\epsilon}(r, t)$. First, the transformation

$$
\Phi_{\epsilon}{ }^{(1)}(\boldsymbol{r}, t)=\exp \left[\frac{\mathrm{i}}{\omega^{4}} \int^{t}\left(\frac{\partial \boldsymbol{F}(\omega \tau)}{\partial \tau}\right)^{2} \mathrm{~d} \tau-\mathrm{i} u_{p} t\right] \Phi_{\epsilon}(\boldsymbol{r}, t)
$$


removes the periodic in time part of the term $\sim A^{2}(t)$ from the Hamiltonian $\mathcal{H}$. This is equivalent to using equation (21) for $\Phi_{\epsilon}^{(1)}(\boldsymbol{r}, t)$ with $f_{\epsilon}(t) \rightarrow \phi_{\epsilon}(t)$ (and equation (A.1) for $\tilde{\Phi}_{\epsilon}^{(1)}(\boldsymbol{r}, t)$ with an analogous substitution for $\left.\tilde{f}_{\epsilon}(t)\right)$. Then we transform to the frame oscillating with the laser field by introducing a new position vector, $\boldsymbol{R}$ :

$$
\boldsymbol{r}=\boldsymbol{R}+\frac{2}{\omega^{2}} \boldsymbol{F}(\omega t)
$$

which removes the term $\sim \boldsymbol{A}(t) \cdot \hat{\boldsymbol{p}}$ from $\mathcal{H}$, so that the entire laser-field dependence is concentrated in the potential $U\left(\boldsymbol{R}+\frac{2}{\omega^{2}} \boldsymbol{F}(\omega t)\right)$. This corresponds to the well-known Kramers-Henneberger transformation [145]. The result is that the QQES wavefunction (21) is transformed to

$$
\begin{aligned}
\psi_{E}(\boldsymbol{R}, t) \equiv \Phi_{\epsilon}^{(1)} & \left(\boldsymbol{R}+\frac{2}{\omega^{2}} \boldsymbol{F}(\omega t), t\right)=\frac{1}{\sqrt{4 \pi \mathrm{i}}} \sum_{k=-\infty}^{\infty} \phi_{k} \\
& \times \int_{0}^{\infty} \frac{\mathrm{d} \tau}{\tau^{3 / 2}} \exp \left\{\mathrm{i}\left[2 k \omega(\tau-t)-E \tau+\frac{1}{4 \tau}\left(\boldsymbol{R}+\frac{2}{\omega^{2}} \boldsymbol{F}(\omega t-\omega \tau)\right)^{2}\right]\right\} .
\end{aligned}
$$

The $n$th Fourier coefficient of $\psi_{E}$ has the following asymptotic behaviour at $R=|\boldsymbol{R}| \rightarrow \infty$ (see appendix C):

$$
\lim _{R \rightarrow \infty} \frac{1}{T} \int_{0}^{T} \mathrm{e}^{\mathrm{i} n \omega t} \psi_{E}(\boldsymbol{R}, t) \mathrm{d} t \longrightarrow \mathcal{A}_{n} \frac{\mathrm{e}^{\mathrm{i} k_{n} R}}{R}, \quad T=2 \pi / \omega,
$$

where $k_{n}^{2}=n \omega-E=\epsilon+n \omega-u_{p}$ is complex because $\epsilon$ is complex. The open $n$-photon detachment channels correspond to $\operatorname{Re} k_{n}^{2}>0$. Obviously, $\mathcal{A}_{n}$ depends on the direction $\boldsymbol{n}=\boldsymbol{R} / R$, but not on $R$. The explicit form of $\mathcal{A}_{n}$ is (see appendix C)

$$
\mathcal{A}_{n}=\mathrm{i}^{n} \sum_{p=-\infty}^{\infty}(-1)^{p} \phi_{p} J_{n-2 p}\left(\frac{2 F k_{n}}{\omega^{2}}|\boldsymbol{e} \cdot \boldsymbol{n}|\right)\left(\frac{\boldsymbol{e} \cdot \boldsymbol{n}}{|\boldsymbol{e} \cdot \boldsymbol{n}|}\right)^{n-2 p} .
$$

The lowest, $n_{0}$ th, open channel is determined by the dynamical threshold condition, $n_{0}=$ $1+\left[|\operatorname{Re} \epsilon|+u_{p}\right] / \omega$, where $[x]$ is the largest integer less than $x$. Thus it depends on both the frequency and the intensity of the laser field. For open channels, Re $k_{n}>0$, whereas for a closed channel $m$ (i.e. for $m<\left[|\operatorname{Re} \epsilon|+u_{p}\right] / \omega$ ) the branch of the square root is defined by the condition $\operatorname{Im} k_{m}>0$. Finally, taking into account (24), the exact amplitude (36) may be presented in terms of the coefficients $f_{n}$ :

$\mathcal{A}_{n}=\mathrm{i}^{n} \sum_{k=-\infty}^{\infty} f_{k} \sum_{p=-\infty}^{\infty}(-1)^{p} J_{p-k}\left(\frac{l u_{p}}{2 \omega}\right) J_{n-2 p}\left(\frac{2 F k_{n}}{\omega^{2}}|\boldsymbol{e} \cdot \boldsymbol{n}|\right)\left(\frac{\boldsymbol{e} \cdot \boldsymbol{n}}{|\boldsymbol{e} \cdot \boldsymbol{n}|}\right)^{n-2 p}$.

The differential rate for $n$-photon detachment with detection of the detached electrons in the direction $\boldsymbol{n}$ may be defined as

$$
\frac{\mathrm{d} \Gamma^{(n)}}{\mathrm{d} \Omega} \equiv \Gamma^{(n)}(\boldsymbol{n})=2\left|\sqrt{k_{n}} \mathcal{A}_{n}\right|^{2},
$$

where $\mathrm{d} \Omega \equiv \mathrm{d} \boldsymbol{n}$. For real $k_{n}$ (neglecting the imaginary part of $\epsilon$ ) this definition coincides with the standard definition for the angular distribution of detached electrons having the asymptotic momentum, $\boldsymbol{p}_{\boldsymbol{n}}=k_{n} \boldsymbol{n}$, in terms of the electron flux. This formulation, however, cannot be used directly for the quasistationary states. Thus, the definition (38) may be considered as an analytical continuation of the standard definition for the case of the QQES approach. (Below in section 2.6.4 we present some arguments to justify our definition (38).) Using in (36) the relations (6) and (7), we can express the dependence of the differential rate (38) in terms of the spherical angles of $n, \theta$ and $\varphi$. Then the total detachment rate for absorption of $n$ photons, 
$\Gamma^{(n)}$, is obtained by simply integrating equation (38) over all solid angles. Summing over all possible numbers of absorbed photons, one obtains then the total detachment rate:

$$
\sum_{n=n_{0}}^{\infty} \Gamma^{(n)}=\Gamma .
$$

\subsection{The QQES wavefunction and detachment rates in the Keldysh approximation}

The QQES solution discussed in previous sections provides a rare opportunity to compare rigorously derived results of an exactly solvable problem with results obtained using the Keldysh approximation (KA), which is a limiting case. It is well known that, in general, the KA is applicable for low frequencies, $\omega \ll 1$, and for sufficiently strong $F(\omega \ll F \ll 1)$ such that the Keldysh parameter, $\gamma=\omega / F$, is small compared to unity. Thus, following the analysis performed in [127], we consider the low frequency limit of the exact equations for $\epsilon$ and $\Phi_{\epsilon}(\boldsymbol{r}, t)$. First of all, for small $F$, it is reasonable to expect that the function $f_{\epsilon}(t)$ in the boundary condition (20) (and therefore in the integral equation (22)) depends only weakly on the small parameter $\omega$, i.e. it is reasonable to start from the results for $\epsilon$ and $\Phi_{\epsilon}(\boldsymbol{r}, t)$ at $f_{\epsilon}(t) \approx$ constant. On the other hand, the time dependence of $\phi_{\epsilon}(t)$ may be significant even for small $\omega$ in view of the exponential factor in the substitution (23). Thus, for small $\omega$, it is convenient to use the QQES equations in terms of coefficients $f_{n}$ instead of $\phi_{n}$ (cf (23)). It follows from equations (22), (23) and (25) that the coefficients $f_{n}$ satisfy equations similar to those satisfied by $\phi_{n}$ (i.e. equations (27)-(31) as well as the normalization condition (A.3)), provided one makes the substitution $M_{n n^{\prime}}(E) \rightarrow \tilde{M}_{n n^{\prime}}(E)$, where the integral for $\tilde{M}_{n n^{\prime}}(E)$ differs from that in (29) by the following substitution in the argument of the Bessel function in (29):

$$
l z(\tau) \rightarrow l\left[z(\tau)-\left(u_{p} / \omega\right) \sin \omega \tau\right] .
$$

As shown in [127], to calculate $\epsilon$ for small $\omega$ it is sufficient to approximate the full BW expansion (obtained by substituting $M_{n n^{\prime}}(E) \rightarrow \tilde{M}_{n n^{\prime}}(E)$ in equation (31)) by its leading term:

$$
\sqrt{E}=1+\tilde{M}_{00}(E) \text {. }
$$

In terms of $f_{\epsilon}(t)$, the approximation (41) corresponds to the substitution $f_{\epsilon}(t) \approx$ constant in equation (22) with subsequent averaging of the rhs of this equation over the laser period $T=2 \pi / \omega$. Equivalently, it corresponds to neglecting coefficients $f_{n}$ with $n \neq 0$ in (22). Putting $E=1+u_{p}$ on the rhs of (41) and using the explicit form of $\tilde{M}_{00}\left(1+u_{p}\right)$ in terms of a cycle-averaged integral over time of the Volkov Green function, the imaginary part of $\epsilon$ may be obtained as [127]

$$
\begin{aligned}
\Gamma & =-2 \operatorname{Im} \epsilon=2 \operatorname{Im}\left(2 \tilde{M}_{00}+\tilde{M}_{00}^{2}\right) \approx 4 \operatorname{Im} \tilde{M}_{00}\left(1+u_{p}\right) \\
& =8 \pi \sum_{n} \int \mathrm{d} \boldsymbol{p}\left|F_{n}(\boldsymbol{p})\right|^{2} \delta\left(\boldsymbol{p}^{2}+1+u_{p}-n \omega\right),
\end{aligned}
$$

where $F_{n}(\boldsymbol{p})$ is the Fourier coefficient of the Volkov wavefunction at the origin, $\varphi_{p}(\boldsymbol{r}=0, t)$. The result (42) coincides exactly with the initial formula for the decay rates in the KA (see, e.g., equations (10)-(15) in [1](c)). KA calculations of $\Gamma$ for $\mathrm{H}^{-}$based on the direct evaluation of $\operatorname{Im} \tilde{M}_{00}$ in (42) were carried out in [146]. Many authors have estimated KA decay rates, based upon the formulation in [1], using a saddle-point analysis for the coefficients $F_{n}(p)$ (which in general may be expressed in terms of the so-called 'generalized' Bessel functions [1](b), [147]; see also (45) below). More general results, also using a saddle-point analysis, were obtained in [128] for the case of linear laser polarization. The results obtained from this latter analysis are in good agreement with experimental measurements and with results of other calculations 
up to unexpectedly high frequencies of about $\omega \sim(0.3-0.5)$. This is not surprising, since the analysis in [127] shows that (for not too strong $F$ ) the approximation (41) results in a fractional error $(\Delta \Gamma / \Gamma)$ in the calculated value for $\Gamma$ that is of the order of $\omega / 16$.

In summary, the analytic expressions of the Keldysh approach, both for the wavefunctions and the decay rates, follow from the exact QQES results by approximating $\epsilon \approx E_{0}=-1$, neglecting the Fourier coefficients $f_{k}$ with $k \neq 0$ and by using either of the equivalent approximations (41) or (42) to calculate the total decay rates. The QQES wavefunction (21) reduces in the KA to

$$
\Phi_{K A}(\boldsymbol{r}, t)=-\sqrt{8 \pi} \int_{0}^{\infty} \mathrm{e}^{-\mathrm{i} \tau} G^{(+)}(\boldsymbol{r}, t, 0, t-\tau) \mathrm{d} \tau,
$$

where we have used $f_{0}=1 / \sqrt{2 \pi}$ so that, for $F \rightarrow 0$, (43) yields the initial state (9). Finally, as follows from (24), the coefficients $\phi_{k}$ in the KA reduce to Bessel functions

$$
\phi_{k}=\frac{1}{\sqrt{2 \pi}} J_{k}\left(\frac{l u_{p}}{2 \omega}\right) .
$$

Note that in the KA (i.e. using (44) and the approximation $\epsilon=-1$ in $k_{n}$ ) the exact result (36) for $\mathcal{A}_{n}$ reduces to

$$
\mathcal{A}_{n}^{K A}=\frac{\mathrm{i}^{n}}{\sqrt{2 \pi}} \sum_{p=-\infty}^{\infty} J_{p}\left(l \frac{u_{p}}{2 \omega}\right) J_{2 p+n}\left(\frac{2 F k_{n}}{\omega^{2}}|e \cdot n|\right)\left(\frac{e \cdot n}{|e \cdot n|}\right)^{2 p+n} .
$$

For the cases of linear and circular laser polarizations this result coincides with that obtained by Reiss [147]. Note that the sum over products of Bessel functions in (45) defines the socalled 'generalized' Bessel function, which also has a one-dimensional integral representation (see [1](b), [147]). For an initial $S$-state and a linearly polarized field, Gribakin and Kuchiev [128] obtained an approximation to the integral form of the 'generalized' Bessel function using a saddle-point (adiabatic) analysis. Obviously, the result (45) is equivalent to that in (37) for $f_{k}=\delta_{k 0} / \sqrt{2 \pi}$. Finally, with the use of the approximation I (B.31) for the coefficients $f_{k}$ (see appendix B.4), equation (37) with $f_{k} \rightarrow f_{k}^{(1)}$ gives the amplitude $\mathcal{A}_{n}$ taking account (to first order) of the binding potential ('rescattering') correction to the KA result (45). Note that the use of the approximation II for $f_{k}$ (see (B.32)), i.e. $f_{k} \rightarrow f_{k}^{(2)}$, yields the result for $\mathcal{A}_{n}$ equivalent to that in the so-called 'improved' KA [133].

\subsection{Analytical PT results for angular distributions and n-photon rates}

The exact results (36) and (38) permit a simple analytical analysis for weak fields. In contrast to the case of laser ionization of atoms, for the case of multiphoton detachment of negative ions (by Nd:YAG or higher-frequency lasers) the PT regime is relevant to most existing experiments. Indeed, the results of recent many-electron PT calculations [114] are in good agreement with experimental results for detachment of negative halide ions. Also, existing studies of ellipticity effects in multiphoton detachment (see [74-77]) employ LOPT. Analytical PT results have the advantage that they allow one to carry out an exhaustive analysis of ADs over a wide interval of frequencies.

The analytical PT expressions for $\Gamma^{(n)}(\boldsymbol{n})$ (including higher-order corrections to the LOPT result) may be derived by an expansion of the Bessel functions and coefficients $\phi_{k}$ in (36) in a power series in $F$. Obviously, in order to calculate higher-order (in $F$ ) corrections to ADs, a proper account of intensity-dependent corrections to the normalization factor $\phi_{0}^{2}$ and the coefficients $\phi_{k}$ with $k \neq 0$ in (36) is required. These corrections are presented in appendices B.1 and B.2 for both BW and RS versions of PT. In principle, these results allow us to investigate analytically both LOPT and higher-order PT effects for the general 
case of $n$-photon detachment, although final results for high-order effects do involve rather cumbersome combinations of the $\mathcal{D}_{m}(E+p \omega)$ functions (see appendix B.2). As an example of the analytical structure of high-order PT corrections for $n>1$, we present below only the linear in $I$ correction for two-photon detachment. (For results for $n=1$, see section 5.1.)

2.6.1. Two-photon detachment. The exact strong field result for the differential rate of twophoton detachment is

$$
\Gamma^{(2)}(\boldsymbol{n})=2\left|(2 \omega-E)^{1 / 4} \mathcal{A}_{2}\right|^{2},
$$

where $\mathcal{A}_{2}$ is given by (36). Expanding the Bessel function in (36) in a power series in $F$, the BW result for $\mathcal{A}_{2}$, taking into account terms $\sim F^{2}$ and $F^{4}$, is

$$
\begin{gathered}
\mathcal{A}_{2}=-\frac{\phi_{0}}{2}\left(\frac{F}{\omega^{2}}\right)^{2}(2 \omega-E)(e \cdot n)^{2}\left[1-(2 \omega-E)\left(\frac{F}{\omega^{2}}\right)^{2} \frac{|e \cdot n|^{2}}{3}\right] \\
+\phi_{1}\left[1-(2 \omega-E)\left(\frac{F}{\omega^{2}}\right)^{2}|e \cdot n|^{2}\right],
\end{gathered}
$$

where $\phi_{1}$ and $\phi_{0}^{2}$ are given by (B.5) and (B.8). The two-photon differential rate, including the linear-in-intensity correction to the lowest-order BW result, follows from (46):

$\Gamma_{B W}^{(2)}(\boldsymbol{n})=\frac{1}{2}\left|(2 \omega-E)^{1 / 4} \phi_{0}\right|^{2}\left(\frac{F}{\omega^{2}}\right)^{4}\left\{|A|^{2}-2\left(\frac{F}{\omega^{2}}\right)^{2} \operatorname{Re}\left(A^{*} B\right)\right\}$,

where

$$
\begin{aligned}
A=(2 \omega-E)(e \cdot \boldsymbol{n})^{2}+\frac{l \mathcal{D}_{1}(E-\omega)}{\sqrt{E-2 \omega}-1-M_{1,1}(E)} \\
B=\frac{1}{3}(2 \omega-E)^{2}|\boldsymbol{e} \cdot \boldsymbol{n}|^{2}(\boldsymbol{e} \cdot \boldsymbol{n})^{2}+\frac{l}{\sqrt{E-2 \omega}-1-M_{1,1}(E)} \\
\times\left[(2 \omega-E) \mathcal{D}_{1}(E-\omega)|\boldsymbol{e} \cdot \boldsymbol{n}|^{2}-\mathcal{D}_{2}(E-\omega)-\frac{\mathcal{D}_{1}(E-\omega) \mathcal{D}_{1}(E-2 \omega)}{\sqrt{E-2 \omega}-1-M_{1,1}(E)}\right] .
\end{aligned}
$$

Note that the correction term $B$ involves a more complicated polarization angular factor, $|e \cdot n|^{2}(e \cdot n)^{2}$, than that in the zero-order term $A$. Keeping only terms $\sim F^{2}$ in (47), we have that $E \simeq 1, \phi_{0} \simeq 1 / \sqrt{2 \pi}$ and $\phi_{1}$ is given by (B.20). Thus the RS LOPT result for $\Gamma^{(2)}(\boldsymbol{n})$ has a simple form:

$$
\Gamma^{(2)}(\boldsymbol{n})=\frac{F^{4}}{4 \pi \omega^{8}} \sqrt{2 \omega-1}\left|(2 \omega-1)(\boldsymbol{e} \cdot \boldsymbol{n})^{2}-l \frac{\mathrm{i}(2 \omega-1)^{3 / 2}-2 \mathrm{i}(\omega-1)^{3 / 2}+1}{3(1+\mathrm{i} \sqrt{2 \omega-1})}\right|^{2} .
$$

This expression coincides with that obtained in [49] by a direct second-order PT calculation for a $\delta$-model potential. Integrating (49) over the directions of $\boldsymbol{n}$, one obtains the total two-photon decay rate in LOPT:

$$
\Gamma^{(2)}=\frac{2}{45} \frac{F^{4}}{\omega^{8}} \sqrt{2 \omega-1}\left\{3(2 \omega-1)^{2} \xi^{2}+l^{2}\left[2(2 \omega-1)^{2}+\frac{5}{\omega}(\omega-1)^{2}|1+\mathrm{i} \sqrt{\omega-1}|^{2}\right]\right\},
$$

which coincides with that obtained previously in [81] (see also [80, 88] for the particular cases of linear and circular polarizations $(l=1$ and 0$))$.

In figure 2 we compare the LOPT result for $\Gamma^{(2)}$ with both the (more accurate) BW result and the exact result. Figure 2(a) illustrates the frequency dependence of $\Gamma^{(2)}$ for an intensity $I=0.04$ in two levels of approximation: (i) the RS LOPT result, (50), and (ii) the BW result including corrections $\sim I$ and $I^{2}$ to the LOPT BW result. (Note that we have not presented in this paper the analytic expression for the BW rate including correction terms $\sim I^{2}$; the result 

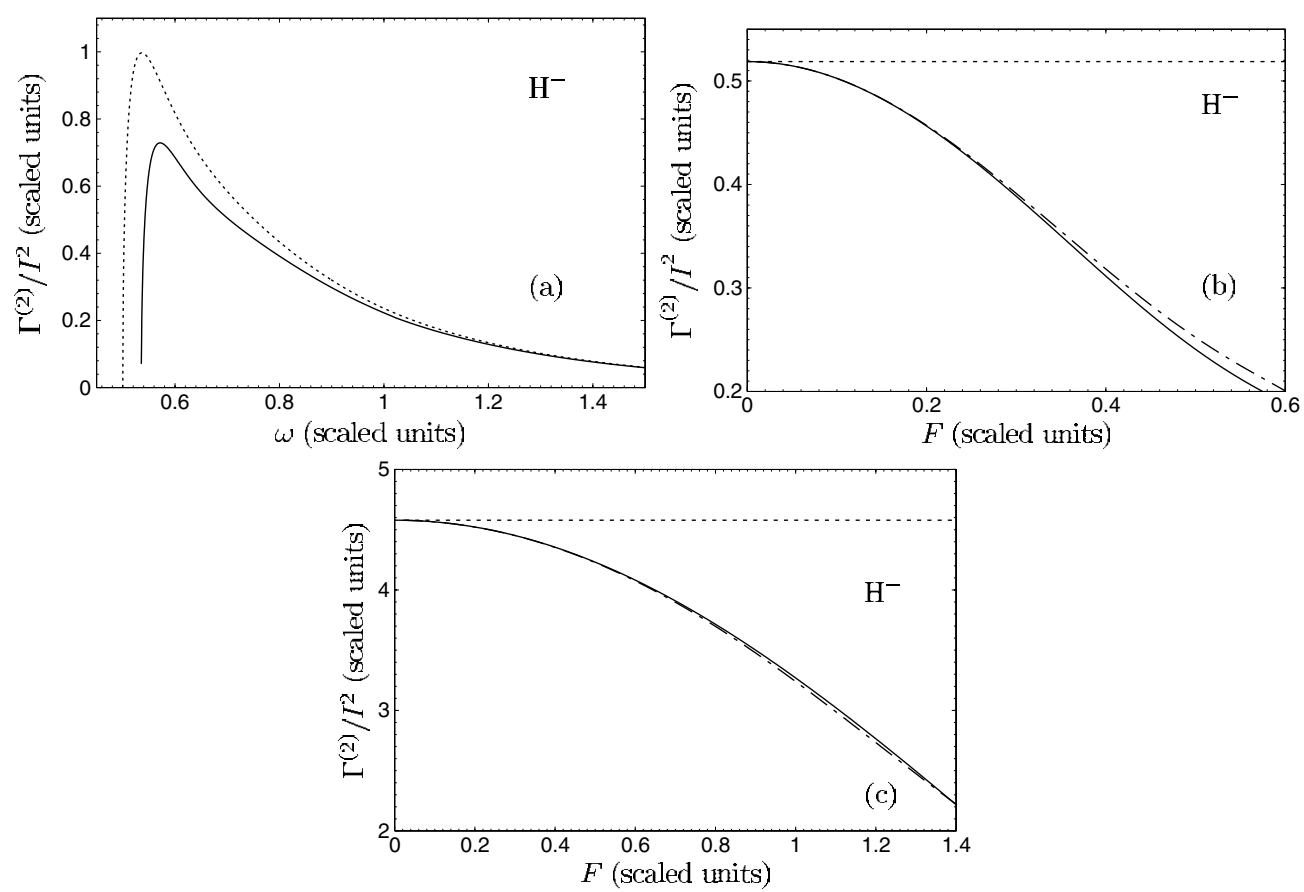

Figure 2. Total rate of two-photon detachment for $\mathrm{H}^{-}$(divided by $I^{2}$ in scaled units) in a linearly polarized laser field. Short broken curve: the LOPT result, (50); full curve: the BW result, $\Gamma_{B W}^{(2)}$, including the corrections $\sim I$ and $I^{2}$; chain curve: exact result. (a) Frequency dependence for $F=0.2$ (the exact and BW results are indistinguishable in this figure). (b) Field dependence for the below-threshold frequency, $\omega=0.74$. (c) Field dependence for the above-threshold frequency, $\omega=1.61$.

including the correction $\sim I$ is obtained by integration of (48) over the angles of $n$.) The results for $n=2$ shown in figure 2 are qualitatively similar to those for the case of $n=1$ (see section 5 below), although the deviations from LOPT results take place at lower intensities than for $n=1$. As shown in figure 2, with increasing $F$, the percentage reduction of the BW rate, $\Gamma_{B W}^{(2)}$, below the LOPT result is much greater for $\omega<1$ than for above-threshold frequencies for a given change in $F$. Note also the close agreement of the BW results with the exact ones.

2.6.2. Results for $n=3-5$. Three-photon detachment can be analysed similarly to the case of $n=2$. Here

$$
\mathcal{A}_{3}=\frac{F}{\omega^{2}} \frac{\sqrt{3 \omega-E}(\boldsymbol{e} \cdot \boldsymbol{n})}{6}\left[6 \phi_{1}-\frac{\phi_{0} F^{2}}{\omega^{4}}(3 \omega-E)(e \cdot \boldsymbol{n})^{2}\right],
$$

and thus we have the following LOPT results for both differential and total rates:

$$
\begin{aligned}
& \Gamma^{(3)}(\boldsymbol{n})=\frac{1}{36 \pi}\left(\frac{F}{\omega^{2}}\right)^{6}|\boldsymbol{e} \cdot \boldsymbol{n}|^{2}(3 \omega-1)^{3 / 2} \\
& \times\left|(3 \omega-1)(e \cdot \boldsymbol{n})^{2}-l \frac{\mathrm{i}(2 \omega-1)^{3 / 2}-2 \mathrm{i}(\omega-1)^{3 / 2}+1}{1+\mathrm{i} \sqrt{2 \omega-1}}\right|^{2},
\end{aligned}
$$




$$
\begin{aligned}
\Gamma^{(3)}=\frac{2}{315}\left(\frac{F}{\omega^{2}}\right)^{6}(3 \omega-1)^{3 / 2}\left[\xi^{2}(3 \omega-1)^{2}\right. & \\
+ & \left.l^{2}\left(\frac{2}{5}(3 \omega-1)^{2}+\frac{35}{6}\left|\frac{\omega-2}{5}-2(\omega-1) \frac{1+\mathrm{i} \sqrt{\omega-1}}{1+\mathrm{i} \sqrt{2 \omega-1}}\right|^{2}\right)\right] .
\end{aligned}
$$

We present also the explicit forms of the differential rates for $n=4$ and 5:

$$
\begin{aligned}
\Gamma^{(4)}(\boldsymbol{n})= & \left(\frac{F}{\omega^{2}}\right)^{8} \frac{\sqrt{4 \omega-1}}{(4 !)^{2} \pi}\left|(4 \omega-1)^{2}(\boldsymbol{e} \cdot \boldsymbol{n})^{4}-2 l(\boldsymbol{e} \cdot \boldsymbol{n})^{2}(4 \omega-1) g_{1}(\omega)+\frac{2}{3} l^{2} g_{2}(\omega)\right|^{2}, \\
\Gamma^{(5)}(\boldsymbol{n})= & \left(\frac{F}{\omega^{2}}\right)^{10}|\boldsymbol{e} \cdot \boldsymbol{n}|^{2} \frac{(5 \omega-1)^{3 / 2}}{(5 !)^{2} \pi} \mid(5 \omega-1)^{2}(\boldsymbol{e} \cdot \boldsymbol{n})^{4} \\
& \quad-\frac{10}{3} l(\boldsymbol{e} \cdot \boldsymbol{n})^{2}(5 \omega-1) g_{1}(\omega)+\left.\frac{10}{3} l^{2} g_{2}(\omega)\right|^{2},
\end{aligned}
$$

where $g_{1}(\omega)$ and $g_{2}(\omega)$ enter the LOPT results via the coefficients $\phi_{1}$ and $\phi_{2}$ (cf (B.20)).

2.6.3. The multiphoton case. The general structure of the ADs for higher $n$ can be discerned from the results for $2 \leqslant n \leqslant 5$ presented above. The LOPT result for the amplitude $\mathcal{A}_{n}$ is obtained by expansion of the Bessel functions in (36) in power series in $F$, and by taking into account the explicit form (B.10) for the coefficients $\phi_{p}$ in LOPT. The final result for $\mathcal{A}_{n}^{\text {lopt }}$ has a simple form in terms of the LOPT coefficients $\chi_{k}(\omega)$ (see (B.10)):

$$
\mathcal{A}_{n}^{\text {lopt }}=\frac{1}{\sqrt{2 \pi}}\left(\frac{F}{\omega^{2}}\right)^{n} \sum_{k=0}^{[n / 2]}(-1)^{\frac{n-2 k}{2}} l^{k} \frac{(\boldsymbol{e} \cdot \boldsymbol{n})^{n-2 k}}{(n-2 k) !}(n \omega-1)^{\frac{n-2 k}{2}} \chi_{k}(\omega) .
$$

Note that, for both even and odd $n, n=2 m$ and $2 m+1$, the polarization-angular structure of the amplitude involves the same number, $m+1$, of terms with different polarization-angular factors $l^{k}(\boldsymbol{e} \cdot \boldsymbol{n})^{n-2 k}$. Note that the term with $k=0$ corresponds to the maximal angular momentum $L$ of the detached electron, $L=n$, and only this term (with $\chi_{0}(\omega)=1$ ) contributes to the amplitude for the case of circular polarization, $l=e \cdot e=0$. The differential rate in LOPT is given by (38) with $k_{n}=\sqrt{n \omega-1}$ :

$\Gamma_{\text {lopt }}^{(n)}(\boldsymbol{n})=\frac{1}{\pi}\left(\frac{F}{\omega^{2}}\right)^{2 n} \sqrt{n \omega-1}\left|\sum_{k=0}^{[n / 2]} \frac{(-l)^{k}}{(n-2 k) !}[(\boldsymbol{e} \cdot \boldsymbol{n}) \sqrt{n \omega-1}]^{n-2 k} \chi_{k}(\omega)\right|^{2}$.

Using the parametrization (7) for $\boldsymbol{e} \cdot \boldsymbol{n}$ in terms of $1 \pm \xi$, the integration of equation (57) over $\Omega$ is straightforward and yields the total rate:

$$
\begin{aligned}
\Gamma_{\text {lopt }}^{(n)}=4\left(\frac{F}{\omega^{2}}\right)^{2 n}(n \omega-1)^{n+1 / 2}\left[\sum_{k=0}^{[n / 2]} \frac{l^{2 k}}{(n-2 k) !} \sum_{k^{\prime}=0}^{k} \frac{4^{k^{\prime}-k}(1-n \omega)^{-2 k^{\prime}}\left|\chi_{k^{\prime}}(\omega)\right|^{2}}{\left(2 n-4 k^{\prime}+1\right) ! !\left[\left(k-k^{\prime}\right) !\right]^{2}}\right. \\
\left.+4 \sum_{k=1}^{[n / 2]} \sum_{k^{\prime}=0}^{k-1} \Phi_{n, k, k^{\prime}}\left(l^{2}\right) \frac{\left[\left(n-k-k^{\prime}\right) !\right]^{2}(1-n \omega)^{-k-k^{\prime}}}{\left(2\left(n-k-k^{\prime}\right)+1\right) !} \operatorname{Re}\left\{\chi_{k}(\omega) \chi_{k^{\prime}}^{*}(\omega)\right\}\right]
\end{aligned}
$$

The polarization-dependent factor $\Phi_{n, k, m}\left(l^{2}\right)$ is defined by

$$
\begin{aligned}
\Phi_{n, k, k^{\prime}}\left(l^{2}\right)= & \sum_{s=0}^{[n / 2]-k} l^{2(k+s)} \frac{{ }_{2} F_{1}\left(s+k-\frac{n}{2}, s+k-\frac{n-1}{2} ; \frac{1}{2} ; 1-l^{2}\right)}{s !\left(s+k-k^{\prime}\right) !(n-2 k-s) !\left(n-k-k^{\prime}-s\right) !} \\
& +\left(\frac{n-1}{2}-\left[\frac{n}{2}\right]\right) \frac{l^{n}}{\left[(n / 2-k) !\left(n / 2-k^{\prime}\right) !\right]^{2}},
\end{aligned}
$$


where the last term contributes only for even $n$, and where ${ }_{2} F_{1}$ is the hypergeometric polynomial. The cumbersome factor (59) vanishes for circular polarization $(l=0)$ and may be transformed to a simpler form for the case of linear polarization:

$$
\Phi_{n, k, k^{\prime}}(l=1)=\frac{\left(2 n-2 k-2 k^{\prime}\right) !}{2\left[\left(n-k-k^{\prime}\right) !\right]^{2}} .
$$

The amplitude (56) depends on $\boldsymbol{n}$ through even or odd powers of $\boldsymbol{e} \cdot \boldsymbol{n}$. In agreement with dipole selection rules, this means that for the detached electron only odd values of the angular momentum $L$ are allowed for odd $n$ and only even values of $L$ are allowed for even $n$. $L$ takes the values $L=L_{\text {min }}, L_{\min }+2, \ldots, n$, where $L_{\text {min }}=n-2[n / 2]$ is the minimal angular momentum of the detached electron for even $\left(L_{\min }=0\right)$ and odd $\left(L_{\min }=1\right)$ values of $n$. Partial decay rates into a fixed $L$ channel are useful, in particular, to estimate the quality of multielectron wavefunctions in accurate calculations of the multiphoton detachment rate (see, e.g., [105]). Partial amplitudes $\mathcal{A}_{n, L}^{\text {lopt }}$ may be obtained by projecting (56) onto the spherical harmonics, $Y_{L M}(\theta, \varphi)$, with the use of (6) and (7). For an elliptic polarization the general expression for $\mathcal{A}_{n, L}^{\text {lopt }}$ is rather cumbersome; thus, we present here only the results for linear polarization, in which case $\boldsymbol{e} \cdot \boldsymbol{n}=\hat{\epsilon} \cdot \boldsymbol{n}=\cos \alpha$ and the amplitude (56) involves only Legendre polynomials $P_{L}(\cos \alpha)$ :

$$
\mathcal{A}_{n, \text { lin }}^{\text {lopt }}=\frac{1}{\sqrt{4 \pi}}\left(\frac{F}{\omega^{2}}\right)^{n} \sum_{L=L_{\min }, L_{\min }+2, \ldots,}^{n} \sqrt{2 L+1} a_{L}(\omega) P_{L}(\cos \alpha) .
$$

The function $a_{L}(\omega)$ defines the partial amplitude for a given $L$ channel:

$a_{L}(\omega)=(-1)^{n / 2} \sqrt{2(2 L+1)} \sum_{k=0}^{(n-L) / 2} \frac{(-1)^{k}(n \omega-1)^{(n-2 k) / 2}}{(n-L-2 k) ! !(n+L+1-2 k) ! !} \chi_{k}(\omega)$.

The sum over $k$ in (62) corresponds to the coherent superposition of amplitudes for the detachment pathways with various combinations of angular momenta in intermediate states that contribute to the fixed- $L$ final state continuum channel. Thus, we obtain the following partial expansion for $\Gamma_{\text {lopt, lin }}^{(n)}$ :

$$
\Gamma_{\text {lopt }, \text { lin }}^{(n)}=\sum_{L=L_{\min }}^{n} \Gamma_{\text {lopt }, \text { lin }}^{(n), L}
$$

where the partial decay rate into the $L$ th channel is

$$
\Gamma_{\text {lopt }, \text { lin }}^{(n),}=2 \sqrt{n \omega-1}\left(\frac{F}{\omega^{2}}\right)^{2 n}\left|a_{L}(\omega)\right|^{2} .
$$

2.6.4. Threshold phenomena and connection of partial rates to the imaginary part of the quasienergy. The analytical PT results presented above permit a detailed analysis of all the frequency, polarization and angular dependences of multiphoton differential cross sections in a weak laser field. The frequency dependence of results for $n \geqslant 2$ with increasing $\omega$ above the $(n-1)$-photon threshold $(\omega \geqslant 1 /(n-1))$ exhibits threshold anomalies (TAs) (caused by branch-point non-analyticities of the $n$-photon amplitudes) at the thresholds for $(n-1)$, $(n-2), \ldots, 2$ and 1-photon detachment. Note that these above-threshold peculiarities do not occur in the case of purely circular polarization, $\xi= \pm 1$ (owing to the dipole selection rules for this case). On the contrary, for the case of linear polarization the non-analyticities that arise at the opening of intermediate state thresholds result in frequency dependences of total $n$-photon rates and AD parameters that vary considerably and differently on either side of each threshold. In particular, for $l=1$ the two-photon cross section (50) has quite different 
forms for 'above-threshold frequencies', $\omega>1,\left(\Gamma_{\text {ath }}^{(2)}\right)$, and for below-threshold frequencies, $\frac{1}{2}<\omega<1,\left(\Gamma_{b t h}^{(2)}\right)$ :

$\Gamma_{\text {ath }}^{(2)}=\frac{2}{45} \frac{F^{4}}{\omega^{8}} \sqrt{2 \omega-1}\left(13 \omega^{2}-18 \omega+7\right), \quad \omega>1$,

$\Gamma_{b t h}^{(2)}=\frac{2}{45} \frac{F^{4}}{\omega^{8}} \sqrt{2 \omega-1}\left\{3 \omega^{2}+12 \omega-23+\frac{10}{\omega}\left[1-(1-\omega)^{5 / 2}\right]\right\}, \quad \frac{1}{2}<\omega<1$.

Despite the non-analyticity, the frequency dependence of the $n$-photon cross sections is continuous at the thresholds; in particular, $\Gamma^{(2)}(\omega \rightarrow 1)=(2 / 45) F^{4}\left(3 \xi^{2}+2 l^{2}\right)$, regardless of whether $\omega \rightarrow 1$ from above or below $\omega=1$. This fact (as well as the very origin of the threshold peculiarities) may be seen most clearly from the 'scattering phase representation' for the $\phi_{k}(\omega)$ coefficients that is introduced in appendix B (see, e.g., (B.23) and (B.24)). The continuity at thresholds is a general property of cross sections for a short-range potential. For atomic potentials with a Coulombic asymptotic behaviour the threshold peculiarities of multiphoton cross sections and nonlinear susceptibilities will be more distinct owing to the condensation of Rydberg resonances below each threshold [148] (see, e.g., [149] for numerical examples). It is important to note that all threshold peculiarities are caused by the Fourier coefficients $\phi_{k}$ (given by (B.10)) of the QQES wavefunction at the origin; these peculiarities vanish in the approximation that $\chi_{k} \sim \delta_{k, 0}$ in (56). Moreover, imaginary parts of these coefficients enter the ED terms in ADs, which are proportional to the polarization-angular factor $\sim l \operatorname{Im}\left\{(\boldsymbol{e} \cdot \boldsymbol{n})^{2}\right\}$. Therefore, in contrast to strong field or low frequency (adiabatic) approximations, when usually all but the leading Fourier coefficients of $\Phi_{\epsilon}(\boldsymbol{r}, t)$ at $r \rightarrow 0$ are neglected, in the perturbative regime a proper account of these coefficients is essential in order to describe all major features of multiphoton ADs.

As has also been mentioned above, the total decay rate, $\Gamma=-2 \operatorname{Im} \epsilon$, is independent of an explicit form of the normalized QQES wavefunction and of a definition of the partial rates, $\Gamma^{(n)}$, in terms of wavefunctions ( $\operatorname{cf}(38)$ ). On the other hand, the relation (39) may be used for an independent test of the accuracy of different definitions of the partial rates $\Gamma^{(n)}$ for the case of moderate or strong laser fields, when the LOPT result is insufficient. We illustrate this fact below by comparison of our results for one- and two-photon detachment obtained by use of RS PT with the imaginary part of the two-term expansion (B.14) for the (complex) quasienergy. For $1 / 2<\omega<1$ the one-photon channel is closed; thus the polarizability $\alpha(\omega)$ is real and Im $\gamma_{4}$ in (B.16)-(B.18) determines $\Gamma^{(2)}$, which coincides with the results in (50) or (66). For $\omega>1$, both one- and two-photon channels are open, and thus the self-consistency relation (39)

$$
\frac{1}{2}\left(\operatorname{Im} \alpha(\omega)+\frac{1}{6} \operatorname{Im} \gamma_{4}(\omega ; l) F^{2}\right) F^{2}=\Gamma^{(1)}+\Gamma^{(2)}
$$

should be fulfilled, where $\Gamma^{(2)}$ is defined by equation $(50)$ (or $(65)$, for $l=1$ ) and $\Gamma^{(1)}$ is given by equation (92) below. The explicit expressions for $\alpha$ and $\gamma_{4}$ in (B.15)-(B.18) confirm the validity of the relation (67). Moreover, we have performed a similar comparison for $\omega>1$ of the analytical result for the imaginary part of $\epsilon$ including terms $\sim F^{6}$ with the sum of the total rates (integrated over $\boldsymbol{n}$ ) for one-, two- and three-photon detachment. (For $n=2$ the correction $\sim F^{2}$ to the result (49) has been calculated analytically.) We have observed the complete coincidence of results for these two independent calculations. These analytical considerations justify the self-consistency of both our normalization procedure for the QQES (including the complexity of the normalization parameter $\phi_{0}$ ) and our definition for the differential rates (taking into account the complex 'momentum' $k_{n}$ in (38)). Note that the definition (38) is not conventional, i.e. other authors use definitions that differ from (38) (see, e.g., [125, 126, 150]). Note, however, that in these works, firstly, the normalization factor for the QQES wavefunction appears not to be complex and, secondly, only the real part of the complex quasienergy, $\operatorname{Re} \epsilon$, 
is used in the definition of the 'momentum' $k_{n}$. Therefore, for example, using these definitions for PT calculations of $\Gamma^{(1)}$ and $\Gamma^{(2)}$ even up to $\sim F^{4}$, the self-consistency relation (67) will not be fulfilled.

\section{Angular distributions and elliptic dichroism in the weak field limit}

Multiphoton detachment with elliptically polarized light produces photoelectron ADs that are sensitive to the sign of the ellipticity and that have lower symmetry than those produced with linearly or circularly polarized light. Furthermore, the terms in the AD that are sensitive to the photon helicity result from non-Hermitian components of the multiphoton transition amplitudes or, more precisely, interference between real and imaginary parts of transition amplitudes. Thus, the ED effect provides experimentalists with a means to measure such interference and provides theorists with a sensitive test of theoretical models. The most straightforward way to uncover the origins of these effects is to use the PT approach, which we do here for a general $n$-photon detachment process. The ED effect always stems from the interaction of the detached electron with the atomic core. In the LOPT limit, this interaction originates, first, from ED terms in $n$-photon ADs that involve nonzero detached electron phase shifts (which describe the elastic scattering of the detached electron from the atomic core). For the case of ATD, it originates also from additional ED terms involving the amplitudes of inelastic electron-core scattering after absorption of $m$ photons $((1 / \omega)<m<n)$ by an escaping electron. We discuss the quantitative contributions of these alternative ED terms for the case of the $\delta$-model potential. We also present explicit AD results for $2 \leqslant n \leqslant 8$ and demonstrate a good agreement of these predictions based upon the $\delta$-model potential with available experimental data and with results of more elaborate calculations for the $\mathrm{H}^{-}$ion.

\subsection{Perturbative analysis of angular distributions for elliptically polarized light}

For a circularly polarized laser field, the LOPT results for the differential and total rates have simple forms for absorption of $n$ photons:

$$
\Gamma_{c i r c}^{(n)}(n)=\frac{\Gamma_{\text {circ }}^{(n)}}{4 \pi} \frac{(2 n+1) ! !}{(2 n) ! !}(\sin \theta)^{2 n}, \quad \Gamma_{\text {circ }}^{(n)}=\frac{2^{n+2}}{(2 n+1) !}\left(\frac{F}{\omega^{2}}\right)^{2 n}(n \omega-1)^{n+\frac{1}{2}} .
$$

These results follow immediately from (57) and (58), where only terms with $k=0$ contribute for $^{10} l=0$. Here $\theta$ is the angle between $n$ and $\hat{k}$. With the use of the Clebsch-Gordan expansion for the product of Legendre polynomials, one may evaluate the squared modulus of the amplitude in (61) to obtain explicit analytic expressions for the AD asymmetry parameters, $\beta_{2 k}(\omega)$, for the case of a linearly polarized field ${ }^{11}$ :

$$
\Gamma_{l i n}^{(n)}(\boldsymbol{n})=\frac{\Gamma_{l i n}^{(n)}}{4 \pi}\left[1+\sum_{k=1}^{n} \beta_{2 k}^{(n)} P_{2 k}(\cos \alpha)\right]
$$

where

$\beta_{2 k}^{(n)}(\omega)\left(\sum_{L=L_{\min }}^{n}\left|a_{L}(\omega)\right|^{2}\right)=\sum_{L_{1}, L_{2}=L_{\min }}^{n}\left(C_{L_{1} 0 L_{2} 0}^{2 k 0}\right)^{2} \sqrt{\left(2 L_{1}+1\right)\left(2 L_{2}+1\right)} a_{L_{1}}(\omega) a_{L_{2}}^{*}(\omega)$

and where $a_{L}(\omega)$ and $\Gamma_{\text {lin }}^{(n)}$ are given by equations (61)-(64).

10 The energy dependence in (68) follows obviously from Wigner's law (B.25).

${ }^{11}$ Note that the general form of AD in (69) is valid for any momentumless target ('Yang's theorem') [152]. 
For an elliptic polarization, the formulae for the ADs are much more involved. Besides the loss of axial symmetry (with the symmetry axis being along $\hat{\epsilon}$ for $\xi=0$ ) or along $\hat{k}$ for $l=0$ ) a dependence on the sign of $\xi$ appears for $0<|\xi|<1$. In [11, 49] it was shown that this dependence is due to the term $l \operatorname{Im}\left\{(e \cdot n)^{2}\right\}$ which occurs in both the lowest- and higher-order PT results for the ADs. General symmetry arguments as well as our explicit results for $2 \leqslant n \leqslant 5$ in section 2.6 and our general result for arbitrary $n$ in (57) show that the polarization-angular dependence of the $n$-photon $\operatorname{AD}^{(n)}(e ; n)$ for an arbitrary ellipticity may be presented as the sum of combinations of the linear polarization degree, $l$, and two fundamental vector constructions, $|\boldsymbol{e} \cdot \boldsymbol{n}|^{2}$ and $l(\boldsymbol{e} \cdot \boldsymbol{n})^{2}$, whose representations in terms of real vectors (cf the definition (4) and parametrizations (6) and (7) in terms of spherical angles $\theta$ and $\varphi)$ are [49]

$$
\begin{aligned}
& 2|\boldsymbol{e} \cdot \boldsymbol{n}|^{2}=(1+l)(\hat{\epsilon} \cdot \boldsymbol{n})^{2}+(1-l)(\boldsymbol{n} \cdot[\hat{k} \times \hat{\epsilon}])^{2}, \\
& 2 \operatorname{Re}\left\{(\boldsymbol{e} \cdot \boldsymbol{n})^{2}\right\}=(1+l)(\hat{\epsilon} \cdot \boldsymbol{n})^{2}-(1-l)(\boldsymbol{n} \cdot[\hat{k} \times \hat{\epsilon}])^{2}, \\
& \operatorname{Im}\left\{(\boldsymbol{e} \cdot \boldsymbol{n})^{2}\right\}=\xi(\hat{\epsilon} \cdot \boldsymbol{n})(\boldsymbol{n} \cdot[\hat{k} \times \hat{\epsilon}]) .
\end{aligned}
$$

An invariant four-parameter representation of the two-photon AD is

$$
\frac{1}{I^{2}} \Gamma^{(2)}(\boldsymbol{e} ; \boldsymbol{n})=l^{2} A_{1}^{(2)}+A_{2}^{(2)}|\boldsymbol{e} \cdot \boldsymbol{n}|^{4}+A_{3}^{(2)} l \operatorname{Re}\left\{(\boldsymbol{e} \cdot \boldsymbol{n})^{2}\right\}+A_{4}^{(2)} l \operatorname{Im}\left\{(\boldsymbol{e} \cdot \boldsymbol{n})^{2}\right\},
$$

where the explicit forms for the factors $A_{i}^{(2)}$ are evident by comparison of (72) with (49). Equation (72) coincides with the general form of the AD for two-photon ionization of atoms for the case of an initial S-state [49]. The result (52) for $n=3$ may be presented in the same form as (72) with an additional overall factor $|e \cdot n|^{2}$ on the rhs. In both cases only one parameter, $A_{2}^{(i)}$, contributes for $l=0$. For $l=1$ only the term with the ED parameter $A_{4}^{(i)}$ vanishes. Note that for $l=1$ the parameters $A_{1-3}^{(i)}$ are simply related to the asymmetry parameters $\beta_{2 k}^{(i)}$ in (69). In particular, for $n=2$ we have

$$
\beta_{2}^{(2)}=\frac{2}{3 \Sigma^{(2)}}\left(\frac{6}{7} A_{2}^{(2)}+A_{3}^{(2)}\right), \quad \beta_{4}^{(2)}=\frac{8}{35 \Sigma^{(2)}} A_{2}^{(2)},
$$

where $\Sigma^{(2)}=A_{1}^{(2)}+(1 / 5) A_{2}^{(2)}+(1 / 3) A_{3}^{(2)}$ and $\Gamma_{\text {lin }}^{(2)}=4 \pi I^{2} \Sigma^{(2)}$ is the total two-photon rate for the case of linear polarization. Similar results for $n=3$ in (70) are

$$
\begin{array}{ll}
\Sigma^{(3)}=\frac{1}{3} A_{1}^{(3)}+\frac{1}{7} A_{2}^{(3)}+\frac{1}{5} A_{3}^{(3)}, & \beta_{2}^{(3)}=\frac{2}{3 \Sigma^{(3)}}\left(A_{1}^{(3)}+\frac{5}{7} A_{2}^{(3)}+\frac{6}{7} A_{3}^{(3)}\right), \\
\beta_{4}^{(3)}=\frac{8}{35 \Sigma^{(3)}}\left(\frac{15}{11} A_{2}^{(3)}+A_{3}^{(3)}\right), & \beta_{6}^{(3)}=\frac{16}{231 \Sigma^{(3)}} A_{2}^{(3)},
\end{array}
$$

where in this case $\Gamma_{\text {lin }}^{(3)}=4 \pi I^{3} \Sigma^{(3)}$. With increasing $n$, the number of parameters $A_{n}^{(i)}$ grows rapidly. Moreover, the coefficients $\chi_{k}(\omega)$ in (56) are complex (with the exception of $\left.\chi_{0}(\omega)=1\right)$; thus, for $n=4$ and 5 the ADs involve nine parameters including three different dichroic terms $\sim l \xi$. Besides $l^{3} \operatorname{Im}\left\{(\boldsymbol{e} \cdot \boldsymbol{n})^{2}\right\}$ and $l^{2}|\boldsymbol{e} \cdot \boldsymbol{n}|^{2} \operatorname{Im}\left\{(\boldsymbol{e} \cdot \boldsymbol{n})^{2}\right\}$, these terms involve a new construction, $l^{2} \operatorname{Im}\left\{(\boldsymbol{e} \cdot \boldsymbol{n})^{4}\right\}$. In the general case, the $\operatorname{AD} \Gamma^{(n)}(\boldsymbol{e} ; \boldsymbol{n})$ with $n=2 m$ and $n=2 m+1$ involves $(m+1)^{2}$ independent polarization-angular terms, and $m(m+1) / 2$ among them are responsible for the ED effect (i.e. are proportional to the product $l \xi$ ).

In the geometry of figure 1 we have $(\boldsymbol{n} \cdot \hat{\boldsymbol{\epsilon}})^{2}=\cos ^{2} \alpha,(\boldsymbol{n} \cdot[\hat{k} \times \hat{\epsilon}])^{2}=\cos ^{2} \beta$, and thus

$$
l \operatorname{Im}\left\{(\boldsymbol{e} \cdot \boldsymbol{n})^{2}\right\}=\frac{1}{2} \xi l \sin ^{2} \theta \sin 2 \varphi=\xi l \cos \alpha \cos \beta .
$$

Therefore, for the case of an elliptical polarization, the ADs are described by two independent angles (e.g. by $\alpha$ and $\beta$ ) and they are symmetric with respect to the inversion $\boldsymbol{n} \rightarrow-\boldsymbol{n}$, or equivalently, to the simultaneous change $\alpha \rightarrow \alpha+\pi$ and $\beta \rightarrow \beta+\pi$. This symmetry originates 
from conservation of spatial parity and is independent of the polarization state. Note that, if one neglects the dichroic terms, an additional symmetry appears with respect to a rotation by an angle $\pi$ around either the major or the minor axes of the polarization ellipse, i.e. for $\beta \rightarrow \beta+\pi$ or $\alpha \rightarrow \alpha+\pi$. The ED terms destroy this symmetry. In view of the ED, the ADs for opposite helicities of a laser beam, i.e. $\xi$ and $-\xi$, are different and cannot be made to coincide as the result of spatial rotations. Nevertheless, a symmetry exists with respect to the substitution $\xi, \hat{k} \rightarrow-\xi,-\hat{k}$ (see (71)). In other words, the ADs in two experiments with opposite helicities and directions of the laser beam will be the same. Equation (75) shows that this latter symmetry corresponds to a simultaneous change $\xi \rightarrow-\xi$ and either $\alpha \rightarrow \alpha+\pi$ or $\beta \rightarrow \beta+\pi$. Obviously, measurements of dichroic terms in ADs would provide information on the laseratom interaction and on the atomic parameters that is inaccessible to experiments that employ linearly or circularly polarized light sources. As examples of both the variation of ADs when the ellipticity varies and the asymmetry caused by the ED terms, we present in figures 3-9 (unnormalized) results for three-dimensional ADs for $n \leqslant 8$.

Figures 3(a)-(e) and 4(a)-(d) show ADs for two- and three-photon detachment of $\mathrm{H}^{-}$at the frequency $\omega=0.862$ that has been used in a recent experiment on two-photon detachment of $\mathrm{H}^{-}$by a linearly polarized laser field [68]. Our results in figures 3 and 4 are presented for $\xi=0,0.7,-0.7$ and for $n=2, \xi= \pm 1$. For circular polarization $(\xi= \pm 1)$ the ADs have a high symmetry and are similar for any $n$ (see (68)); thus we present results for this case only for $n=2$, in figure 3(d). In figures 3(e) and 4(d) we present two-dimensional ADs in the plane of the laser polarization, i.e. in the plane of the vectors $\hat{\epsilon}$ and $[\hat{k} \times \hat{\epsilon}]$. One sees that for both $n=2$ and 3 the ED effects are significant enough to be measured experimentally. Note that for any fixed frequency only four measured data points are sufficient to determine the total set of four invariant atomic parameters $A^{(i)}$ for two- or three-photon detachment in the perturbative regime. These data points may be, e.g., the total detachment yield or two measurements for some fixed geometry but for opposite helicities of the laser field, and an additional measurement for one other value of the angles $\alpha$ and/or $\beta$. With these four data points in hand, our analytical results allow one to reconstruct the entire (normalized) three-dimensional AD for any geometry and polarization state of the laser field.

Two-dimensional ADs for two- and three-photon detachment of $\mathrm{H}^{-}$in an elliptically polarized field have been analysed in [77] using $B$-spline calculations that treat electron correlations. For the case of orthogonal geometry, i.e. $\theta=\pi / 2$, our analytical results for two- and three-photon amplitudes coincide with those given in terms of radial matrix elements that are presented in the appendix of [77]. We found also good agreement with the $B$-spline calculations [77] for the ellipticity dependences of the total rates for $n=2$ and 3 (cf our figure 12(b) for $n=2$ with figure 4 of [77]). Nevertheless our results for ADs in the plane of the polarization ellipse disagree with the results of [77], both for $n=2$ and 3. For example, in figure 5 we present our planar AD results for $n=3$ and for a number of values of $\eta$ used in [77]. These results differ substantially from the corresponding results in figure 8 of [77], both for below- (figure 5(a)) and above-threshold frequencies (figure 5(b)). Since in accordance with (73) the parameters $A_{i}^{(2)}$ with $i=1,2,3$, are directly connected with the asymmetry parameters and with the total rate, for which our results are in reasonable agreement with other accurate calculations (see section 3.3), it may be expected that differences in the values of the ED parameter, $A_{4}^{(2)}$, obtained in the $\delta$-model and in the $B$-spline calculations can explain the observed disagreement in the ADs for $n=2$. Consequently, we varied this arbitrary parameter over a range of $\sim( \pm 50) \%$, but we were still unable to obtain any agreement of these results with those of [77]. Thus, the reason for this disagreement is unclear to us. An independent calculation would thus be very interesting and appears necessary to resolve this contradiction. 

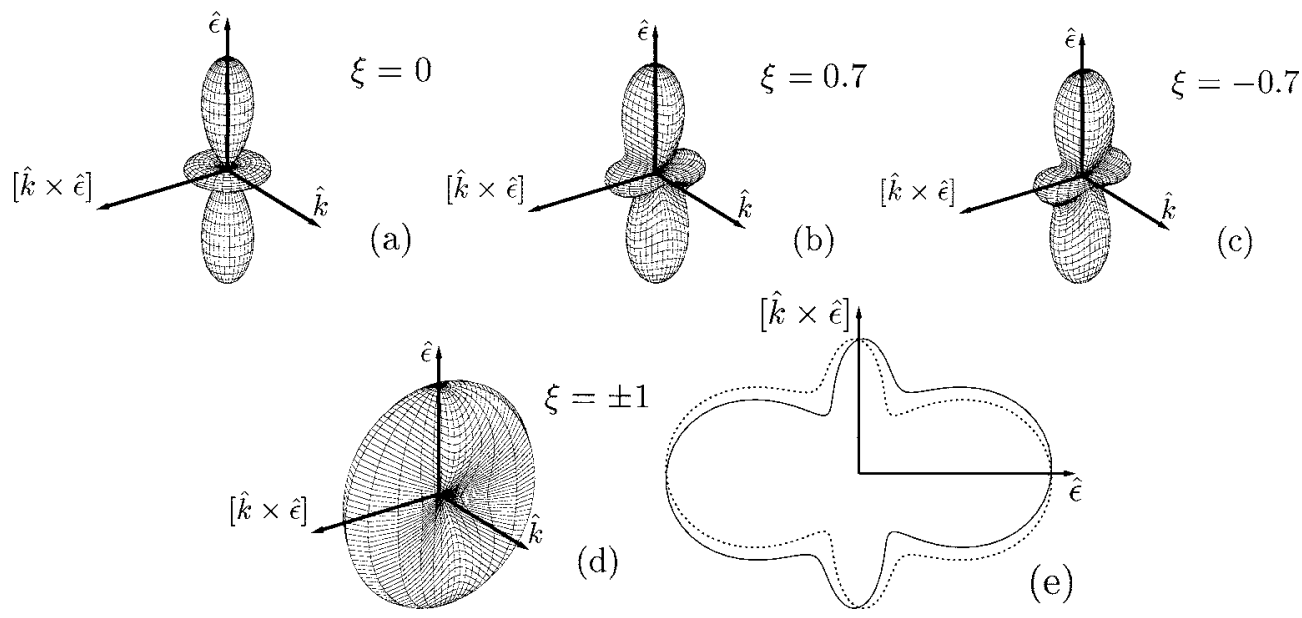

Figure 3. The $\mathrm{AD}$ of detached electrons for two-photon detachment of $\mathrm{H}^{-}$for $\omega=0.862$ and (a) $\xi=0$, (b) $\xi=0.7$, (c) $\xi=-0.4$ and (d) $\xi= \pm 1$. (e) Two-dimensional ADs of detached electrons for two-photon detachment of $\mathrm{H}^{-}$in the polarization plane defined by $\hat{\epsilon}$ and $[\hat{k} \times \hat{\epsilon}]$ for $\omega=0.862$ and for two different ellipticities, $\eta$. Full curve: $\xi=0.7(\eta=0.4)$; dotted curve: $\xi=-0.7(\eta=-0.4)$. The differences of the two curves arise from the ED effect.

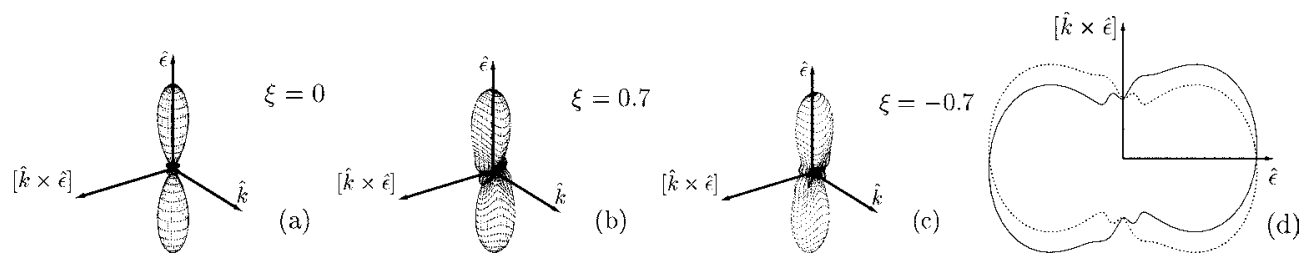

Figure 4. The same as figure 3 for three-photon ATD of $\mathrm{H}^{-}$.
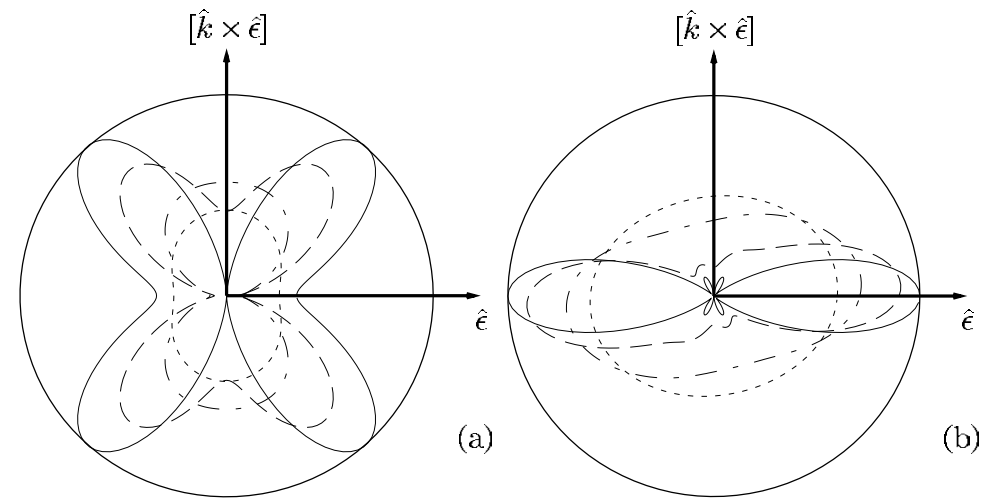

Figure 5. Two-dimensional ADs for three-photon detachment of $\mathrm{H}^{-}$for various ellipticities (full curve: $\xi=0(\eta=0)$; broken curve: $\xi=0.64(\eta=0.36)$; chain curve: $\xi=0.94(\eta=0.7)$; dotted curve: $\xi=0.99(\eta=0.9))$ and for the same two values of the photoelectron energy, $E_{p}=\omega-1$, as in figure 8 of [77]. (a) $\omega=0.4055$ ( $\left.E_{p}=0.006 \mathrm{au}\right)$; (b) $\omega=0.76655$ ( $E_{p}=0.036 \mathrm{au}$ ).

The ADs for above-threshold four-photon $\left(\omega=0.31 \simeq 2 \omega_{\mathrm{CO}_{2}}\right)$ and five-photon $(\omega=0.39)$ detachment are presented in figures 6(a)-(d) and 7(a)-(d). Because of the small 


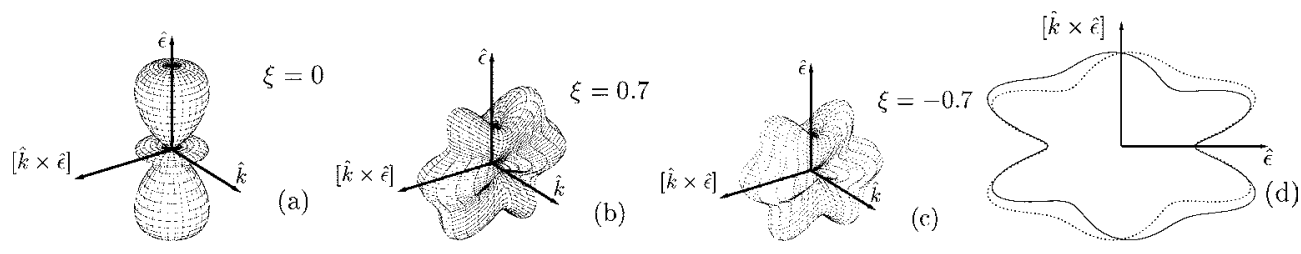

Figure 6. The $\mathrm{AD}$ of detached electrons for four-photon detachment of $\mathrm{H}^{-}$for $\omega=0.31$ and (a) $\xi=0$, (b) $\xi=0.7$, (c) $\xi=-0.7$. (d) Two-dimensional AD of detached electrons and ED for four-photon detachment of $\mathrm{H}^{-}$(at $\omega=0.31$ ) in the polarization plane defined by $\hat{\epsilon}$ and $[\hat{k} \times \hat{\epsilon}]$. Full curve: $\xi=0.7$; dotted curve: $\xi=-0.7$.

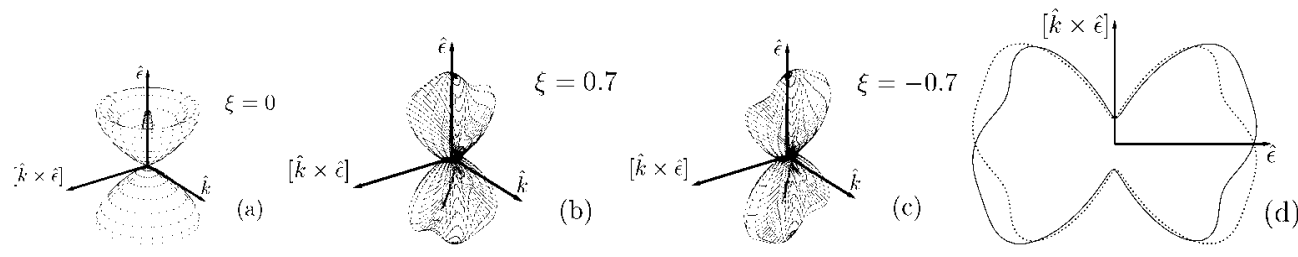

Figure 7. The AD of detached electrons for five-photon ATD of $\mathrm{H}^{-}$for $\omega=0.39$ and (a) $\xi=0$, (b) $\xi=0.7$, (c) $\xi=-0.7$. (d) Two-dimensional AD of detached electrons and $\mathrm{ED}$ for five-photon detachment of $\mathrm{H}^{-}$(at $\omega=0.39$ ) in the polarization plane defined by $\hat{\epsilon}$ and $[\hat{k} \times \hat{\epsilon}]$. Full curve: $\xi=0.7$; dotted curve: $\xi=-0.7$.
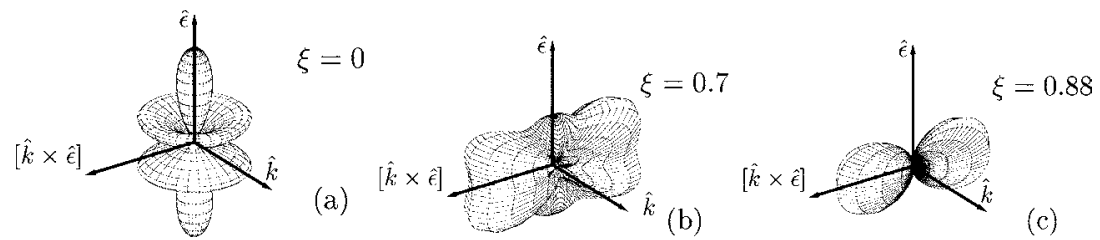

Figure 8. The $\mathrm{AD}$ of detached electrons for seven-photon detachment of $\mathrm{H}^{-}$for $\omega=0.15504$ $\left(\mathrm{CO}_{2}\right.$ laser) and (a) $\xi=0$, (b) $\xi=0.7$, (c) $\xi=0.88$.

binding energy of $\mathrm{H}^{-}$, it seems that an analysis of detachment for high photon numbers $n$ for the case of 'below-threshold' frequencies, $1 / n<\omega<1 /(n-1)$, is not interesting for experimental measurements, except for the important case of the $\mathrm{CO}_{2}$ laser, whose wavelength of $10.6 \mu \mathrm{m}$ corresponds to seven-photon detachment. The results for this case $\left(\omega_{\mathrm{CO}_{2}}=0.15504\right)$ are presented in figures 8 (a)-(c) for $\xi=0,0.7$ and 0.88 . Since for odd $n$ the ED asymmetry vanishes for 'below-threshold' frequencies (see below), the ADs in figures 8(b) and (c) are independent of the sign $\xi$ and are symmetric with respect to the substitutions $\alpha \rightarrow \alpha+\pi$ or $\beta \rightarrow \beta+\pi$. Moreover, although for this case the ADs depend only on $\xi^{2}$, their form depends significantly on the absolute value of $\xi$. In figures 9(a)-(d) results for eight-photon ATD for the $\mathrm{CO}_{2}$ frequency are presented. Here the ED asymmetry is displayed very clearly. For a crude estimation of the intensity at which the seven- and eight-photon cross sections are comparable and for which the ED effect may be visible, we mention that the photoelectron yield for $n=8$ constitutes $10 \%$ of that for $n=7$ at a critical intensity, $I_{c r} \simeq 10^{8} \mathrm{~W} \mathrm{~cm}^{-2}$, for the $\mathrm{H}^{-}$ion (for the case of fixed $\xi=0.7$ and $\alpha=\beta=\pi / 4$ ). The magnitude of $I_{c r}$ grows rapidly with increasing $\omega$ in the above-threshold domain; for instance, $I_{c r} \simeq 1.4 \times 10^{11} \mathrm{~W} \mathrm{~cm}^{-2}$ for $\omega=0.55$. 

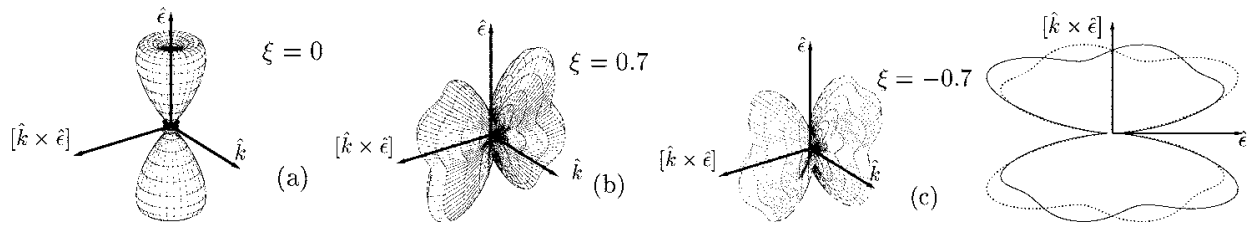

Figure 9. The AD of detached electrons for eight-photon ATD of $\mathrm{H}^{-}$for $\omega=0.15504\left(\mathrm{CO}_{2}\right.$ laser $)$ and (a) $\xi=0$, (b) $\xi=0.7$, (c) $\xi=-0.7$. (d) Two-dimensional AD in the polarization plane defined by $\hat{\epsilon}$ and $[\hat{k} \times \hat{\epsilon}]$. Full curve: $\xi=0.7$; dotted curve: $\xi=-0.7$.
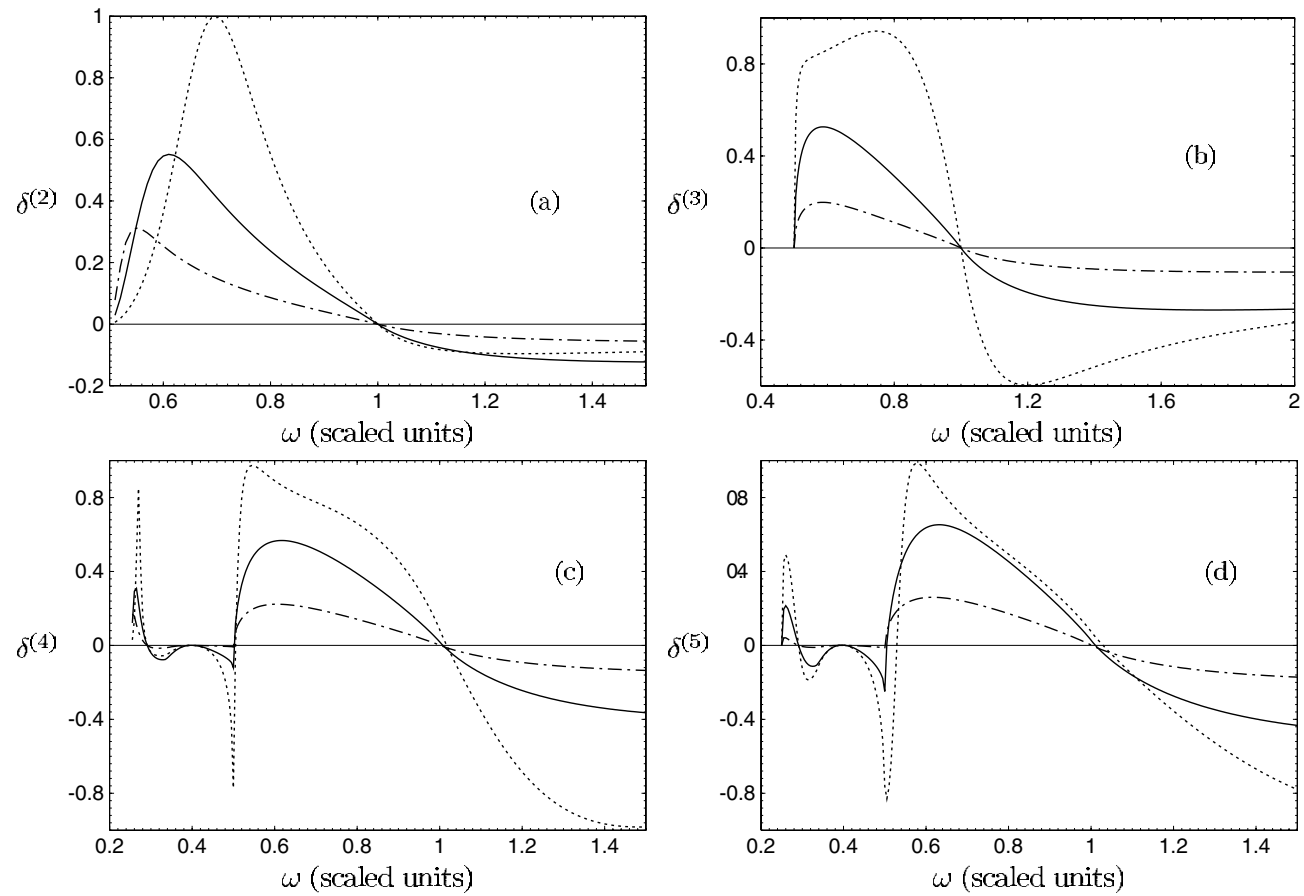

Figure 10. Frequency dependence of the relative ED parameter, $\delta^{(n)}$ (see (77)), for $n$-photon detachment of $\mathrm{H}^{-}$in the LOPT: (a) $\delta^{(2)}$; (b) $\delta^{(3)}$; (c) $\delta^{(4)}$; (d) $\delta^{(5)}$. The geometric parameters are: $\theta=\pi / 2 ; \alpha=\beta=\pi / 4$; the polarization parameters are: dotted curve: $\xi=0.2$; full curve: $\xi=0.7$; chain curve: $\xi=0.94$.

\subsection{Discussion of dichroic terms in ADs}

We discuss now in more detail the magnitude of the ED effect and its frequency dependence. Consider first the cases of $n=2$ and 3 based on (49) and (52). For $n=2$ the polarization and angular dependence of the ED effect is rather simple:

$$
\Delta_{e d}^{(2)} \equiv \Gamma^{(2)}(\xi ; \boldsymbol{n})-\Gamma^{(2)}(-\xi ; \boldsymbol{n})=I^{2} \beta_{e d}^{(2)} l \xi \cos \alpha \cos \beta
$$

The kinematical maximum of the ED term corresponds to $\alpha=\beta=\pi / 4, \theta=\pi / 2$, and $\xi^{2}=l^{2}=0.5$. In figure 10(a) we present the frequency dependence of the dimensionless ED parameter, $\delta^{(2)}$, where

$$
\delta^{(n)}=\Delta_{e d}^{(n)}\left[\Gamma^{(n)}(\xi ; \boldsymbol{n})+\Gamma^{(n)}(-\xi ; \boldsymbol{n})\right]^{-1}
$$


for $\mathrm{H}^{-}, \theta=\pi / 2$ and $\alpha=\beta=\pi / 4$. For $n=2$ and 3, the ED term $\Delta_{e d}^{(n)}$ has in general the same magnitude as other terms in the cross sections, so that $\delta^{(n)}$ may attain values of the order of unity. The angular dependence of $\Delta_{e d}^{(3)}$ differs from that in (76):

$\Delta_{e d}^{(3)} \equiv \Gamma^{(3)}(\xi ; \boldsymbol{n})-\Gamma^{(3)}(-\xi ; \boldsymbol{n})=\beta_{e d}^{(3)} l \xi \cos \alpha \cos \beta\left[(1+l) \cos ^{2} \alpha+(1-l) \cos ^{2} \beta\right] I^{3}$.

In this case the rhs of (78) has a maximum at $\theta=\pi / 2, \xi=2 / 3$ and $\tan (2 \alpha)=2$. The frequency dependence of $\delta^{(3)}$ is presented in figure 10(b).

As shown in figures 10(a) and (b), the parameters $\delta^{(n)}$ for $n=2$ and 3 vanish at thresholds and change sign after the opening of the $n=1$ channel [78]. The ED effect in two-photon detachment is most significant for below-threshold frequencies; it is small for $\omega>1$, when the one-photon channel is open. On the contrary, for $n=3$ the ED effect is nonzero only for above-threshold frequencies, $\omega>1 / 2 ; \delta^{(3)}$ achieves considerable values above both the twophoton and the one-photon thresholds. These peculiarities are transparent from the explicit formulae for $\beta_{e d}^{(n)}$ which easily follow from (49) and (52). In all cases $\beta_{e d}^{(n)}$ originates from an interference between real and imaginary parts of various terms which enter the detachment amplitude. In particular,

$$
\begin{aligned}
\beta_{e d}^{(2)} \equiv A_{4}^{(2)}= & \frac{1}{3 \pi \omega^{9}}(2 \omega-1)^{2}(1-\omega) \\
& \times\left[\Theta(1-\omega)(1-\sqrt{1-\omega})+\Theta(\omega-1)\left(1-\sqrt{\frac{\omega-1}{2 \omega-1}}\right)\right],
\end{aligned}
$$

where the Heaviside function $\Theta(x)$, is unity for $x \geqslant 0$ and is zero for $x<0$. The parameter $\beta_{e d}^{(3)}$ is very similar to $\beta_{e d}^{(2)}$ :

$$
\begin{aligned}
\beta_{e d}^{(3)} \equiv A_{4}^{(3)}= & \frac{1}{9 \pi \omega^{13}}(3 \omega-1)^{5 / 2} \sqrt{2 \omega-1}(1-\omega) \Theta(2 \omega-1) \\
& \times\left[\Theta(1-\omega)(1-\sqrt{1-\omega})+\Theta(\omega-1)\left(1-\sqrt{\frac{\omega-1}{2 \omega-1}}\right)\right] .
\end{aligned}
$$

The first term on the rhs of equation (79) for $\beta_{e d}^{(2)}$ corresponds to the case $\omega<1$ while the second one contributes only when ATD takes place. Moreover, $\beta_{e d}^{(3)}$ in (80) is zero in the belowthreshold domain, $1 / 3<\omega<1 / 2$. The physical meaning of these results becomes more clear by re-writing $\beta_{e d}^{(n)}$ in terms of $n$ th-order PT radial matrix elements, $M_{L}^{(n)}$, and scattering phases, $\delta_{L}(E)$, where $L$ is the angular momentum of the detached electron. In particular, the result (79) in terms of these quantities is [49]

$$
\begin{aligned}
\beta_{e d}^{(2)}=-\frac{1}{24 \pi^{2} \omega} & \sqrt{2 \omega-1} M_{2}\left[\Theta(1-\omega) \sin \delta_{0} M_{0}+\Theta(\omega-1)\right. \\
\times & \left.\left\{\sin \delta_{0} \operatorname{Re} M_{0}+\cos \delta_{0} \operatorname{Im} M_{0}\right\}\right],
\end{aligned}
$$

where $\delta_{0} \equiv \delta_{0}(2 \omega-1)$. Note that, for a $\delta$-model potential, $\delta_{L>0}=0$ and $\exp \left(\mathrm{i} \delta_{0}\right)=$ $(1-\mathrm{i} \sqrt{2 \omega-1}) / \sqrt{2 \omega}$ (cf $\left(\right.$ B.21)) and the matrix elements $M_{L}^{(n)}$ with $L=n$ are real. Equation (81) shows that for $\omega<1$ the ED term is proportional to $\sin \delta_{0}$ (note that it is this scattering phase which causes the complexity of the coefficient $g_{1}(\omega)$ in (B.23) at $1 / 2<\omega<1$ ), while in the above-threshold case the ED term involves $\operatorname{Im} M_{0}$. The imaginary part of $M_{0}$ corresponds to the amplitude of a two-step (cascade) process, namely one-photon detachment followed by subsequent inelastic scattering of the detached electron by the parent atom with the absorption of one additional photon. This two-step process interferes with the 'direct' two-photon detachment in another $L$ channel, whose amplitude is proportional to 
$\operatorname{Re} M_{2}$, and this interference leads to an ED effect even if the scattering phase $\delta_{0}(2 \omega-1)$ is neglected (which in our formalism corresponds to keeping only the phase $\delta_{0}(\omega-1)$ in (B.23)). This mechanism explains the origin of ED in three-photon detachment: for this case $L=1$ and 3 , and thus $\delta_{L}=0$ so that the ED effect is nonzero only for above-threshold frequencies (see figure 10(b)), at which the radial matrix elements $M_{1}^{(3)}$ are complex. (This corresponds to the complexity of the function $g_{1}(\omega)$ in (B.23) for $\omega>1 / 2$, which enters our result for the amplitude $\mathcal{A}_{3}$.) Since ED in three-photon detachment is caused only by the interference between real and imaginary parts of radial amplitudes, it provides a possibility for the direct measurement of this interference. On the other hand, since in reality the phase $\delta_{1}$ is nonzero (although small), the precise measurement of ED terms (with a relative accuracy of order $\delta_{1}$ ) for below-threshold frequencies allows one to measure this small phase.

The general features of the ED parameters for $n \geqslant 4$ are different from those for the cases $n=2$ and 3 in two respects. First, now the dichroic difference, $\Delta_{e d}^{(n)}$, has a more complicated form involving a number of terms with different dependences on the angles $\alpha$ and $\beta$, as may be seen from the general result for the amplitude given in (56). In particular, for $n=4$ the result for $\Delta_{e d}^{(n)}$ may be written as

$$
\Delta_{e d}^{(4)}=I^{4} l \xi \cos \alpha \cos \beta a_{1}^{(4)}\left|a_{2}^{(4)} l+a_{3}^{(4)}(e \cdot n)^{2}\right|^{2},
$$

where the frequency-dependent factors $a_{i}^{(4)}$ are real and may be easily obtained from (54). Similarly, $\Delta_{e d}^{(5)}$ is given by (82) with an overall factor $I|(e \cdot n)|^{2}$ on the rhs and other invariant parameters $a_{i}^{(5)}$ (see (54)). Obviously, in all cases the ED parameters such as $a_{i}^{(n)}$ arise from an interference between real and imaginary parts of the factors $\chi_{k}(\omega)$ in the detachment amplitude (56) (or, equivalently, of the Fourier coefficients of the QQES wavefunction at the origin). Therefore, the ED effect vanishes if one neglects the interaction of the escaping electron with the binding potential, as is done in the KA and also in various 'SFAs' that are similar to it; it vanishes also if this interaction is treated in a crude (e.g. Born) approximation in which the transition amplitude is Hermitian and independent of the scattering phases. Furthermore, for $n \geqslant 4$ many coefficients $\chi_{k}(\omega)$ contribute to the requisite interference and thus the frequency dependence of the ED terms is not as regular as for the cases $n=2$ and 3 (cf (79) and (80)), in which only one coefficient, $\phi_{1}(\omega)$, enters the amplitudes $\mathcal{A}_{2}$ and $\mathcal{A}_{3}$. A consequence of this fact is that the relative ED parameter $\delta^{(n)}$, (77), does not vanish at threshold frequencies (although it changes its sign in a near-threshold domain). Of course, the above-discussed statement on the vanishing of ED for $n=3$ for 'below-threshold' frequencies (owing to the vanishing of the scattering phases $\delta_{L}$ with $L>0$ for a short-range potential) is valid for any odd $n=2 k+1$ in the limit of LOPT, as may be confirmed by a direct analysis based on the explicit result (56) for $\mathcal{A}_{n}$.

As an illustration of the above statements, in figures $10(\mathrm{c})$ and (d) we present the frequency dependence of $\delta^{(n)}$ for $n=4$ and 5 for the orthogonal geometry, $\boldsymbol{n} \cdot \boldsymbol{k}=0$, and $\alpha=\beta=\pi / 4$. In addition, in figure 11 we plot $\delta^{(n)}$ (for $n=2,3,4,5$ ) as a function of $1 / \omega$. The inverse frequency dependence clearly separates the $n$-photon thresholds and shows very clearly that both the three- and five-photon ADs do not have any asymmetry for $\xi \rightarrow-\xi$ over the ranges $2<\omega^{-1}<3$ and $4<\omega^{-1}<5$, respectively. Since we are in the PT regime, for any range of the frequency, the rate with the lowest allowed number of absorbed photons determines almost entirely the total detachment rate. Therefore, we expect to see flat (i.e. zero) regions for $2 p<\omega^{-1}<2 p+1$ for the total ED parameter, $\delta^{(t o t a l)}$, which is defined by replacing in $(77) \Gamma^{(n)}(\xi ; \boldsymbol{n})$ by the total $\mathrm{AD}$ rate, $\Gamma(\xi ; \boldsymbol{n})=\sum_{n} \Gamma^{(n)}(\xi ; \boldsymbol{n})$. These flat (zero) regions are clearly visible in figure 11 .

In summary, all the major properties of the ADs and $n$-photon rates, $\Gamma^{(n)}$, in the PT approach are caused by the functions $\mathcal{D}_{k}(E+p \omega)$ in (B.2) (or, in LOPT, by $\chi_{k}(\omega)$ from (B.11) 

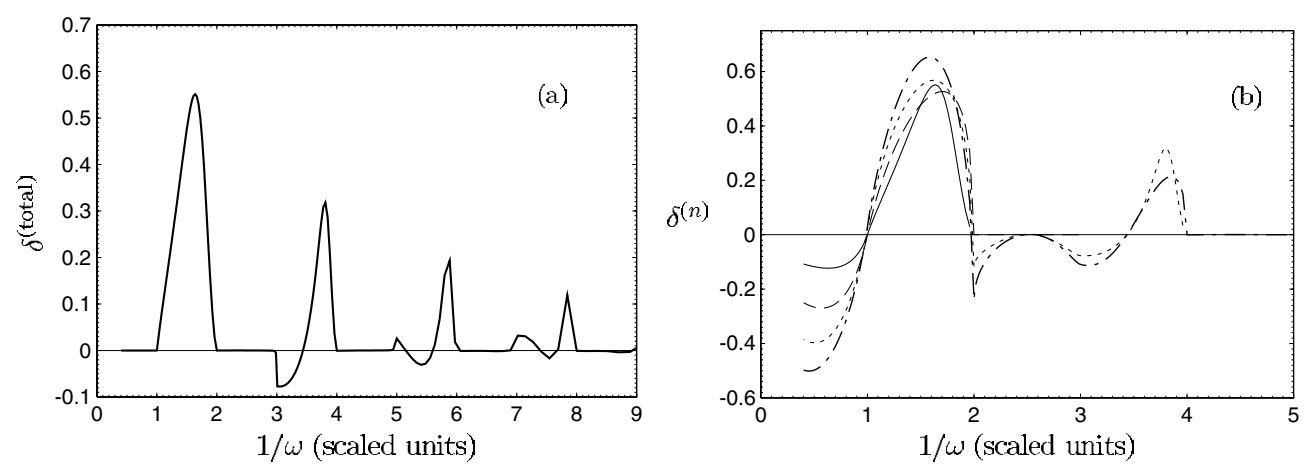

Figure 11. (a) The total ED parameter. (b) The two-, three-, four- and five-photon ED parameters, $\delta^{(n)}$, as functions of $1 / \omega$. Full curve: $\delta^{(2)}$; broken curve: $\delta^{(3)}$; dotted curve: $\delta^{(4)}$; chain curve: $\delta^{(5)}$. The ellipticity is $\xi=0.7$ and $F=0.01$.

and (B.12)). Physically, the dichroic effects originate from an interaction of the detaching electron with an atomic potential $U(\boldsymbol{r})$ both in intermediate (after absorption of $m<n$ photons) and final states. In the $\delta$-model potential, this coupling of the detaching electron with a core potential is realized through the $S$-wave scattering phase. Thus, neglect of this phase (see, e.g., an analysis of $n$-photon detachment in a linearly polarized field in this approximation in [86] or similar calculations for an elliptic polarization in the appendix of paper [77]), whose contribution may seem negligible at first sight, leads to results that, like those for the case of circular polarization, have none of the phase-dependent interference terms that are inherent to (and which characterize) the ADs produced by a laser field having an elliptic (or linear) polarization.

\subsection{Comparisons with recent experiments and multielectron calculations}

Recent experiments $[67,68]$ have measured ADs in two-photon detachment of $\mathrm{H}^{-}$by a linearly polarized laser field. For linear polarization, the ADs are completely described by asymmetry parameters $\beta_{2 k}^{(n)}$, see (69) and (70). Thus, for $n=2$ we have

$$
\Gamma_{\text {lin }}^{(2), \mathrm{H}^{-}}(\boldsymbol{n})=\frac{\Gamma_{\text {lin }}^{(2), \mathrm{H}^{-}}}{4 \pi}\left[1+\beta_{2} P_{2}(\cos \alpha)+\beta_{4} P_{4}(\cos \alpha)\right],
$$

where $\alpha$ is the angle between $\boldsymbol{n}$ and the direction of the linear polarization, $\hat{\epsilon}=e$. The total rate $\Gamma_{\text {lin }}^{(2)}$ is given by (50) with $\xi=0, l=1$. The simple analytic results for the parameters $\beta_{2} \equiv \beta_{2}^{(2)}$ and $\beta_{4} \equiv \beta_{4}^{(2)}$ easily follow from equations (49), (72) and (73):

$$
\begin{aligned}
& \beta_{2}=\frac{10(2 \omega-1)}{7}\left[\frac{4 \omega^{2}+5 \omega-7+7|\omega-1|^{3 / 2}\left(\begin{array}{c}
1, \quad 1 / 2<\omega<1 \\
\left.\sqrt{2 \omega-1}, \quad \begin{array}{c}
1 \\
\omega
\end{array}\right)
\end{array}\right],}{2(2 \omega-1)^{2} \omega+5(\omega-1)^{2}|1+i \sqrt{\omega-1}|^{2}}\right] \\
& \beta_{4}=\frac{36(2 \omega-1)^{2} \omega}{7\left(2(2 \omega-1)^{2} \omega+5(\omega-1)^{2}|1+i \sqrt{\omega-1}|^{2}\right)} .
\end{aligned}
$$

Table 1 presents a comparison of our results with experimental measurements and with other theoretical results.

Besides asymmetry parameters, the experiment of Gulley et al [67] measured the total cross sections, $\sigma^{(1)}$ and $\sigma^{(2)}$, and estimated an upper limit for $\sigma^{(3)}$. The $n$-photon detachment cross 

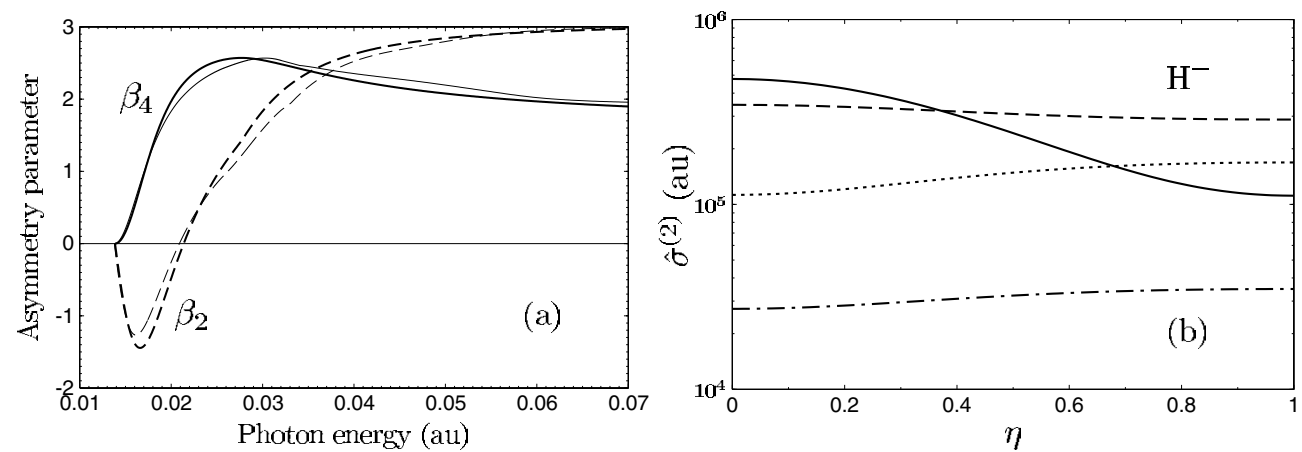

Figure 12. (a) Asymmetry parameters $\beta_{2}$ (thick broken curve) and $\beta_{4}$ (thick full curve) for $\mathrm{H}^{-}$ in the $\delta$-model approach (cf (84)) as functions of photon energy. Thin broken and thin full curves are the results of Sánchez et al (1998) [107]. (b) Ellipticity dependence of the total generalized cross section for two-photon detachment of $\mathrm{H}^{-}$for four photoelectron energies, $E_{p}=\omega-2\left|E_{0}\right|$, chosen to be the same as in figure 4 of [77]. Full curve: $\omega=0.0157$ au ( $=0.566$ in sc. units); long broken curve: $\omega=0.0182 \mathrm{au}(=0.655$ in sc. units); short broken curve: $\omega=0.0284$ au $(=1.025$ in sc. units); chain curve: $\omega=0.043$ au (=1.55 in sc. units).

Table 1. Photoelectron angular distribution asymmetry parameters $\beta_{2}$ and $\beta_{4}$ for two-photon detachment of $\mathrm{H}^{-}$.

\begin{tabular}{llll}
\hline$\omega$ (in sc. units) & $\beta_{2}$ & $\beta_{4}$ & References \\
\hline 0.8620 & $0.7 \pm 0.2$ & $2.0_{-0.2}^{+0.4}$ & Expt. [68] \\
& 0.70 & 2.47 & This work (cf (84)) \\
& 0.65 & 2.31 & Theory [107] \\
1.0027 & $1.3 \pm 0.2$ & $2.4_{-0.2}^{+0.17}$ & Expt. [68] \\
& 1.44 & 2.57 & This work (cf (84)) \\
& 1.10 & 2.47 & Theory [125] \\
& 1.22 & 2.52 & Theory [107] \\
1.5468 & $2.54_{-0.6}^{+0.44}$ & $2.29_{-0.31}^{+0.07}$ & Expt. [67] \\
& 2.71 & 2.20 & This work (cf (84)) \\
\hline
\end{tabular}

Table 2. Generalized cross sections for one-, two- and three-photon detachment of $\mathrm{H}^{-}$for $\omega=1.5468$.

\begin{tabular}{llcl}
\hline$\sigma^{(1), \mathrm{H}^{-}}$ & $\hat{\sigma}^{(2), \mathrm{H}^{-}}$ & $\hat{\sigma}^{(3), \mathrm{H}^{-}}$ & \\
$\left(10^{-17} \mathrm{~cm}^{2}\right)$ & $\left(10^{-48} \mathrm{~cm}^{4} \mathrm{~s}\right)$ & $\left(10^{-79} \mathrm{~cm}^{6} \mathrm{~s}^{2}\right)$ & References \\
\hline $3.6 \pm 1.7$ & $1.3 \pm 0.5$ & $\ll 4.4$ & Expt. [67] \\
3.65 & 0.96 & 0.13 & This work (cf (85)) \\
3.58 & & & Theory [151] \\
\hline
\end{tabular}

section, $\sigma^{(n)}$, is connected with the partial decay rate, $\Gamma^{(n)}$, in the standard way, $\sigma^{(n)}=\Gamma^{(n)} / \mathcal{J}$, where $\mathcal{J}=F^{2} /(8 \pi \omega \alpha)$ is the photon flux (in scaled units) and $\alpha$ is the fine-structure constant. For $n>1$ the 'generalized cross sections', $\hat{\sigma}^{(n)}$,

$$
\hat{\sigma}^{(n)}=\left[\frac{8 \pi \omega \alpha}{F^{2}}\right]^{n-1} \sigma^{(n)},
$$

are convenient for analysing $n$-photon detachment. (In absolute units, the dimension of $\hat{\sigma}^{(n)}$ is $\mathrm{cm}^{2}\left(\mathrm{~cm}^{2} \mathrm{~s}\right)^{(n-1)}$.) In table 2 we present theoretically calculated and experimentally measured [67] cross sections of $\mathrm{H}^{-}$photodetachment for $n=1,2,3$ at $\omega=1.5468$. Tables 1 

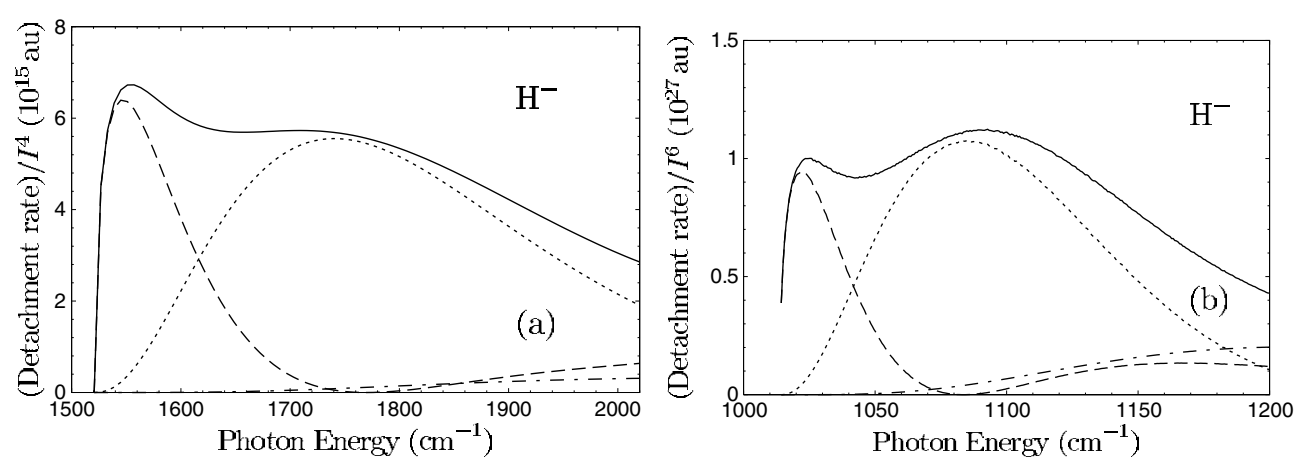

Figure 13. Partial and total detachment rates for $\mathrm{H}^{-}$in a linearly polarized field for $n=4$ (a) and $n=6$ (b). Full curve: total rate; long broken curve: partial rate to the $S$-channel; short broken curve: $D$-channel partial rate; chain curve: $G$-channel partial rate. The contribution of the $I$-continuum channel (for $L=6$ ) is negligible for the frequencies considered and is therefore not shown.
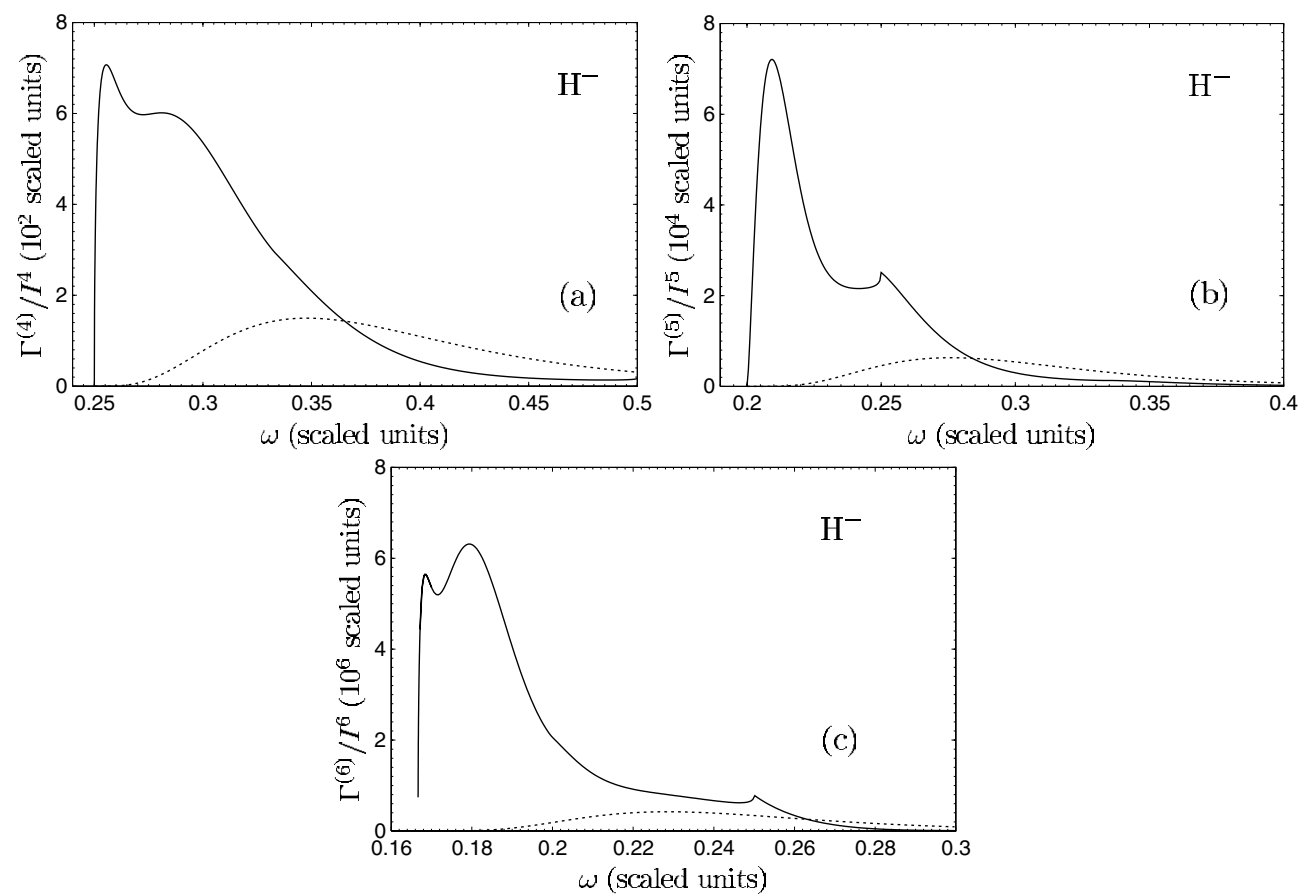

Figure 14. Frequency dependence of the total detachment rates for $\mathrm{H}^{-}$(divided by $I^{n}$; in scaled units) in linearly polarized (full curve) and circularly polarized (short broken curve) fields for (a) $n=4$, (b) $n=5$ and (c) $n=6$. Results for $l=0$ correspond to $G-, H$ - and $I$-continuum channels, respectively.

and 2 demonstrate good agreement of our simple analytical results with both experimental measurements and more sophisticated calculations.

For a more detailed analysis of the accuracy of the $\delta$-model potential in multiphoton calculations for $\mathrm{H}^{-}$, in figure 12 we present the asymmetry parameters $\beta_{2}$ and $\beta_{4}$ (where $\beta_{2}$ is phase-sensitive), and also the ellipticity dependence of the total detachment rate for $n=2$. Our 
results for $\beta_{2,4}$ are in good agreement with the results in figure 2 of Sánchez et al (1998) [107]; the maximum difference between our results and theirs does not exceed $10 \%$. There is similarly good agreement of our results and theirs for the total two-photon rate for $l=1$. Finally, one observes similar agreement between our results for the ellipticity dependence of the total twophoton detachment rate for $\mathrm{H}^{-}$shown in figure 12(b) and those in figure 4 of Nikolopoulos and Lambropoulos [77].

In figure 13(a) we present the total rate for four-photon detachment of $\mathrm{H}^{-}$for $l=1$, calculated using equation (63). Comparison with the $B$-spline multielectron calculations of van der Hart [105] demonstrates the same good agreement and level of accuracy of our results as for the two-photon asymmetry parameters $\beta_{2}$ and $\beta_{4}$ discussed above. Our results even confirm a small dip just above the first minimum (that arises from the interference of $\mathrm{S}$ - and D-wave final state channels), which is slightly more pronounced in figure 13(a) than in figure 5 of [105]. This result disproves an assertion that '... the difference between model potential approaches and descriptions with electron correlations included will be more pronounced in the four-photon detachment spectrum than for lower order detachment' [105]. Moreover, a similarly good agreement (with only about $10 \%$ differences in the positions and heights of the maxima corresponding to the S- and D-wave continuum channels) may be observed by comparing the results for $n=6$ in our figure 13(b) with those in figure 7 of [105].

The total rates $\Gamma^{(n)}$ for $n=4,5$ and 6 are presented in figure 14 over an extended frequency interval (compared with that in [105]) for two polarization states of the laser, $l=0$ and 1 . These results demonstrate the interesting fact that, for high enough above-threshold frequencies (corresponding to the absorption of a few excess photons), the efficiency of detachment by a circularly polarized field becomes higher than that for the case of linear polarization (though both efficiencies are very small). It means that the continuum channel with maximum orbital momentum, $L=n$, is the dominant one for these frequencies. Meanwhile, for smaller $\omega$, the population of this channel is negligible and it is not even visible in figure 14 for $n=6$. This statement may be regarded as a general one for the PT region; we have found it to hold for $n>6$ as well. Finally, our results in figures 13 and 14 are in good agreement (with an accuracy of about $2 \%$ ) with results [102] of one-electron PT calculations of $\Gamma^{(n)}$ for $\mathrm{H}^{-}$(for $2 \leqslant n \leqslant 8$ and 'below-threshold' frequencies, $1 / n \leqslant \omega \leqslant 1 /(n-1))$.

In summary, the above comparisons demonstrate a reasonable agreement of our analytical results with more detailed (and time-consuming) calculations. (See, however, the discussion of figure 5 above.) We are inclined to consider such agreement for multiphoton calculations as excellent, taking into consideration that an accuracy of $10 \%$ or better is beyond the capabilities of recent multiphoton experiments.

\section{Strong field analysis of angular distributions and ED effects}

\subsection{Symmetry properties of angular distributions and elliptic dichroism}

In a strong field, the symmetry properties of ADs (including the ED effect) are essentially the same as in the PT analysis of the previous section since they follow from rather general arguments $[11,49]$. In the dipole approximation, $\boldsymbol{e}$ and $\boldsymbol{n}$ are the only vector parameters of the problem for the case of an unpolarized target atom. Thus, in any PT order (and hence in the nonperturbative regime as well) the $n$-photon amplitude may involve only even (for even $n$ ) or odd (for odd $n$ ) powers of scalar products $\boldsymbol{e} \cdot \boldsymbol{n}$ and $\boldsymbol{e}^{*} \cdot \boldsymbol{n}$. Hence, the AD is invariant to $\boldsymbol{n} \rightarrow \boldsymbol{- n}$. This inversion symmetry stems from parity conservation in the problem considered. Also, the AD is invariant to a rotation by an angle $\pi$ around the direction of $\hat{k}$ (which corresponds to the invariance of results with respect to inversion of the directions 
of the polarization unit vectors $\hat{\epsilon}$ and $[\hat{k} \times \hat{\epsilon}]$, i.e. to the substitutions $e \rightarrow-e, e^{*} \rightarrow-e^{*}$ in the cross section). This symmetry is equivalent to a rotation by $\pi$ around $\hat{\epsilon}$ followed by a rotation by $\pi$ around $[\hat{k} \times \hat{\epsilon}]$. Besides the two mentioned above, no other symmetries exist for ADs produced by an elliptically polarized laser. Moreover, there is a significant asymmetry resulting from the change $\xi \rightarrow-\xi$ that originates from odd powers of the ED factor (cf (71) or (75) and the discussion following equations (69) and (70)). For the $\delta$-model potential, all these general observations follow straightforwardly from the exact results (36) and (38), where the coefficients $\phi_{ \pm p}$ depend only on $l$ and the Bessel functions depend only on the modulus $|e \cdot n|$.

As mentioned above, the $\mathrm{AD}$ is invariant to a simultaneous rotation by an angle $\pi$ around the directions of $\hat{\epsilon}$ and $[\hat{k} \times \hat{\epsilon}]$. Neglecting the ED terms, ADs exhibit an additional symmetry with respect to separate rotations by an angle $\pi$ around either $\hat{\epsilon}$ or $[\hat{k} \times \hat{\epsilon}]$. The ED terms, however, destroy this symmetry and cause the well-known asymmetry of the ADs. Thus, this asymmetry illustrates an interesting physical phenomenon specific to an elliptically polarized field: the light-helicity dependence of multiphoton ADs. Note that if one neglects the coefficients $f_{n}$ with $n \neq 0$ (as is done to reduce the exact results for a $\delta$-model potential to those for the KA), the ED effect (i.e. the asymmetry with respect to $\xi$ ) of the AD vanishes. Finally, we note the general symmetry of ADs with respect to the substitution $\xi, \hat{k} \rightarrow-\xi,-\hat{k}$ (which is equivalent to the change $\xi \rightarrow-\xi$ and a rotation by $\pi$ around either $\hat{\epsilon}$ or $[\hat{k} \times \hat{\epsilon}]$ ), i.e. the equivalence of ADs in two experiments with opposite helicities and directions of the laser beam ( $\mathrm{cf}(71))$.

\subsection{Total and partial rates of $n$-photon detachment}

We analyse here the dependence of the $n$-photon detachment rates, $\Gamma^{(n)}$, on intensity and frequency in the nonperturbative domain of these parameters. For a given $n$, this nonperturbative domain is bordered by the PT regime (for low intensities and/or high frequencies) and the region of the intensity-induced closing of the $n$-photon detachment threshold. We give here examples of the behaviour of the rates between these two extreme cases. Numerical results are obtained using (36) and (38) as well as the angular integral over the differential rate (38), in which $\epsilon$ and $\phi_{n}$ are numerical solutions of equations (27) and (28). Expansion of the amplitude (36) in partial waves allows us to calculate numerically the fixed angular momentum $L$ components of $\Gamma^{(n)}(\boldsymbol{n})$ (i.e. partial $n$-photon rates). The amplitude $\mathcal{A}_{n}$ in (36) involves an infinite summation over $p$, allowing in general any value of $L$ permitted by dipole selection rules. This reflects the possibility that virtual absorption and emission of photons may lead to a final-state angular momentum value that is larger than $n$. This possibility is specific to the nonperturbative regime since the LOPT analysis predicts only a maximum of $n$ quanta of angular momenta absorbed by the ejected electrons. However, our numerical calculations show that for all the values of the parameters in the nonperturbative regime considered by us, the contribution of the continuum channels with $L$ higher than $n$ is extremely small.

In figure 15 we show the two-photon detachment dependence on frequency for the case of linear and elliptic polarization and two (perturbative and strong) laser intensities. We prefer to plot the dependence on the inverse frequency $1 / \omega$ (i.e. wavelength in our scaled units) since it allows a better visualization of the low frequency region, $\omega<1$. As the intensity increases, the ponderomotive shift $U_{p}$ moves the position of the threshold towards larger frequencies. The curves representing the $\mathrm{S}$ and $\mathrm{D}$ rates allow us to understand the modification of the summed two-photon rate. Qualitatively, the $\mathrm{S}$ and $\mathrm{D}$ rates have the same behaviour at all intensities: a fixed number of maxima and minima covering the region from the threshold to the high 

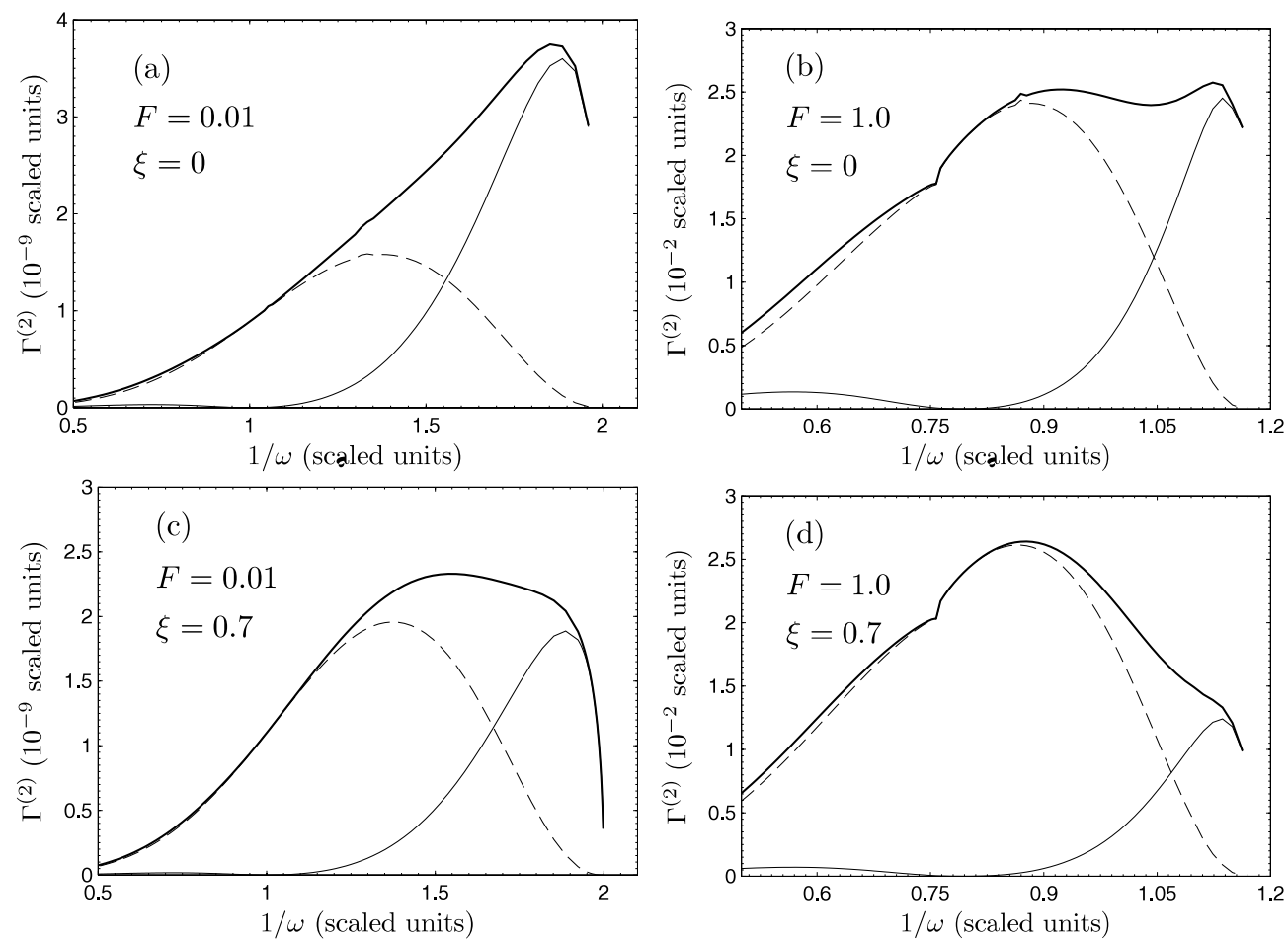

Figure 15. Two-photon detachment rate for the case of linearly polarized $(\xi=0$; $(\mathrm{a}, \mathrm{b}))$ and elliptically polarized $(\xi=0.7$; (c, d)) light as a function of $1 / \omega$ for two intensities: (a, c) $F=0.01$, (b, d) $F=1.0$. Thin full curve: S-wave continuum channel; thin broken curve: D-wave continuum channel; thick full curve: total two-photon rate (sum of S and D channels).

frequency limit. These maxima and minima are related to the oscillatory nature of the Bessel functions appearing in (36). In general, for any fixed $n$, the number of maxima of the partial waves when going from the high frequency, low intensity limit to the threshold ranges from only one maximum for $L=n$ up to $[n / 2]+1$ for the lowest value of $L$ allowed by the dipole selection rules $(0$ for even $n$ and 1 for odd $n$ ). This can be observed in figure 15 where the D-wave channel rate has only one maximum and the $\mathrm{S}$-wave channel rate has two maxima and one minimum. Changing the intensity results in the same number of maxima and minima but changes their frequency location and the magnitude of the rates at the maxima. As the intensity increases, the magnitudes of the maxima of both the total and partial rates increase. Also, the maximum of the $\mathrm{D}$-wave partial rate increases relative to that of the $\mathrm{S}$-wave partial rate so that the total rate changes its shape (versus $1 / \omega$ ) from having a single maximum at low intensity to having a double maximum at high intensities. All these features reflect a complicated, intensity-dependent interference of the different detachment pathways leading to a particular final state of the escaping electron, such as between different L-wave channels contributing to a particular final momentum state, or between LOPT and higher-order processes contributing to a particular electron channel. Since for circular polarization only electrons with $L=n$ can be ejected, as we vary the ellipticity from linear to circular the relative importance of the $L=n$ partial rate must increase. This can be observed by comparing figures 15 (a), (b) for $\xi=0$ to figures $15(\mathrm{c})$, (d) for $\xi=0.7$. For circular polarization the structure of the $n$ photon rate is very simple: $L=n$ is the only allowed angular momentum and the corresponding rate 

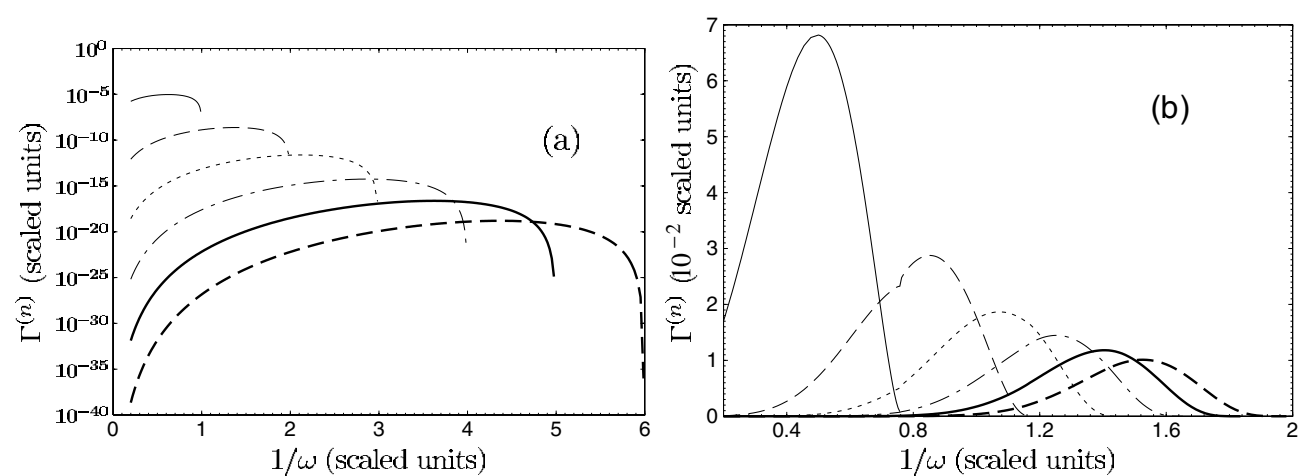

Figure 16. $n$-photon rates for $1 \leqslant n \leqslant 6$ as functions of $1 / \omega$ for circular polarization $(\xi=1)$ at two intensities: (a) $F=0.01$, (b) $F=1.0$. Each $n$-photon detachment rate has only one angular momentum component: $L=n$. Thin full curve: $n=1$; thin broken curve: $n=2$; thin short broken curve: $n=3$; thin chain curve: $n=4$; thick full curve: $n=5$; thick broken curve: $n=6$.
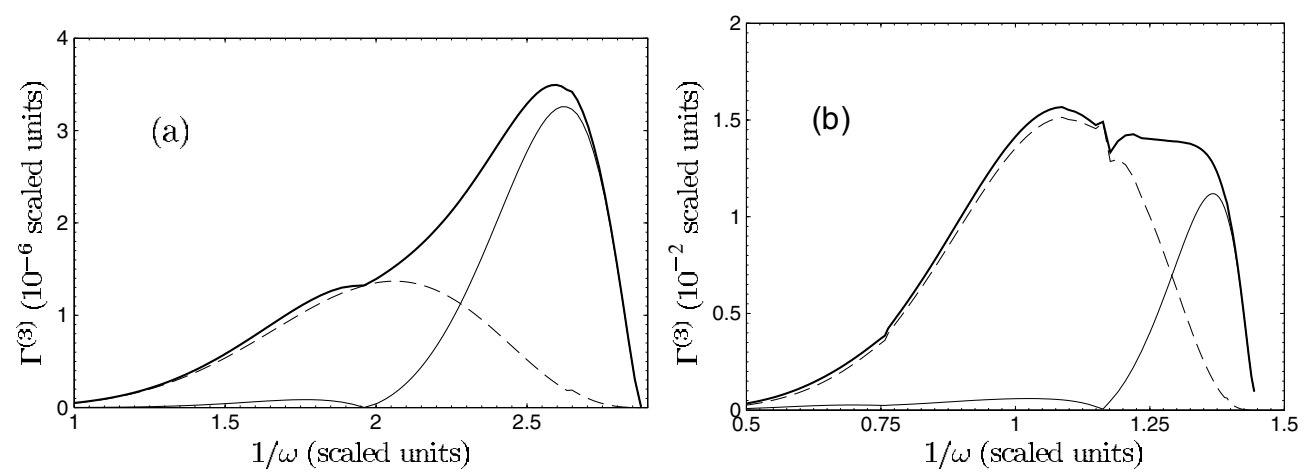

Figure 17. Three-photon detachment rate as a function of $1 / \omega$ for elliptic polarization $(\xi=0.7)$ and two intensities: (a) $F=0.1$, (b) $F=1.0$. Thin full curve: P component; thin broken curve: $F$ component; thick full curve: total three-photon rate (sum of $\mathrm{P}$ and $F$ components).

has only one maximum. Figure 16 shows examples of the dependence of $n$-photon rates on $1 / \omega$ for the case of circular polarization and for the same intensities as in figure 15 .

In figures 17 and 18 we show additional examples of partial wave decompositions for the three- and five-photon rates for the case of elliptical polarization. The three-photon rate has a behaviour that is similar to that of the three-photon rate, with the same number of oscillations in the $\mathrm{P}$ component rate as in the $\mathrm{S}$ component rate for two photons and with the $F$ component rate similar to that of the two-photon $\mathrm{D}$ component rate. A new feature in figure 17 is the sharp variation of the total and partial rates associated with the closing of the two-photon channel. This feature appears at the position $\omega=0.5(1 / \omega=2)$ for low intensity and at higher frequency for larger intensity. One can notice that the size of the feature grows with intensity. The same features can be seen in figure 18 at the closing of the two-, three- and four-photon detachment channels. Common to figures 15,17 and 18 is the fact that, at high frequency, the high $L$ component is dominant, while closer to the threshold, other $L$ partial waves may be dominant. In the region very close to the threshold, the onset of the Wigner threshold law regime is realized in which the lowest $L$ partial rate dominates over higher $L$ partial rates, which fall off faster towards zero, according to Wigner's law. 

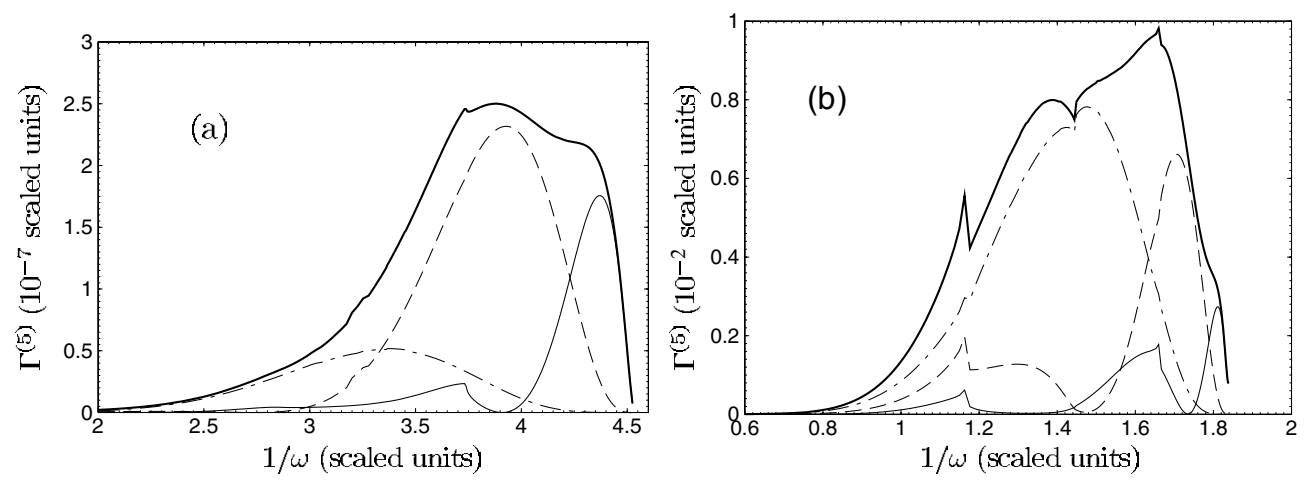

Figure 18. Same as figure 17 but for five-photon detachment, where now the chain curve denotes the $\mathrm{H}$-wave partial rate and the full curve is the sum of the P-, F- and H-wave partial rates.

The intensity dependence of the $n$-photon rates as well as that of the partial rates looks very similar to their dependence on the inverse frequency. The reason is that, as may be seen in (B.1), the matrix elements in the system of equations (27) that determine the coefficients $\phi_{n}$ depend on $F$ and $1 / \omega$ in a similar way. There is an additional dependence on $\omega$ in the coefficients of the series but, for the parameters considered, this dependence is very weak. We have the situation of a quasiscaling with the parameter $F / \omega^{2}$; therefore the dependence on $F$ is similar to that on $1 / \omega^{2}$. In figure 19 we present the dependence of $\Gamma^{(n)}$ (and of its partial rates for particular values of $L$ ) on the laser amplitude $F$ for $n=2,3$ and 5, respectively, for the same ellipticity $(\xi=0.7)$ and for two frequencies: $\omega=0.8$ and 1.5 . The intensity is varied from small, perturbative values up to the value for which the $U_{p}$ shift closes the particular $n$-photon channel. Near the two- and three-photon thresholds, we see the much faster increase of the low $L$ rates, $\mathrm{S}$ and $\mathrm{P}$, followed by a region where the $\mathrm{D}$ and $F$ rates dominate. In the low intensity part (not visible in the figures), which of the partial rates is dominant depends on the value of $\omega$; this information can be extracted from the previous figures showing the $\omega$ dependence for low intensities. From figures 19(e), (f) one can see that, for higher numbers of photons, the lower $L$ components have increasingly more oscillations while the component with $L=n$ has the least rapid variation and, except for threshold-induced anomalies, only one broad maximum. One can notice these distinct TAs in both the partial rates and the total rate. They are associated with the closing of the lower-order detachment channels [135].

\subsection{Three-dimensional angular distributions}

As in the PT analysis, we find it more instructive to show 3D plots of the differential rates $\Gamma^{(n)}(\boldsymbol{n})$. These rates are obtained using the exact result given by equations (37) and (38). Since we are interested in the change of the AD as the intensity is varied over a large range, thus leading to large variations of the rates, we rescale our results such that all of the 3D figures have approximately the same size. Figure 20 shows the ADs for two- and three-photon detachment as the intensity is increased from low intensity up to that which closes, respectively, the two- and three-photon detachment channels. For low perturbative intensities, the electrons are ejected mainly along the major polarization axis. However, as the intensity increases, the interplay between the various $L$ components can lead to significantly different ADs. In the simplest cases of two- and three-photon detachment, the interplay between the S and D (or P and $F$ ) components in the region preceding the onset of the Wigner threshold law regime can 

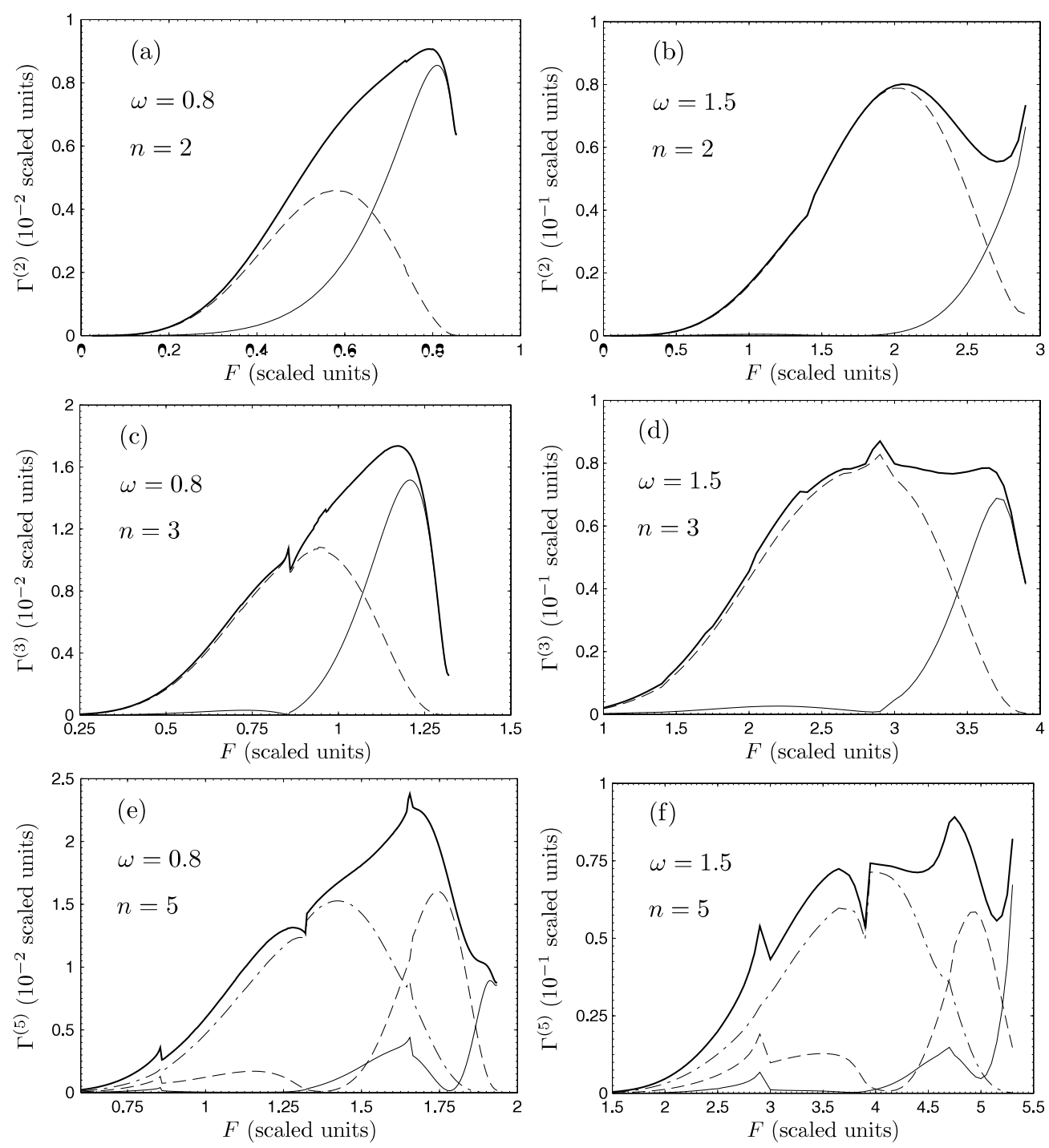

Figure 19. $n$-photon detachment rate as a function of laser amplitude, $F$, for the case of elliptic polarization ( $\xi=0.7$ ) for two frequencies, $\omega=0.8$ (left panels) and $\omega=1.5$ (right panels). Thick full curves represent total $n$-photon rates. (a, b) Thin full curve-S-wave component; thin broken curve-D-wave component. (c, d) Thin full curve-P component; thin broken curve- $F$ component. (e, f) Thin full curve- $\mathrm{P}$ component; thin broken curve $-F$ component; thin chain curve- $H$ component.

lead to a cancellation of the detachment amplitude along the major polarization axis for certain values of the intensity and the frequency [78]. This effect is illustrated in figure 20 where one sees that, in the region of intensities shown in the third and fourth rows, the probability for two- and three-photon detachment along the $\hat{\epsilon}$ axis passes though zero. This situation can be realized for any polarization that allows the existence of several angular momenta in the final state, i.e. for any polarization except circular. For circular polarization, the AD is always that corresponding to an $L=n$ partial wave and does not change its shape as either the frequency 

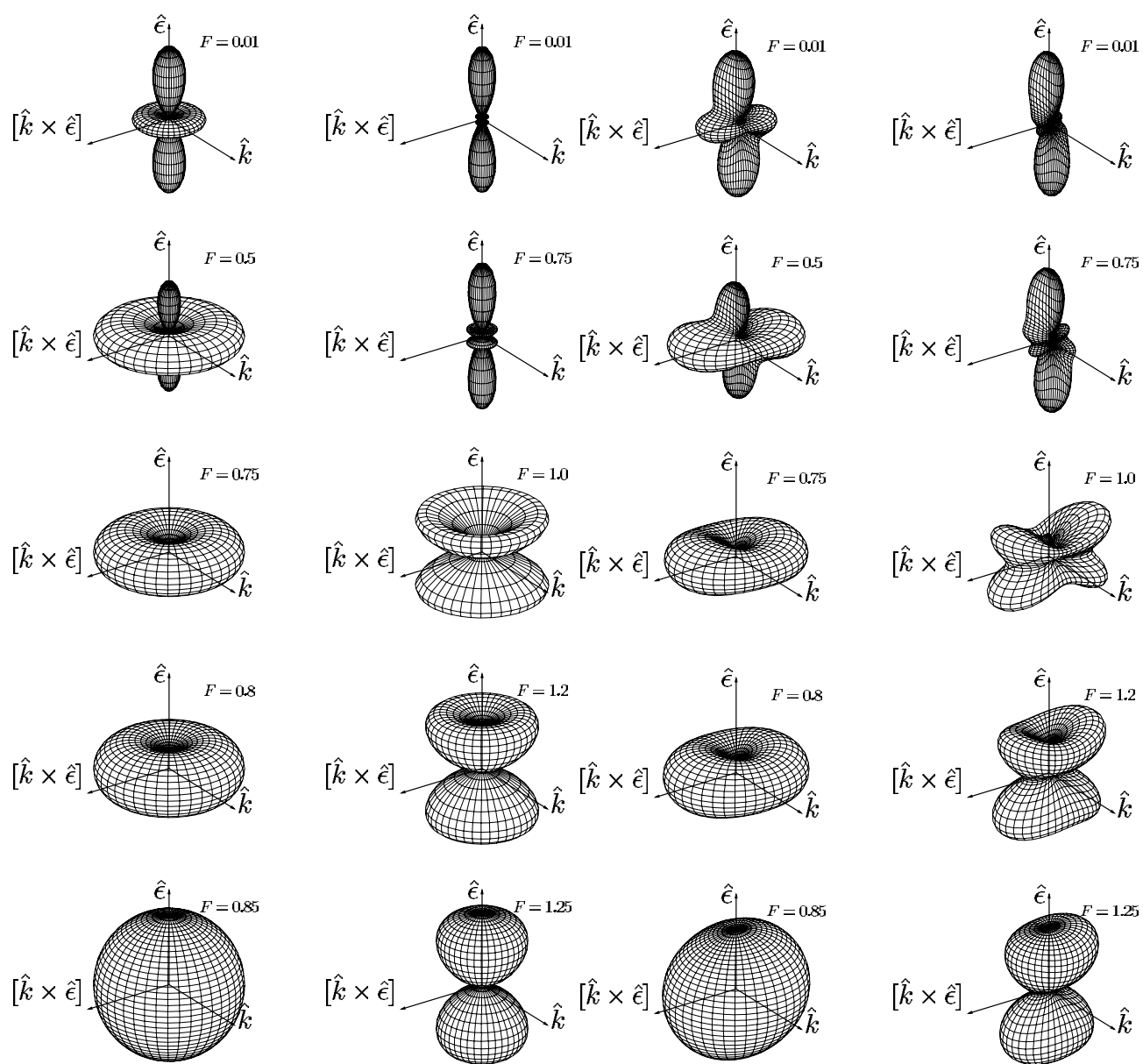

Figure 20. Two- and three-photon detachment ADs (i.e. $n=2,3$ ) as functions of the laser amplitude $F$ for the fixed frequency, $\omega=0.8$, and two different polarizations. First column: $n=2, \xi=0.0$ (linear polarization); second column: $n=3, \xi=0.0$; third column: $n=2$, $\xi=0.7$; fourth column: $n=3, \xi=0.7$. For each case, five values of $F$ are given, in which the lowest, $F=0.01$, corresponds to the perturbative regime and the highest is just at the intensityinduced closing of the $n$-photon channel. The different figures have been scaled in order to have the same size.

or the intensity is varied. The unusual ADs obtained for linear and elliptic polarization can be understood as resulting from quantum interference between the different angular momentum states in the intermediate region between the perturbative regime and the Wigner threshold law regime. For the highest value of the intensity shown in each figure, which in each case is close to the corresponding threshold, we see that the $\mathrm{S}$ and $\mathrm{P}$ waves are dominant for two- and three-photon processes, respectively, in agreement with Wigner's threshold law (cf the bottom row of figure 20).

The oscillation pattern of the ADs for the case of linear polarization was mentioned in earlier quasiclassical (saddle point) calculations (see equation (53) in [1](c) and the discussion after equation (23) in [1](b)) and was analysed later in more detail by Gribakin and Kuchiev [128] using the Keldysh approach. In these analyses the angular momentum components of the escaping electron were not resolved; instead, the oscillation pattern is 
Table 3. Photoelectron AD asymmetry parameters $\beta_{2 k}$ for two-photon detachment of $\mathrm{H}^{-}$by the linearly polarized field of frequency $\omega=0.758(\lambda=2.15 \mu \mathrm{m}) \cdot 10^{n} \equiv(n)$.

\begin{tabular}{llllll}
\hline$I\left(\mathrm{~W} \mathrm{~cm}^{-2}\right)$ & $\beta_{2}$ & $\beta_{4}$ & $\beta_{6}$ & $\beta_{8}$ & References \\
\hline $1.3(11)$ & $-5.49(-1)$ & 1.97 & $-4.55(-2)$ & $4.37(-4)$ & Equations (38), (37) \\
& $-3.62(-1)$ & 1.84 & $-4.18(-2)$ & $3.95(-4)$ & {$[153]$} \\
& $2.35(-1)$ & 2.41 & $0.34(-2)$ & $58.9(-4)$ & {$[128]^{\text {a }}$} \\
& $2.22(-1)$ & 2.39 & $-5.59(-2)$ & $5.41(-4)$ & Equations (38), (45) \\
$6.5(11)$ & -1.32 & $3.63(-1)$ & $-1.20(-2)$ & $1.75(-4)$ & Equations (38), (37) \\
& -1.19 & $2.87(-1)$ & $-0.77(-2)$ & $0.91(-4)$ & {$[153]$} \\
& -1.37 & $3.92(-1)$ & $-1.08(-2)$ & $2.67(-4)$ & {$[128]^{\text {a }}$} \\
& -1.42 & $4.34(-1)$ & $-1.58(-2)$ & $2.52(-4)$ & Equations (38), (45) \\
\hline
\end{tabular}

${ }^{\text {a }}$ Entries for [128] are taken from table II of [153].

interpreted as stemming from the interference between contributions of two saddle points corresponding to two peak values of the field amplitude over the laser period. Recently the suppression of electron yield along the direction of linear laser polarization has been measured by Reichle et al [71] in two-photon detachment of $\mathrm{H}^{-}$at $\omega=0.758$, and their experimental results (for two laser intensities, $1.3 \times 10^{11}$ and $6.5 \times 10^{11} \mathrm{~W} \mathrm{~cm}^{-2}$ ) were found to be in agreement with the KA results [128]. Very recently, more sophisticated calculations have been performed [153] (based on the numerically intensive methods described in [126]) with results that differ only slightly from those in [128], primarily for the lower intensity. In table 3 we compare the above-mentioned results for the AD asymmetry parameters with our results obtained using the exact equations (38) and (37) and also our results using the 'exact' KA amplitude (45) (i.e. beyond the adiabatic approach used in [128]). For the lower intensity, one sees that our exact results are in reasonable agreement with those in [153], while for the higher intensity all results are in reasonable agreement.

Figure 21 shows the $\mathrm{AD}$ of all the ejected electrons, $\Gamma(\boldsymbol{n})=\sum_{n} \Gamma^{(n)}(\boldsymbol{n})$, regardless of their energy. For low intensities, the two-photon channel is the dominant process and the total $\operatorname{AD} \Gamma(\boldsymbol{n})$ is very similar to that for two-photon detachment. For higher intensities, the ATD rates become increasingly more important. Several partial rates contribute equally to $\Gamma(\boldsymbol{n})$. As the intensity increases, $\Gamma(\boldsymbol{n})$ has a very different form from those of the individual $n$-photon rates $\Gamma^{(n)}(\boldsymbol{n})$ shown in figure 20. We see in particular that in the region of intensities for which the two- or three-photon rates have close to zero electrons ejected along the major polarization axis, $\Gamma(\boldsymbol{n})$ does not preserve this property. Higher-order processes are more important and completely mask the effect. Indeed, as the intensity increases, $\Gamma(\boldsymbol{n})$ is increasingly localized along the major polarization axis $\hat{\epsilon}$.

\subsection{Elliptic dichroism in a strong field}

To analyse the frequency and intensity dependence of ED in the nonperturbative regime, we use the same ED parameter, $\delta^{(n)}$, as in the LOPT case, (77), where the differential rates are measured in the direction of the geometrical maximum of the effect (cf figure (1) and equation (71)): $\theta=\pi / 2$ (i.e. in the polarization plane) and $\alpha=\beta=\pi / 4$ (i.e. halfway between the major and minor axes of the polarization ellipse). We also define the total ED parameter for the total AD $\Gamma(\boldsymbol{n}), \delta^{(t o t a l)}$, by replacing the $n$-photon $\mathrm{AD} \Gamma^{(n)}(\xi ; \boldsymbol{n})$ in $(77)$ by $\Gamma(\xi ; \boldsymbol{n})=\sum_{n} \Gamma^{(n)}(\xi ; \boldsymbol{n})$.

4.4.1. Frequency dependence. In figure 22 we present the frequency dependence of $\delta^{(n)}$ with $n=2,3,4,5$ for three nonperturbative intensities. Similar results for the PT regime were discussed in section 3.2 (figure 10). Aside from the frequency shift of the entire structure of 

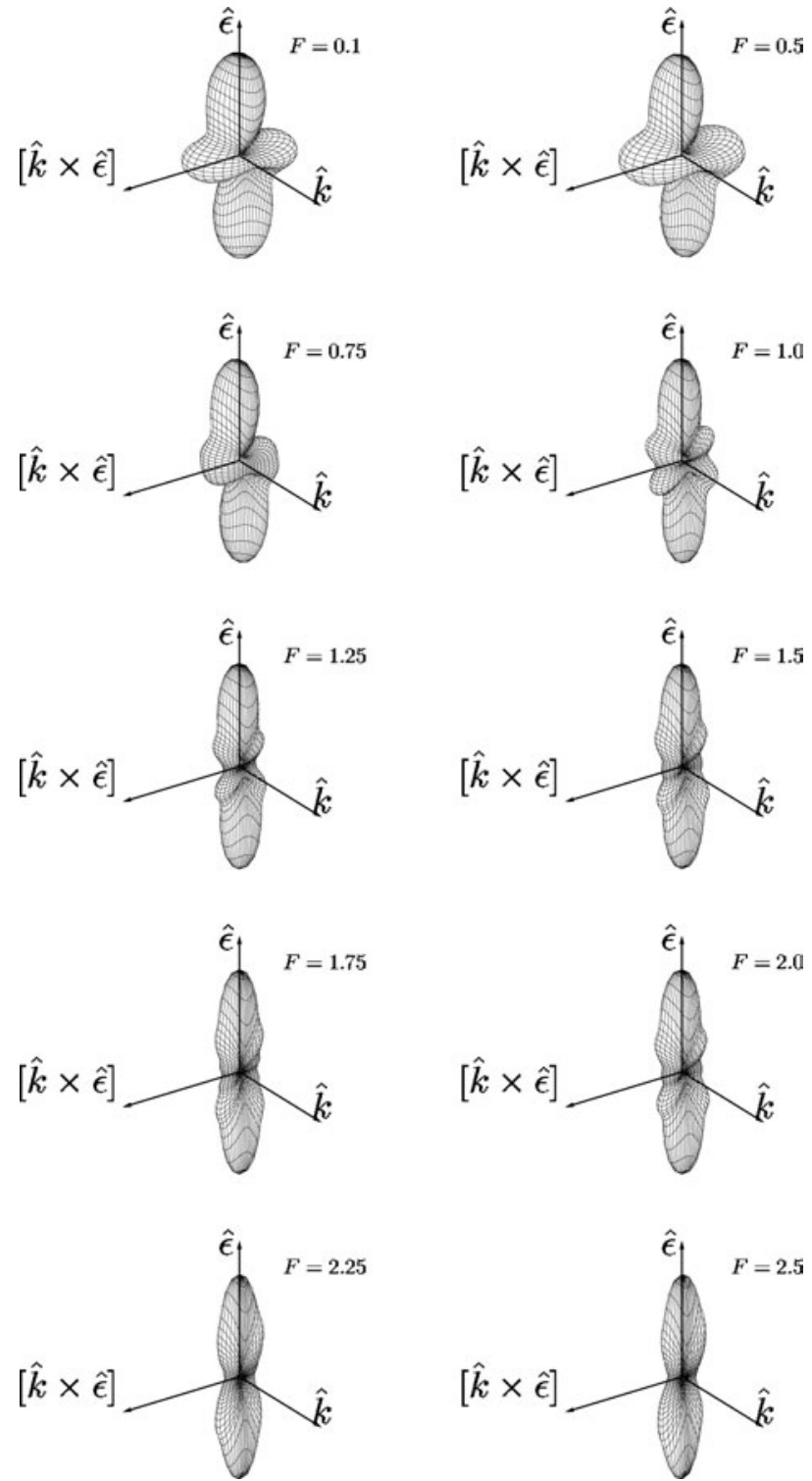

Figure 21. Total detachment ADs (summed over $n$ ) at $\omega=0.8$ and $\xi=0.7$ for ten values of the laser amplitude, $F$, as indicated in the figure. The different figures have been scaled in order to have the same size.

the curves due to the $U_{p}$ shift, one also sees that the ED in the three- and five-photon detachment cases is nonzero even in the region $1 / 3<\omega<1 / 2$ and $1 / 5<\omega<1 / 4$, respectively, which contrasts with the perturbative case. The ED in these frequency regions increases with the laser intensity owing to higher-order processes which, by interference with the lowest-order ones, produce the ED effect. The oscillations of $\delta^{(n)}$ can be traced to the oscillatory nature of the Bessel functions that determine the ADs, as discussed in section 3.2. Note that, in the limit of the Wigner law regime, in which one L component is dominant, the ED tends to zero since the $\mathrm{AD}$ in this region is that of a pure $\mathrm{S}$ or $\mathrm{P}$ distribution and all interference effects disappear. 

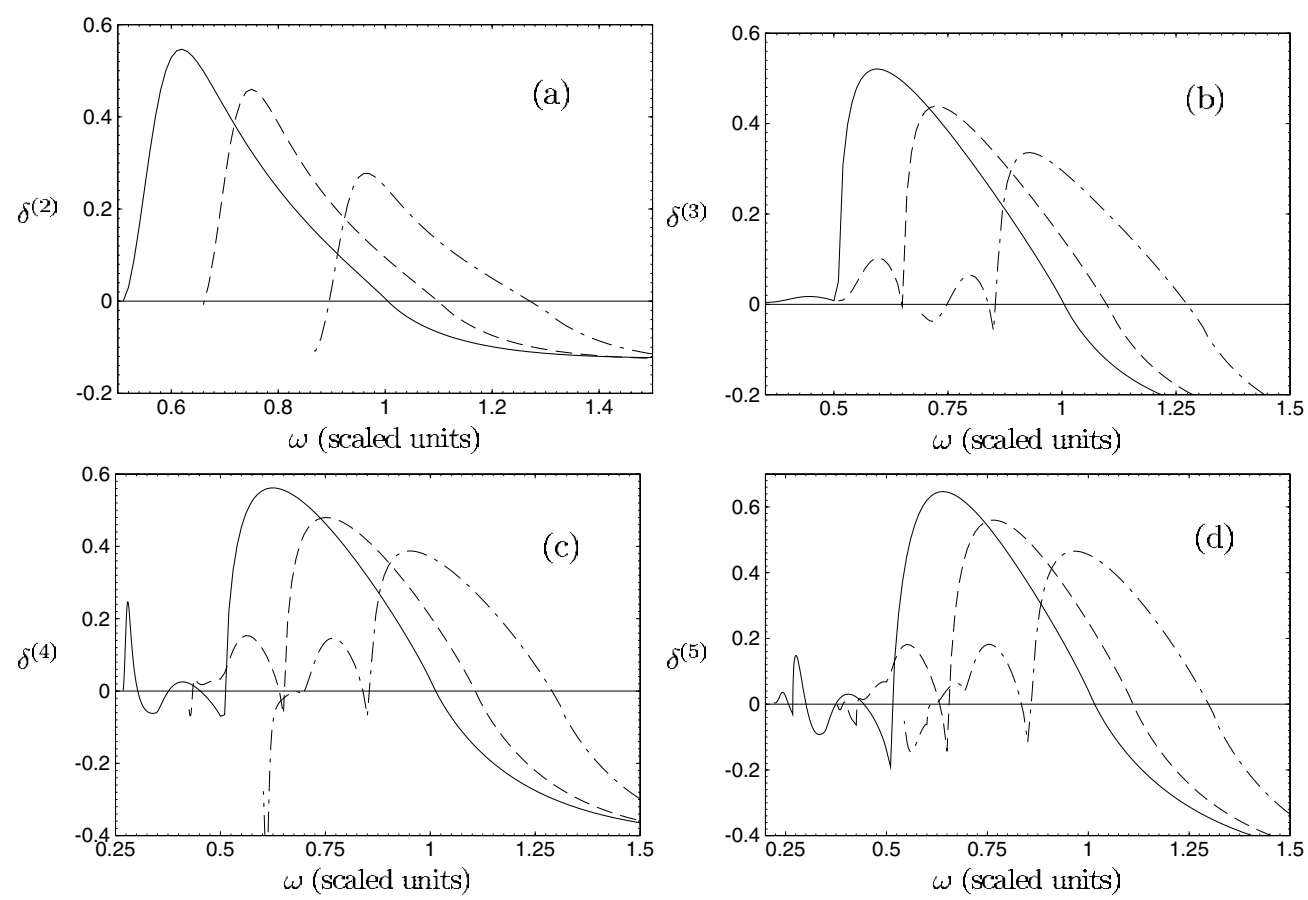

Figure 22. Frequency dependence of the ED parameter $\delta^{(n)}$ for elliptically polarized light $(|\xi|=0.7)$ and for three nonperturbative intensities. Full curve: $F=0.1$; broken curve: $F=0.5$; chain curve: $F=1.0$. (a) $n=2$; (b) $n=3$; (c) $n=4$; (d) $n=5$.

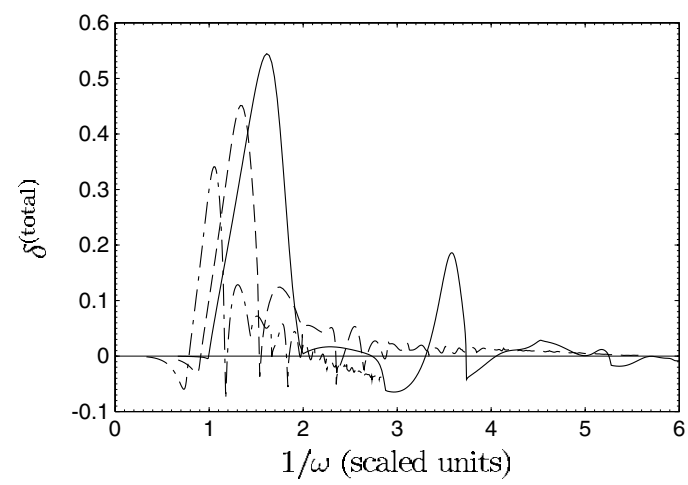

Figure 23. The total ED parameter for $|\xi|=0.7$ plotted versus $1 / \omega$, for three intensities. Full curve: $F=0.1$; broken curve: $F=0.5$; chain curve: $F=1.0$.

Figure 23 shows the total ED parameter as a function of $1 / \omega$ for three intensities. The large maxima can be identified as arising from the large ED effect in a single $n$-photon channel. For low frequency the contributions of a large number of channels, each having a large number of oscillations, tends to reduce the ED effect in $\Gamma(\boldsymbol{n})$. However, for higher intensity $(F=1)$ we note an interesting effect: towards low frequencies the ED parameter shows a decreasing trend which does not stop at zero but continues increasing in absolute value towards negative values. This region corresponds to large maxima (in absolute value) in the $n$-photon ED parameters 


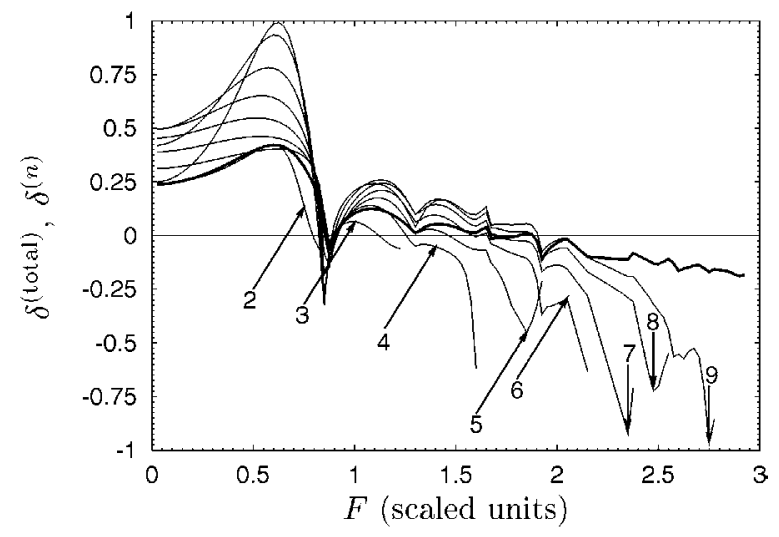

Figure 24. ED parameter as a function of the laser amplitude, $F$, for $\omega=0.8$ and $|\xi|=0.7$. Thick curve: total ED parameter; thin curves: $n$-photon ED parameters with $n$ indicated in the figure.

that appear just before the onset of the Wigner law regime for each $n$-photon process. The magnitudes of these maxima increase with intensity and lead to an overall decreasing trend in the total ED.

4.4.2. Intensity dependence. The ED of different $n$-photon detachment processes, as well as the total ED, are plotted in figure 24 as functions of $F$ for $\omega=0.8$. We can see large variations of the ED parameter for the various $n$-photon rates, which can be considerably larger than those of the total ED parameter. For each $n$-photon process, the dichroism has an oscillatory variation starting in the perturbative region and ending in the threshold regime. A feature common to all the examples presented is the deep minimum present in the $n$-photon ED parameter near the closing of the high $n$ channels. As for the $\omega$ dependence, these minima lead to a decreasing trend in the total ED parameter for high intensities. This can be observed in figure 25(a) where we plot the ED parameter, $\delta$, corresponding to the total detachment yield for several different frequencies. Even though a higher intensity can significantly change the magnitude of the asymmetry, the scale on which these changes appear is strongly related to the magnitude of the asymmetry in the perturbative domain of intensities. For example, at $\omega=0.4$, the asymmetry is exactly 0 at low intensity and does not exceed 0.1 at high intensities well into the nonperturbative regime. In contrast, for $\omega=0.8 \operatorname{each} \delta^{(n)}(n \geqslant 2)$ has a considerable magnitude at low intensities and consequently so does the total $\delta$, including also as one increases the intensity. Figure 25(a) shows that the ED effect is important in the range of intermediate and high frequencies, while for smaller frequencies $(\omega=0.5$ and 0.4$)$ the ED parameter oscillates with smaller amplitudes. Another feature that can be seen in figure 25(a) is the generally decreasing trend of the asymmetry (extending to negative values) that is common to all frequencies considered. The decrease continues below the value 0 so that the absolute value of the asymmetry increases at higher $F$. This trend is especially interesting at the low frequency, $\omega=0.4$, where it appears that an increase of $F$ will cause a significant asymmetry in spite of the small value of $\delta$ at small and medium values of $F$.

One would expect a decrease of the ED effect as the intensity increases, due to the decrease of the relative importance of the binding potential. It has already been mentioned that it is essential to account accurately for the atomic potential (beyond the KA) in order to model the ED effect. One might therefore assume that, as the intensity increases, the relative importance of the binding potential decreases and thus the ED effect decreases. However this assertion is 

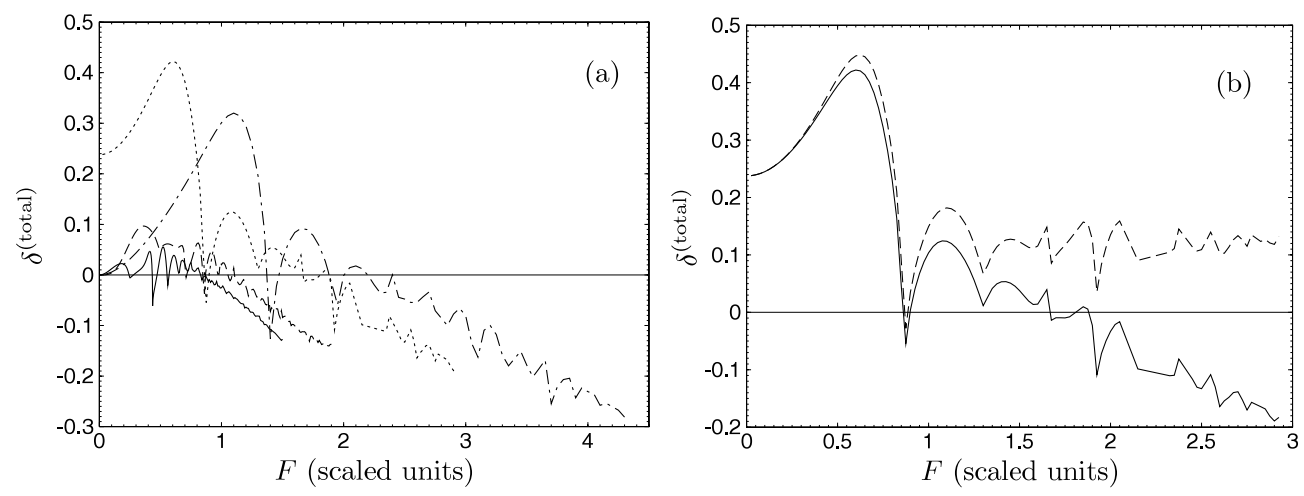

Figure 25. Total ED parameter as a function of the laser amplitude, $F$, for $|\xi|=0.7$. (a) The results for different frequencies (full curve: $\omega=0.4$; broken curve: $\omega=0.5$; chain curve: $\omega=0.8$; dotted curve: $\omega=1.0$ ). (b) The result for $\omega=0.8$ including (full curve) and neglecting (broken curve) the imaginary part of the quasienergy.

contradicted by our results shown in figure 25(a), and therefore a different mechanism must be responsible for the nonzero dichroism observed at high intensities. Aside from the fact that the binding potential leads to nonzero coefficients $\phi_{n}$ having imaginary parts, there is an additional reason for the origin of the ED effect. The imaginary part of the ATD amplitude which multiplies the kinematic factor (71) to produce the ED terms in $\Gamma^{(n)}(\boldsymbol{n})$ can appear due to the complex part of the quasienergy, as measured by $\Gamma=-2 \operatorname{Im} \epsilon$. $\Gamma$ is small at low intensities and cannot affect the ED significantly. However, for high intensities, $\Gamma$ increases very sharply and its contribution to the ED parameter increases correspondingly. We show the importance of this contribution in figure 25(b), where we plot the total ED parameter calculated with the exact quasienergy and with the approximation $\operatorname{Im} \epsilon=0$. (Note that the latter approximate calculation is done using the exact values of the complex coefficients $\phi_{n}$, and therefore the potential is taken into account beyond the KA.) The two results are very close to each other for low intensities, but differ significantly for high intensities, where $\operatorname{Im} \epsilon$ is large. While the exact calculation shows the decreasing trend, the one that neglects $\operatorname{Im} \epsilon$ decreases and then stays approximately constant as $I$ increases. We can conclude that the increasing trend (in absolute value) as intensity increases relates to the direct contribution of $\operatorname{Im} \epsilon$.

\section{Photodetachment in a strong, high frequency field $(\omega>1)$}

In many cases the photon energy of the fundamental or lower harmonics of existing intense lasers exceeds the binding energy of a weakly bound electron in a negative ion. In such cases the 'high frequency' regime of their decay is realized when the direct laser detachment channel is open, i.e. $\omega>1$. Although we have already mentioned in section 4 some peculiarities of the $\omega$ and $F$ dependences of the total rates and ADs for the case of $\omega>1$, in the present section we analyse this important case in more detail. This question is closely related to the widely discussed general problem of strong field atomic stabilization, i.e. to the somewhat counterintuitive regime of atomic ionization in a high frequency field in which the decay probability appears to be a decreasing function of laser intensity. Although theoretical models of such stabilization were suggested more than 10 years ago $[154,155]$ (see also [156, 157] as well as recent reviews [158, 159]), there still does not exist an exact solution of this problem for an electron bound by Coulomb forces in a superstrong laser field. Thus there does not 
exist a complete understanding of the dependence of this interesting nonlinear phenomenon on the atomic and field parameters in the ultrastrong field limit. In particular, the theoretical models predict an unlimited (possibly oscillatory) decrease of the ionization rate $\Gamma$ as the field amplitude increases (see [159]). On the other hand, the results of numerical calculations have demonstrated that the decrease of decay rates for a Coulomb potential [157, 160] covers only a limited interval of laser intensities. Moreover, existing experiments on atomic stabilization [161] provide only limited information on its intensity dependence. Although stabilization was originally identified as a property of decay rates (quasistationary stabilization, QS), in recent years the wavepacket version of stabilization (or 'dynamic stabilization', DS) $[156,162]$ has attracted interest. DS originates from the pulse form of a laser field rather than from any intrinsic property of the atom in a strong monochromatic field. Thus DS means that the total ionization probability at the end of a fixed-duration laser pulse does not approach unity with increasing peak intensity. However, a detailed analysis [163] (see also [160]), which uses the QQES as an adiabatic basis for the laser pulse, has shown that DS has essentially the same (quasistationary) origin as QS.

Though short-range potential models are deemed to be appropriate for the study of QS, analyses employing them sometimes show contradictory results. In particular, for the onedimensional $\delta$ model, there have been claims both for the existence of QS [164] and for its non-existence [165]. The absence of QS for the three-dimensional $\delta$-model potential in a circularly polarized field is claimed in [166]. The more exact analysis in [167] does not give a definitive result for the high intensity limit. Finally, a number of authors deny the existence of stabilization based on formal mathematical arguments, in particular, of QS for ionization from a $\delta$ potential [168] and of DS in pulsed fields [169]. However, these arguments take the form of existence theorems valid for $I \rightarrow \infty$ and do not restrict the existence of a decreasing (stabilization-like) trend of $\Gamma(I)$ in a limited interval of intensities. A recent exact analysis of the QS problem for an electron in a $\delta$-model potential [134] shows that precisely this situation is realized in the nonperturbative regime (up to the closure of the one-photon channel) for above-threshold frequencies $(\omega>1)$ for any polarization state of the laser field. The lack of QS in an asymptotically strong field follows from a more general statement [170] on the equivalence of the effect of a superstrong monochromatic field on a weakly bound electron to that of a strong static electric field (in which case QS is clearly absent).

In what follows, we examine the frequency and intensity dependence of the detachment rates in the high frequency regime. We first examine the perturbative (in intensity) behaviour of the one-photon detachment cross section, showing that a decrease with increasing intensity occurs even in the perturbative region. This decrease (or stabilization) breaks down at the closing of the one-photon threshold. Our exact results show in fact that stabilization-type behaviour occurs in the vicinity of each $n$-photon threshold. We also present exact results for the ADs in the high frequency regime.

\subsection{Higher-order corrections to the one-photon detachment cross section}

For a high frequency field, the PT expansion of the decay rate may be applicable up to high intensity, $I \geqslant 1$. Therefore, we first present analytical results for the intensity-dependent corrections, $\sim I$ and $\sim I^{2}$, to the well-known photodetachment cross section, $\sigma_{0}$, for a shortrange potential:

$$
\frac{\mathrm{d} \sigma_{0}}{\mathrm{~d} \Omega}=\frac{3}{4 \pi} \sigma_{0}|e \cdot n|^{2}, \quad \sigma_{0}=\frac{32 \pi \alpha}{3 \omega^{3}}(\omega-1)^{3 / 2} .
$$

Since the PT expansion for the complex quasienergy $\epsilon$ (and, therefore, for decay rates in a monochromatic laser field) is convergent [143, 171], it means that an exact, strong laser 
field generalization of (86), $\mathrm{d} \sigma^{(1)} / \mathrm{d} \Omega$, has a regular, convergent $\mathrm{RS}$ expansion in the (scaled) intensity, $I=F^{2}$, for not too high $I$ :

$$
\frac{\mathrm{d} \sigma^{(1)}}{\mathrm{d} \Omega}=\frac{\mathrm{d} \sigma_{0}}{\mathrm{~d} \Omega}+I \Delta \sigma_{1}+I^{2} \Delta \sigma_{2}+\cdots
$$

Moreover, in the BW version of an expansion like (87) (see (89) below), the parameters $\Delta \sigma_{i}=\Delta \sigma_{i}(\epsilon)$ are $I$-dependent (owing to their dependence on $\epsilon=\epsilon(I)$ ). Thus, in the same order, $\sim I^{n}$, the accuracy of the BW expansion may be substantially higher than that of the RS expansion since the $n$ th-order BW result includes summations of many infinite subsequences of the RS expansion, which originate from the RS expansions of the parameters $\Delta \sigma_{i}(\epsilon)$ in $I$.

The standard PT calculation of even the first RS correction, $\Delta \sigma^{(1)}$, is not a simple problem since in such an analysis in addition to the calculation of third-order Feynman diagrams (which correspond to the re-emission of a photon) one must also take proper account of the so-called 'secular' and normalization terms, which occur in higher orders of PT (see, e.g., [172]). Moreover, the contributions of separate Feynman diagrams involve (non-regularizable) singularities that cancel only in the total amplitude [173]. In our analysis the problem is simplified because we start from the result (38) for $n=1$, i.e. we deal directly with the exact amplitude $\mathcal{A}_{1}$, thereby avoiding PT calculations of separate Feynman diagrams, etc. To obtain the BW PT expansion of $\mathrm{d} \sigma^{(1)} / \mathrm{d} \Omega$ up to terms $\sim I^{2}$, we expand the Bessel functions in the amplitude $\mathcal{A}_{1}$ in (36) up to terms $\sim F^{5}$. Note that only the coefficients $\phi_{n}$ with $n=0$ and \pm 1 contribute for this case. The final $\mathrm{BW}$ result is

$$
\begin{aligned}
\mathcal{A}_{1}(E)=\mathrm{i} \frac{F}{\omega^{2}} & \sqrt{E-\omega}\left\{\phi_{0}\left[1-\frac{F^{2}}{2 \omega^{4}}(\omega-E)|\boldsymbol{e} \cdot \boldsymbol{n}|^{2}+\frac{F^{4}}{12 \omega^{8}}(\omega-E)^{2}|\boldsymbol{e} \cdot \boldsymbol{n}|^{4}\right](\boldsymbol{e} \cdot \boldsymbol{n})\right. \\
& \left.+\phi_{1}\left[1-\frac{F^{2}}{2 \omega^{4}}(\omega-E)|\boldsymbol{e} \cdot \boldsymbol{n}|^{2}\right]\left(\boldsymbol{e}^{*} \cdot \boldsymbol{n}\right)-\phi_{-1} \frac{F^{2}}{6 \omega^{4}}(\omega-E)(\boldsymbol{e} \cdot \boldsymbol{n})^{3}\right\},
\end{aligned}
$$

where $\phi_{ \pm 1}$ and $\phi_{0}$ are given by (B.5) and (B.6) and their expansions up to $\sim F^{4}$ contribute to $\mathcal{A}_{1}(E)$. The resulting three-term BW expansion for the one-photon detachment cross section may be written as follows:

$$
\begin{aligned}
\frac{\mathrm{d} \sigma_{B W}^{(1)}}{\mathrm{d} \Omega}=16 \pi & \alpha\left|\phi_{0}\right|^{2} \frac{|\omega-E|^{3 / 2}}{\omega^{3}}\left\{|\boldsymbol{e} \cdot \boldsymbol{n}|^{2}-\frac{F^{2}}{\omega^{4}} \operatorname{Re}\left[(\omega-E)|\boldsymbol{e} \cdot \boldsymbol{n}|^{4}+l \frac{\mathcal{D}_{1}(E-\omega)\left(\boldsymbol{e}^{*} \cdot \boldsymbol{n}\right)^{2}}{\sqrt{E-2 \omega}-1}\right]\right. \\
& +\frac{F^{4}}{\omega^{8}}\left[\frac{l^{2}}{4}|e \cdot \boldsymbol{n}|^{2}\left|\frac{\mathcal{D}_{1}(E-\omega)}{\sqrt{E-2 \omega}-1}\right|^{2}+\frac{|\boldsymbol{e} \cdot \boldsymbol{n}|^{6}}{4}\left(|\omega-E|^{2}+\frac{2}{3} \operatorname{Re}(\omega-E)^{2}\right)\right. \\
& +l|\boldsymbol{e} \cdot \boldsymbol{n}|^{2}\left[(\omega-\operatorname{Re} E) \operatorname{Re}\left(\frac{\mathcal{D}_{1}(E-\omega)\left(\boldsymbol{e}^{*} \cdot \boldsymbol{n}\right)^{2}}{\sqrt{E-2 \omega}-1}\right)\right. \\
& \left.+\operatorname{Re}\left(\frac{\mathcal{D}_{1}(E+\omega)}{\sqrt{E+2 \omega}-1} \frac{\omega-E}{6}(\boldsymbol{e} \cdot \boldsymbol{n})^{2}\right)\right] \\
& \left.\left.-l \operatorname{Re}\left(\frac{\mathcal{D}_{2}(E-\omega)}{\sqrt{E-2 \omega}-1}\left(\boldsymbol{e}^{*} \cdot \boldsymbol{n}\right)^{2}+\frac{\mathcal{D}_{1}(E-\omega) \mathcal{D}_{1}(E-2 \omega)\left(\boldsymbol{e}^{*} \cdot \boldsymbol{n}\right)^{2}}{(\sqrt{E-2 \omega}-1)^{2}}\right)\right]\right\} .
\end{aligned}
$$

Despite its apparent complexity, $\mathrm{d} \sigma_{B W}^{(1)} / \mathrm{d} \Omega$ is only a combination of simple functions $\mathcal{D}_{1}$ and $\mathcal{D}_{2}$, which are defined in (B.2). Thus, it is even somewhat unexpected that the summed result of high-order PT corrections to the (linear in $F$ ) LOPT photodetachment amplitude up to terms $\sim F^{5}$ can be presented in an accessible analytical form (89). Integrating (89) over $\Omega$, we find the total cross section in the BW approach. Including terms up to order $F^{2}$, the result is 


$$
\begin{aligned}
\sigma_{B W}^{(1)}=\frac{64}{3} \pi^{2} \alpha & \left|\phi_{0}(E)\right|^{2} \frac{|\omega-E|^{3 / 2}}{\omega^{3}} \\
& \times\left\{1-\frac{F^{2}}{\omega^{4}}\left[\frac{2+l^{2}}{5}(\omega-\operatorname{Re} E)+l^{2} \operatorname{Re}\left(\frac{\mathcal{D}_{1}(E-\omega)}{\sqrt{E-2 \omega}-1}\right)\right]\right\} .
\end{aligned}
$$

To obtain the RS results, the (complex) parameter $E=\gamma^{2}-\epsilon$ in (89) and (90) should be expanded in powers of $F^{2}$ (see (B.14) and (B.15)-(B.18)) taking into account also the $E$ dependence of the normalization factor $\phi_{0}$ (see (B.6)-(B.8)). Then the resulting terms up to order $F^{4}$ on the rhs of (89) should be collected. Since the final results are too cumbersome to present here (as they correspond to the fifth order of standard (RS) PT), we present here an explicit form only for the linear in I RS correction to (87):

$$
\begin{aligned}
\Delta \sigma_{1}^{R S}=\frac{8 \alpha}{3 \omega^{7}} & \sqrt{\omega-1}\left\{|\boldsymbol{e} \cdot \boldsymbol{n}|^{2}\left[\sqrt{\omega+1}\left(\omega^{2}-7\right)+14-8 \omega\right]-3|\boldsymbol{e} \cdot \boldsymbol{n}|^{4}(\omega-1)^{2}\right. \\
& +l \operatorname{Re}\left\{(\boldsymbol{e} \cdot \boldsymbol{n})^{2}\right\} \frac{(\omega-1)}{\omega}\left[1+2 \omega(\omega-1)-(\omega-1)^{3 / 2} \sqrt{2 \omega-1}\right] \\
& \left.+l \operatorname{Im}\left\{(\boldsymbol{e} \cdot \boldsymbol{n})^{2}\right\} \frac{(\omega-1)^{2}}{\omega}[\sqrt{2 \omega-1}-\sqrt{\omega-1}]\right\} .
\end{aligned}
$$

The total photodetachment rate including the lowest two orders of RS PT is

$$
\begin{aligned}
\Gamma_{R S}^{(1)}=\frac{4 F^{2}}{3 \omega^{4}}(\omega & -1)^{3 / 2}+\frac{4 F^{4}}{9 \omega^{8}} \sqrt{\omega-1}\left\{\sqrt{\omega+1}\left(\omega^{2}-7\right)-\frac{2}{5}\left(3 \omega^{2}+14 \omega-32\right)\right. \\
& \left.+l^{2} \frac{\omega-1}{\omega}\left[1+\frac{7}{5} \omega(\omega-1)-(\omega-1)^{3 / 2} \sqrt{2 \omega-1}\right]\right\}
\end{aligned}
$$

Note the smoother threshold behaviour, $\sim \sqrt{\omega-E}$, where $E=1$, of $\Delta \sigma_{1}^{R S}$ compared to that of $\sigma_{0}$, which originates from the S-wave part of the detached electron's final-state wavefunction in the third PT order ${ }^{12}$. Thus, the strong field corrections are most important for near-threshold frequencies. The term $\sim l \operatorname{Im}\left\{(\boldsymbol{e} \cdot \boldsymbol{n})^{2}\right\}$ in (89) and (91) describes the ED effect, see (71). We note the considerable threshold suppression of the ED term, $\sim(\omega-1)^{2}$, as well as its smallness at high frequencies (by a factor $\sim \omega^{-1}$ ) compared to the leading terms in (91). This is the reason that the numerical value of the $\operatorname{ED}$ parameter in the $\operatorname{AD} \Gamma^{(1)}(\boldsymbol{n})$ is small (although nonzero) and does not exceed a fraction of one per cent for any frequency $\omega>1$.

Although the field amplitude $F$ is a formally small parameter of the PT analysis, the actual parameter of the PT expansion (87) is $I / \omega^{4}$ (see (89) and (92)). Thus, for $\omega>1$ a few orders of RS or BW PT expansions give a reasonable result for $\mathrm{d} \sigma^{(1)} / \mathrm{d} \Omega$ for even high intensities up to $I=F^{2} \simeq 1$. In figure 26(a) we present the frequency dependence of the total rates $\sigma_{R S}^{(1)}$ and $\sigma_{B W}^{(1)}$ for $F=0.5(I=0.25)$ taking into account the terms of order $I$ and $I^{2}$. The BW result is obtained from (89) with the quasienergy $\epsilon$ calculated from equations (B.7) and (B.9). As mentioned above, the intensity corrections are most important near the threshold, where the BW result is more accurate than the RS one. The curve for $\sigma_{B W}^{(1)}$ in figure 26(a) starts from the dynamical threshold, $\omega_{t h}(F)=-\operatorname{Re} \epsilon$, which is shifted from $\omega_{t h}(0)=1$ because of the (negative) Stark shift. Generally, the RS curve for $\sigma^{(1)}$ starts from the unshifted threshold, $\omega_{t h}(0)$, although the result (92) gives a negative value of $\Gamma^{(1)}$ for $1<\omega<\omega_{t h}(F)$. (These physically senseless results for the decay rate are not presented in figure 26(a).) This fact indicates the breakdown of high-order RS results in the near-threshold domain. Formally, this inaccuracy is caused by the use of power expansions (in $F$ ) of threshold factors reducing (90)

12 The terms of order $F^{2}$ in the BW results (89) and (90) also have a threshold behaviour $\sim \sqrt{\omega-E} \mid$, as may be verified from the threshold behaviour of the $\phi_{0}(E)$ and $\mathcal{D}_{1}(E-\omega)$ functions. 

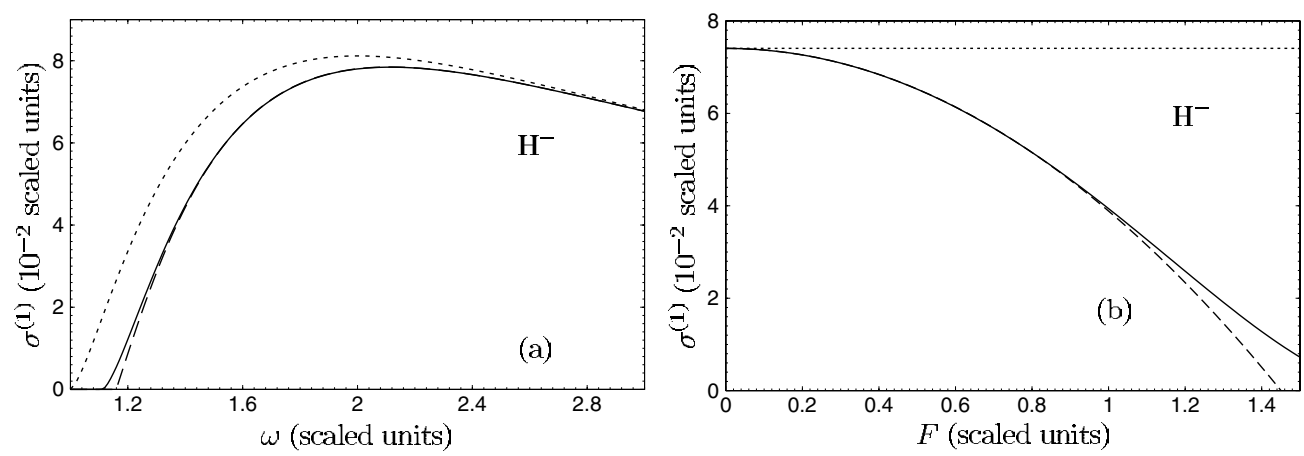

Figure 26. One-photon detachment cross section of $\mathrm{H}^{-}$in a linearly polarized laser field. Short broken curve: the LOPT result, $\sigma_{0}$; full curve: the BW result, $\sigma_{B W}^{(1)}$, taking into account corrections $\sim I$ and $I^{2}$; long broken curve: the RS result, $\sigma_{R S}^{(1)}$, taking into account corrections $\sim I$ and $I^{2}$. (a) Frequency dependence for $F=0.5$. (b) Field dependence for $\omega=1.61$.

to (92). With increasing $\omega$, the intensity corrections become negligible up to $I \sim 1$. The general result of high-order PT corrections is the lowering of the one-photon cross section in a strong field compared with $\sigma_{0}$. This suppression increases with increasing $F$, as is demonstrated in figure 26(b) for a fixed frequency $\omega$. The RS PT breaks down for $F>1$ while the three-term $\mathrm{BW}$ result is correct. Comparison with the exact numerical results shows that terms of order $\sim I^{3}$ and higher change the BW result by less than $2 \%$ up to $F \simeq 1.5$.

\subsection{The quasistationary stabilization regime}

The results of the previous section show that, as $F$ increases, for a high frequency field, the PT regime, in which $\Gamma^{(1)} \sim F^{2}$, evolves smoothly into a 'stabilization-like' behaviour (i.e. the detachment rate decreases). The PT analysis demonstrates clearly the onset of this stabilization behaviour. Moreover, in strong fields, even for high frequencies, $\omega>1$, the contributions of two- (and more) photon detachment channels become important. Thus, in this case the $F$ dependence of the total rate $\Gamma$ should be analysed. In figure 27 we present the results of exact numerical calculations for $\Gamma(F)[170]$ (see also [134, 174]) for different values of $l$ and for $\omega=1.5$, which corresponds to the case of $\mathrm{H}^{-}$irradiated by a $\mathrm{Nd}$ :YAG laser. One sees that the stabilization behaviour breaks down at the closure of the one-photon ionization channel, i.e. at $F=F_{t h}^{(1)}$. One sees also that the threshold structure of $\Gamma(F)$ at higher thresholds $F=F_{t h}^{(n)}$ (corresponding to the closure of higher- $n$ ATD channels with $n \geqslant 2$ ) is significantly different from that for $n=1$ and depends sensitively on the laser polarization. Note that the finite value of $\Gamma$ at $F=F_{t h}^{(1)}$ (where $\Gamma^{(1)}$ vanishes) results from the contributions of partial rates $\Gamma^{(n)}$ for $n$-photon ATD with $n=2,3, \ldots$, whose $F$ dependence (for $n>2$ ) is essentially perturbative for $F \sim F_{t h}^{(1)}$. This feature is rather clear in figure 28, where the partial rate contributions to $\Gamma$ are presented separately. With increasing $F$ in the superstrong field limit, $F \gg 1$, the decay rate increases with no limit (see section 7).

One general comment is necessary concerning the physical interpretation of $\Gamma=-2 \operatorname{Im} \epsilon$ in strong and superstrong field regions (see section 6) when the magnitude of $\Gamma$ can be of order unity, $\Gamma \approx 1$ (see, e.g., figures 27 and 35). Obviously, in such cases the concept of the decay rate becomes inapplicable both formally (since the exponential decay law only applies for $\Gamma \ll|\operatorname{Re} \epsilon|$; see, e.g., [81, 175] for details) and physically (since the idealization of a long 


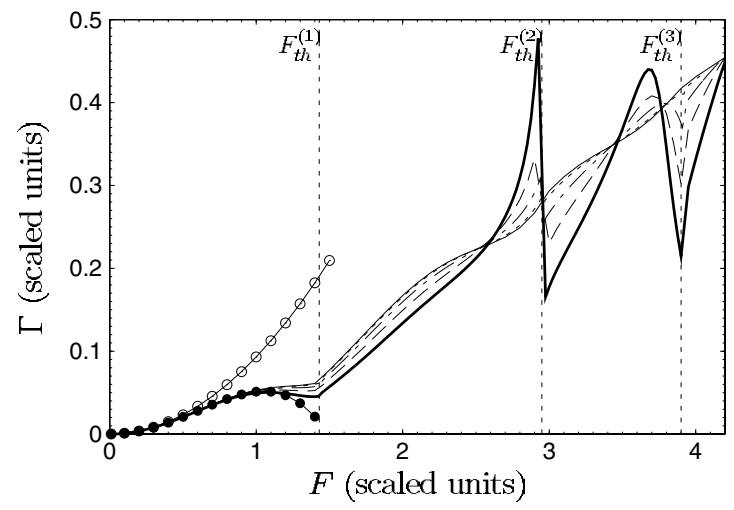

Figure 27. $F$ dependence of the total detachment rate $\Gamma$ for $\omega=1.5$. Thick full curve: $l=0$; thin broken curve: $l=0.22$; thin chain curve: $l=0.47$; thin dotted curve: $l=0.72$; thin full curve: $l=1$. Open circles: the LOPT result for $\Gamma^{(1)} \sim F^{2}$. Full circles: PT result for $\Gamma^{(1)}+\Gamma^{(2)}$ (including terms up to order $F^{4}$ ) for $l=0.72$.

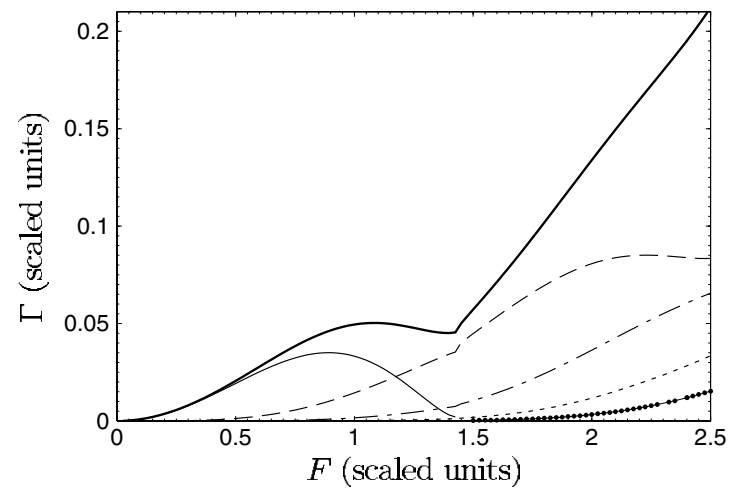

Figure 28. Partial rate contributions $\Gamma^{(n)}$ to the $F$ dependence of $\Gamma$ for $\omega=1.5$ and $\xi=0$. Thin full curve: $\Gamma^{(1)}$; broken curve: $\Gamma^{(2)}$; chain curve: $\Gamma^{(3)}$; dotted curve: $\Gamma^{(4)}$; full curve with full circles: $\Gamma^{(5)}$; thick full curve: $\Gamma$.

monochromatic laser pulse fails if the ionization time is of the order of the atomic period). Moreover, in reality superstrong fields are produced in the form of short pulses; thus the constant laser amplitude $F$ is changed to that of a pulse envelope, $F \rightarrow F_{\text {max }} f(t)$ (with $F_{\text {max }}$ its peak value and $f(t)$ its temporal shape function), and the total ionization probability at the end of a pulse, $W_{t o t}$, measures the experimental ionization or detachment yield. Although the correct calculation of $W_{\text {tot }}$ requires a numerical solution of the TDSE, a reasonable approximation for $W_{\text {tot }}$ gives an adiabatic result

$$
W_{t o t}^{(a d)}=1-\exp \left[-\int_{-\infty}^{\infty} \Gamma\left(F_{\max } f(t)\right) \mathrm{d} t\right],
$$

based on the instantaneous decay rates $\Gamma\left(F_{\max } f(t)\right)$. As shown in $[160,163,176]$, the close agreement of exact results for $W_{t o t}$ with $W_{t o t}^{(a d)}$ extends well into the DS regime, e.g. for H(1s) up to 5 cycle pulses with $F_{\max } \leqslant 10$ au [160]. This means that DS originates from QS of decay rates $\Gamma$, and that even for strong, short pulse fields, the QQES approach does not lose its significance, thereby providing a convenient adiabatic basis. 

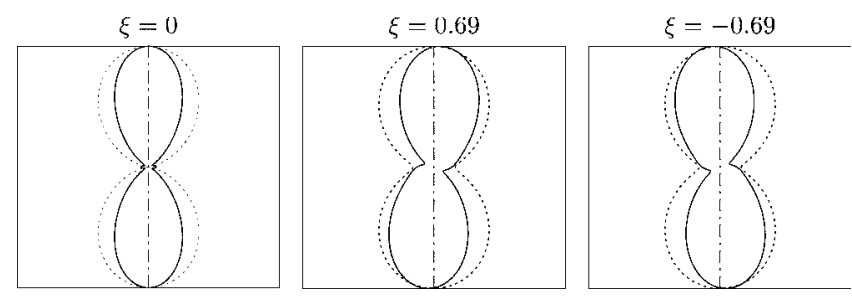

Figure 29. Ellipticity dependence of the total $\mathrm{AD}, \Gamma(\boldsymbol{n})$, at $\omega=1.5$ and $F=1.35$ (in the plane of laser polarization). Dotted curves: LOPT result for $n=1$.

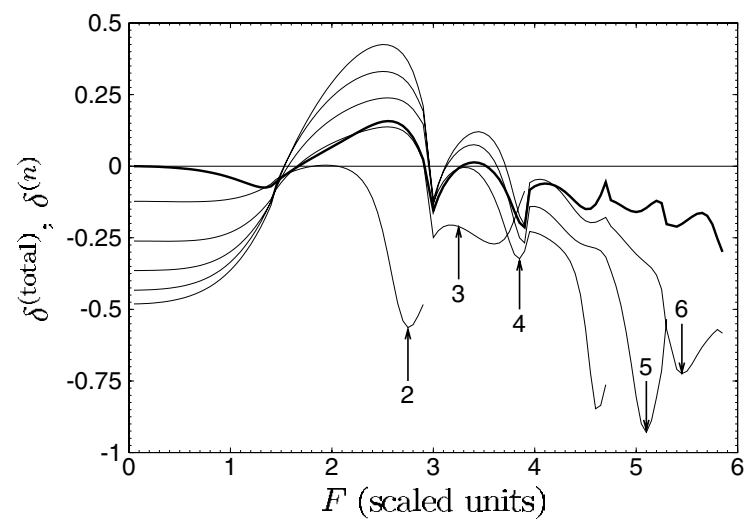

Figure 30. Same as figure 24 but for $\omega=1.5$.

\subsection{Angular distributions and ED for $\omega>1$}

The total (summed over $n$ ) $\mathrm{AD}, \Gamma(\boldsymbol{n})$, in the stabilization and 'post-stabilization' regions is rather simple. In figure 29 we present $\Gamma(\boldsymbol{n})$ for $\omega=1.5$ and for $F=1.35$ that lies in the stabilization interval, $F<F_{t h}^{(1)}$, close to $F_{t h}^{(1)}$. For linear polarization, strong field effects result in a narrowing of the ADs as compared to the LOPT result for $n=1$. Similarly, for $F_{t h}^{(1)}<F<F_{t h}^{(2)}$, the dependence of $\Gamma(\boldsymbol{n})$ on $F$ (not shown) is largely due to $\Gamma_{\text {lopt }}^{(2)}(\boldsymbol{n})$, both for $\xi=0$ and for \pm 0.69 . Consequently, the $\mathrm{AD}$ in this region is similar to that shown in figure 29, since there also $\Gamma(\boldsymbol{n})$ is dominated by the contribution of $\Gamma^{(2)}(\boldsymbol{n})$ since the $n=1$ threshold is closing. Note the signature of ED in figure 29: although for $\Gamma^{(1)}(\boldsymbol{n})$ the ED parameter is small up to $F \approx F_{t h}^{(1)}$, in figure 29 the ED originates primarily from the two-photon ATD channel, $\Gamma^{(2)}(\boldsymbol{n})$, whose contribution, as just noted, is dominant at $F \approx F_{t h}^{(1)}$. The ED parameters $\delta^{(n)}$ and $\delta^{(t o t a l)}$ for $\omega=1.5$ are presented in figure 30 over a wide interval of intensities. Concerning the strong field behaviour of the ED parameters (when a few of the ATD channels are closed), the same arguments as for the results in figure 25(b) for $\omega=0.8$ are valid: the increasing trend (in absolute value) of $\delta^{(n)}$ and $\delta^{(t o t a l)}$ with increasing $F$ is caused completely by the increase of $\operatorname{Im} \epsilon$ (or, equivalently, by the total rate $\Gamma$ ) in the superstrong field limit. As will be discussed in the next section, in this limit the dependence of $\Gamma$ on $F$ exhibits a universal behaviour that is largely independent of the frequency. 


\section{Static-electric-field behaviour of decay rates in superstrong fields}

\subsection{General remarks}

The accurate description of the intensity dependence of the decay rate of a bound level over a broad interval of laser frequencies is one of the challenging problems of strong field laser-atom physics. Existing qualitative results obtained from nonperturbative (in the intensity) analyses of atomic decay rates depend significantly on the relation between the laser frequency $\omega$ and $\omega_{0}=1\left(=\left|E_{0}\right| / \hbar\right.$ in absolute units $)$, as well as on the relation between the laser amplitude $F$ and $F_{0}=1\left(=\sqrt{2 m\left|E_{0}\right|^{3}} /|e| \hbar\right.$ in absolute units $)$. For small frequencies, $\omega \ll 1$, and field strengths $F \geqslant \omega$, or equivalently for $\gamma<1$, the tunnelling regime for the decay is realized, which is applicable only for weak (although nonperturbative) fields, $F \ll 1$. For the case of ground-state atomic hydrogen, $\mathrm{H}(1 \mathrm{~s})$, Pont et al $[177,178]$ performed a low frequency analysis of the decay rate $\Gamma$ beyond the KA (up to $F \leqslant 0.2$ ) using the $\omega^{2}$ expansion of the complex quasienergy within a basis of quasistationary states of the hydrogen atom in a static electric field (whose magnitude was equal to that of the instantaneous laser field, see below). For $\omega=0.134(\lambda=616 \mathrm{~nm})$, the comparison of the $F$ dependence of these 'staticfield-based' results with the exact ones shows a reasonable agreement (which becomes better for stronger $F$ ) except for the structure seen in the exact $\Gamma(F)$, which is due to Rydberg levels shifting in and out of resonance as the intensity varies. With increasing $F$ (e.g. for $F \geqslant 0.2$ in the case of $\mathrm{H}(1 \mathrm{~s})$ ), over-barrier ionization becomes important. Recently, the overbarrier decay rate $\Gamma$ in the low frequency limit, $\omega \ll 1$, has been obtained by Popov [39] by an adiabatic cycle-averaging of the Stark width $\Gamma_{\text {stat }}$ for a strong static electric field. It demonstrates a surprisingly linear dependence of $\Gamma$ on $F$ (the 'intermediate' asymptotic regime [39]):

$$
\Gamma \approx k\left(F-F_{c r}\right)
$$

where the fitting parameters $k$ and $F_{c r}$ do not depend on $F$ over a wide fitting interval (e.g. $0.6<F<2$ for $\mathrm{H}(1 s)$ ) and are smooth functions of the laser ellipticity. For abovethreshold frequencies, $\omega>1$, and in the strongly nonperturbative regime, the concept of QS of atomic decay rates is conventionally understood to be applicable, in which case $\Gamma(F)$ has a decreasing trend with increasing $F$ (see section 5). However, for a Coulomb potential the existence of a stabilization regime for decay rates in the ultrastrong field limit is still an open question. Moreover, for a short-range potential, as discussed previously in section 5.2, a stabilization-like behaviour exists only over a limited interval of intensity, after which $\Gamma$ tends to increase without limit with increasing $F$ (on average, with fluctuations due to TAs).

In order to show the general features of $\Gamma(F, \omega)$ over broad intervals of $F$ and $\omega$, in figure 31 we present exact numerical results for $\Gamma(F, \omega)$ for the case that $l=0.72$ for frequencies in the interval $0.15<\omega<2$ and for four different values of $F$ between $F=0.01$ (PT region) and $F=1.0$ (strong field regime) [170]. For weak $F, \Gamma(\omega)$ exhibits typical perturbative behaviour, i.e. a step-like increase as $\omega$ increases that results from the sequential contributions of the $n$-photon partial rates, $\Gamma^{(n)} \sim F^{2 n}$, with $n_{0}$ (the minimum number of photons necessary for ionization) becoming smaller as $\omega$ increases. As $F$ increases, the stair-step behaviour gradually disappears as $\Gamma(\omega)$ becomes nearly insensitive to $\omega$ for essentially nonperturbative values of $F$. This unusual behaviour of $\Gamma(\omega)$ at high $F$ allows us to assume that, in the strong field limit, the decay mechanism itself becomes essentially independent of the frequency, even in the $\omega>1$ domain. In the next section we present the analytical basis for this result. 


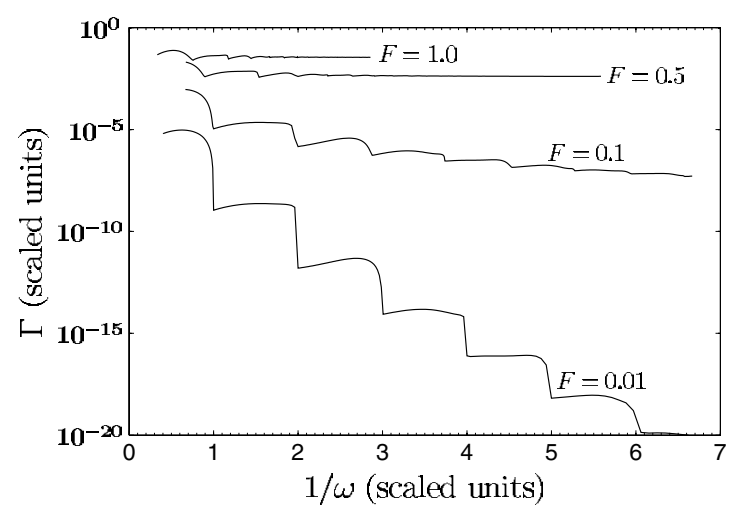

Figure 31. $1 / \omega$ dependence of the total detachment rate $\Gamma$ for four values of $F$, as indicated in the figure, and for $l=0.72$.

\section{2. $\omega^{2}$ expansion for the complex quasienergy}

To analyse the strong field regime in more detail, instead of the conventional representation (11) for a quasienergy state, we use the following one:

$$
\Psi_{\epsilon}(\boldsymbol{r}, t)=\chi(\boldsymbol{r}, t) \exp \left(-\mathrm{i} \int^{t} \mathcal{E}\left(t^{\prime}\right) \mathrm{d} t^{\prime}\right),
$$

where $\Psi_{\epsilon}(\boldsymbol{r}, t)$ is the solution of the Schrödinger equation for a Hamiltonian $H(\boldsymbol{r}, t)=$ $H_{a t}(\boldsymbol{r})+V(\boldsymbol{r}, t)$, where $H_{a t}(\boldsymbol{r})$ describes the atom and $V(\boldsymbol{r}, t)=\boldsymbol{r} \cdot \boldsymbol{F}(\omega t)$. The periodic functions $\chi(r, t)$ and $\mathcal{E}(t)$ satisfy the following equation:

$$
\left(H_{a t}(\boldsymbol{r})+\boldsymbol{r} \cdot \boldsymbol{F}(\omega t)-\mathcal{E}(t)-\mathrm{i} \frac{\partial}{\partial t}\right) \chi(\boldsymbol{r}, t)=0 .
$$

Since $\Phi_{\epsilon}, \chi$ and $\mathcal{E}(t)$ are periodic in time, the quasienergy $\epsilon$ is the cycle average of $\mathcal{E}(t)$ :

$$
\epsilon=\frac{1}{T} \int_{0}^{T} \mathcal{E}(t) \mathrm{d} t, \quad \text { where } T=2 \pi / \omega .
$$

Equations (95)-(97) are very general and were used by Langhoff et al [172] in their analyses of so-called 'secular terms' in higher orders of PT (in $V$ ) and by Pont et al [178] in the low frequency analysis of the ionization of $\mathrm{H}(1 \mathrm{~s})$. In [178] the formal development of a PT in $W=-\mathrm{i} \omega \partial / \partial \tau$, where $\tau=\omega t$, is presented for calculations of $\chi(r, t)$ and $\mathcal{E}(t)$ based on the instantaneous state, $\chi^{(0)}(\boldsymbol{r}, t)$, with energy $\mathcal{E}^{(0)}(t)$, of an atom in a static electric field of strength $\mathcal{F}=|\boldsymbol{F}(\omega t)|$. In what follows, we employ such an approach to analyse the frequency dependence of $\epsilon$ for the $\delta$-model potential in the strong field limit [170]. Since we do not assume that $\omega$ is small compared to the binding energy $\left|E_{0}\right|$, the key issue is to calculate the next order correction, $\mathcal{E}^{(2)}(t) \sim \omega^{2}$, to $\mathcal{E}^{(0)}(t)$ in order to estimate the accuracy of the expansion of $\epsilon$ in a power series in $\omega^{2}$, which generally is an asymptotic expansion in $F$ [178]. Note that in [178] it is shown that $\mathcal{E}^{(1)}(t)=0$. Also, corresponding to $\mathcal{E}^{(n)}(t)$, we define $\epsilon^{(n)}$ according to equation (97).

The general result for $\mathcal{E}^{(2)}(t)$ is [178]

$$
\mathcal{E}^{(2)}(t)=\omega^{2}\left\langle\frac{\partial \tilde{\chi}^{(0)}(\boldsymbol{r}, t)}{\partial \tau}\left|\mathcal{G}_{\mathcal{E}^{(0)}(t)}^{\prime}\left(\boldsymbol{r}, \boldsymbol{r}^{\prime}\right)\right| \frac{\partial \chi^{(0)}\left(\boldsymbol{r}^{\prime}, t\right)}{\partial \tau}\right\rangle,
$$

where $\mathcal{G}_{\mathcal{E}^{(0)}(t)}^{\prime}\left(\boldsymbol{r}, \boldsymbol{r}^{\prime}\right)$ is the reduced Green function of an atom in a static electric field and $\tilde{\chi}^{(0)}(\boldsymbol{r}, t)$ is the 'dual' function, $\tilde{\chi}^{(0)}(\boldsymbol{r}, t)=\chi^{(0) *}(\boldsymbol{r},-t ;-\xi)$, which is necessary to provide 

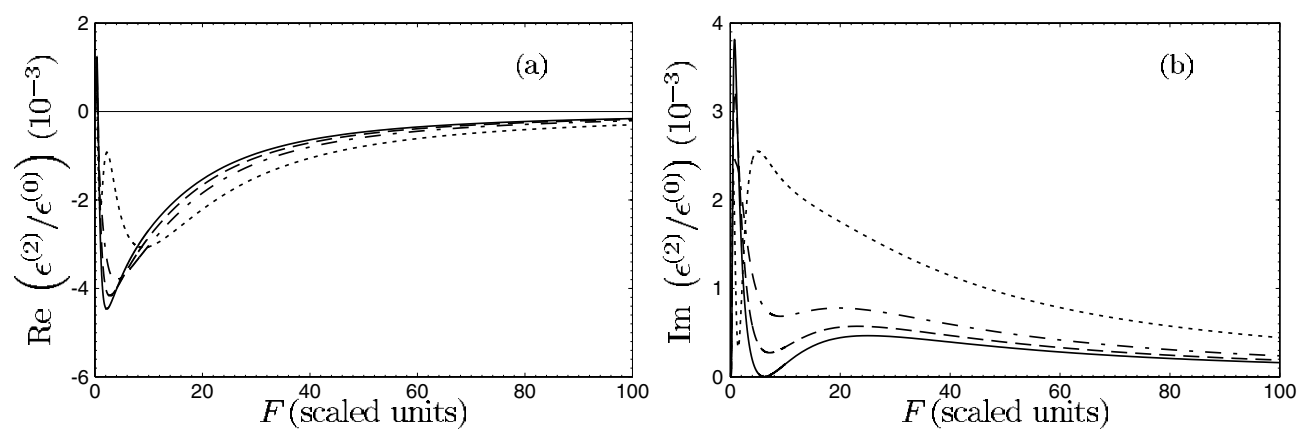

Figure 32. Dependence on $F$ and $l$ of the real (a) and imaginary (b) parts of the ratio $\epsilon^{(2)} / \epsilon^{(0)}$ for $\omega=1$. Full curve: $l=0$. Long broken curve: $l=0.5$. Chain curve: $l=0.7$. Short broken curve: $l=0.9$.

a proper normalization of the quasistationary (resonance) state $\chi^{(0)}(r, t)$, see (19). In the $\delta$-model potential (see the review [29] for details), $\mathcal{E}^{(0)}(t)$ at any fixed $t$ can be obtained as the root of the transcendental equation:

$$
1+\pi \mathcal{F}^{1 / 3} J(\xi)=0
$$

where $\xi=-\mathcal{E}^{(0)}(t) \mathcal{F}^{-2 / 3}, \mathcal{F} \equiv|\boldsymbol{F}(\omega t)|=F \sqrt{(1+l \cos 2 \omega t) / 2}$, and $J(\xi)$ is a combination of regular (Ai) and irregular (Bi) Airy functions and their derivatives:

$$
J(\xi)=\operatorname{Ai}^{\prime}(\xi) \operatorname{Bi}^{\prime}(\xi)-\xi \operatorname{Ai}(\xi) \operatorname{Bi}(\xi)+i\left[\operatorname{Ai}^{\prime 2}(\xi)-\xi \operatorname{Ai}^{2}(\xi)\right] .
$$

Using the explicit forms of $\chi_{0}(\boldsymbol{r}, t)$ and $\mathcal{G}_{\mathcal{E}^{(0)}(t)}^{\prime}\left(\boldsymbol{r}, \boldsymbol{r}^{\prime}\right)$, the matrix element in (98) can be calculated analytically in terms of Airy functions and their derivatives [170]. The general result simplifies for the case of a circularly polarized laser field $(l=0)$. In this case $\mathcal{E}(t)$ is time-independent, $\epsilon_{\text {circ }}^{(0)}=\mathcal{E}^{(0)}$ and the correction $\epsilon_{\text {circ }}^{(2)} \equiv \mathcal{E}^{(2)}$ is $[134,170]$

$$
\epsilon_{\text {circ }}^{(2)}=\frac{\omega^{2}}{360(F / \sqrt{2})^{2 / 3}} \frac{J^{(5)}\left(\xi_{0}\right)}{J^{(1)}\left(\xi_{0}\right)},
$$

where $\xi_{0}=\epsilon_{\text {circ }}^{(0)}(F / \sqrt{2})^{-2 / 3}$ and $J^{(n)}(x)=\mathrm{d}^{n} J(x) / \mathrm{d} x^{n}$. In the weak field limit $(F \ll 1)$, neglecting exponentially small (tunnelling) terms, the following result for $\epsilon=\epsilon^{(0)}+\epsilon^{(2)}$ for an elliptic polarization is valid [170]:

$$
\epsilon=-1-\frac{F^{2}}{32}\left\{1+\frac{3 F^{2}}{4}\left(1+\frac{l^{2}}{2}\right)+\frac{7}{24} \omega^{2}\left[1+\frac{13}{2} F^{2}\left(1+\frac{25}{28} l^{2}\right)\right]\right\} .
$$

This result coincides exactly with the first two terms in the $\omega$ expansion of the PT result (B.14) for $\epsilon$ taking into account the explicit forms (B.15) and (B.16) for the dynamic polarizability and hyperpolarizability. Thus, for weak fields, the 'zero approximation' $\epsilon \simeq \epsilon^{(0)}$, is valid only in the low frequency limit, $\omega \ll 1$. The asymptotic analysis of $\epsilon_{\text {circ }}^{(2)}$ shows that, in ultrastrong fields, $F \gg 1$, the ratio of $\epsilon_{\text {circ }}^{(2)}$ to $\epsilon_{\text {circ }}^{(0)}$ is of order $\left(\omega / F^{5 / 3}\right)^{2}$ [134]. Thus the correction $\epsilon_{\text {circ }}^{(2)}$ is negligibly small at any (finite) frequency. To establish the accuracy of the term $\epsilon^{(0)}$ in the strong field regime for an arbitrary polarization, in figure 32 we present numerical results for real and imaginary parts of the ratio of $\epsilon^{(2)}$ to $\epsilon^{(0)}$ for a number of values of $l$ at fixed $\omega=1$. (Obviously, for other $\omega$ the results scale as $\omega^{2}$.) One observes that, with increasing $F$, the two-term approximation, $\epsilon^{(0)}+\epsilon^{(2)}$ (which we call the adiabatic approximation (AA) result), is applicable over a wide interval of $\omega$, including the above-threshold region, $\omega>1$. 

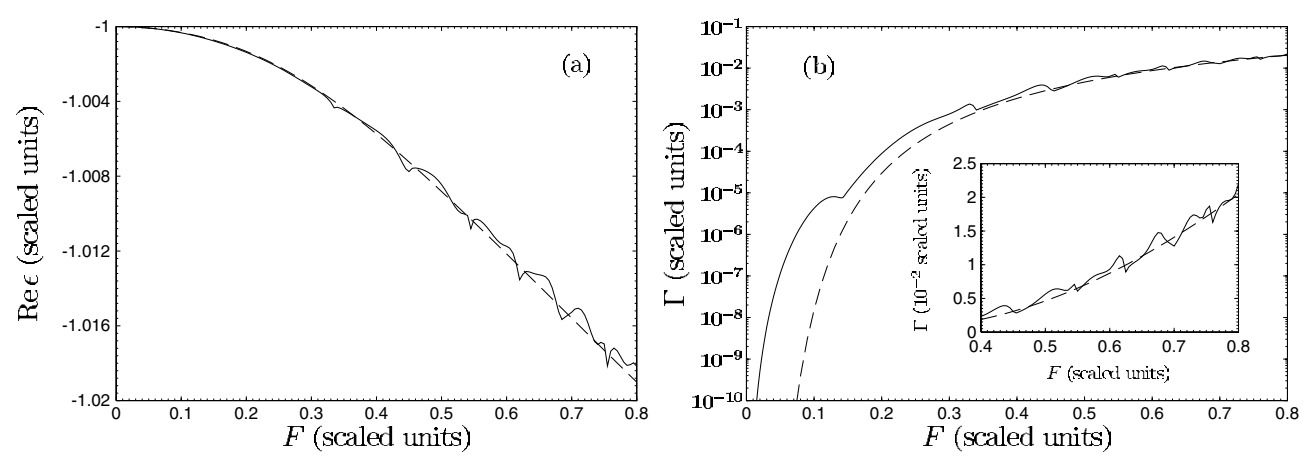

Figure 33. $F$ dependence of the real (a) and imaginary (b) parts of the complex quasienergy for $\omega=0.36$ and $l=1$. Full curve: the exact QQES result. Broken curve: the AA result.
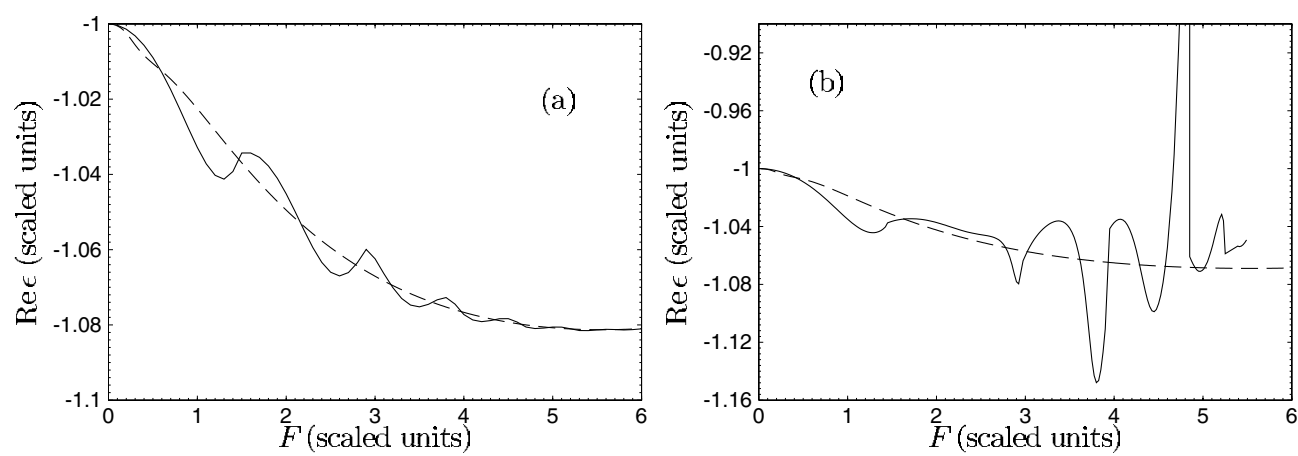

Figure 34. $F$ dependence of $\operatorname{Re} \epsilon$ for $\omega=1.5$ : (a) $l=0$ and (b) $l=1$. Full curve: the exact QQES result. Broken curve: the AA result.

\subsection{Comparisons with numerical results}

To check both the relation between the AA results and exact numerical results for $\epsilon$ and also the applicability of the $\delta$-potential model for real negative ions in a strong laser field, in table 4 we compare our numerical (QQES) and approximate (AA) results for the detachment of $\mathrm{H}^{-}$ by linearly polarized $\mathrm{CO}_{2}$ laser radiation (for which $\omega=0.155$ ) with existing theoretical predictions [97, 123, 124, 128]. As noted below equation (9), our scaled unit of intensity for $\mathrm{H}^{-}$is $I_{0}=1.498 \times 10^{12} \mathrm{~W} \mathrm{~cm}^{-2}$. The comparisons in table 4 show the excellent agreement of our exact QQES results with more refined (and time-consuming) calculations and also the high accuracy of the AA results for nonperturbative intensities $I \geqslant 5 \times 10^{10} \mathrm{~W} \mathrm{~cm}^{-2}$, when $\gamma \leqslant 1$.

Comparisons of $\epsilon_{Q Q E S}$ and $\epsilon_{A A}$ as functions of $F$ are presented in figure 33 for $\omega<1$ and in figures 34 and 35 for $\omega>1$. The AA and QQES results for $l=0$ and $\omega<1$ are almost indistinguishable: for $\omega=0.36$ and $F>0.3$ the difference between $\epsilon_{Q Q E S}$ and $\epsilon_{A A}$ is less than 3\%; for $\omega=0.56$ and $F>0.4$ it is less than $2 \%$; and for $\omega=0.77$ and $F>0.5$ the difference is less than $4 \%$. Generally, the AA results describe accurately the trends of the position and the width of a quasistationary level but fail to describe the thresholdrelated peculiarities, which are lost by using the $\omega^{2}$ expansion for the iterative solution of equation (96). These peculiarities are most pronounced for the case of linear polarization and they are exhibited at the points of non-analyticity of the function $\epsilon(F)$, which correspond 

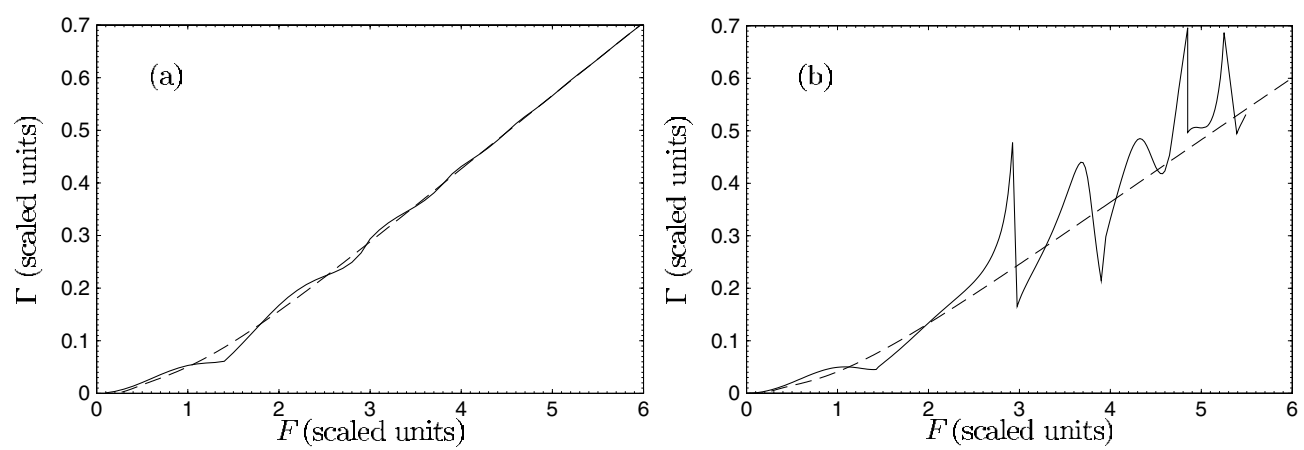

Figure 35. $F$ dependence of $\Gamma$ for $\omega=1.5$ : (a) $l=0$ and (b) $l=1$. Full curve: the exact QQES result. Broken curve: the AA result.

Table 4. Detachment rates for $\mathrm{H}^{-}$in the field of a $\mathrm{CO}_{2}$ laser having linear polarization.

\begin{tabular}{|c|c|c|c|c|c|c|}
\hline \multirow{2}{*}{$\begin{array}{l}\text { Intensity }^{\mathrm{a}} \\
\left(\mathrm{W} \mathrm{cm}{ }^{-2}\right)\end{array}$} & \multicolumn{6}{|c|}{ Detachment rates $^{\mathrm{a}}(\mathrm{au})$} \\
\hline & [97] & [124] & [128] & [123] & AA & QQES \\
\hline $1.0(10)$ & & $0.97(-9)$ & $0.91(-9)$ & $(1.04 \pm 0.12)(-9)$ & $0.32(-9)$ & $0.97(-9)$ \\
\hline $1.12(10)$ & $\begin{array}{l}2.7(-9)^{\mathrm{b}} \\
2.1(-9)^{\mathrm{c}}\end{array}$ & & & $(2.04 \pm 0.11)(-9)$ & $0.73(-9)$ & $2.28(-9)$ \\
\hline $2.52(10)$ & $\begin{array}{l}1.4(-7)^{\mathrm{b}} \\
1.0(-7)^{\mathrm{c}}\end{array}$ & & & $(1.12 \pm 0.08)(-7)$ & $0.88(-7)$ & $1.14(-7)$ \\
\hline $5.0(10)$ & & $1.67(-6)$ & $1.76(-6)$ & $(1.81 \pm 0.06)(-6)$ & $1.64(-6)$ & $1.79(-6)$ \\
\hline $1.0(11)$ & & $1.61(-5)$ & $1.61(-5)$ & $(1.68 \pm 0.03)(-5)$ & $1.62(-5)$ & $1.66(-5)$ \\
\hline $1.6(11)$ & & & & $(5.91 \pm 0.02)(-5)$ & $5.74(-5)$ & $6.12(-5)$ \\
\hline $2.0(11)$ & & & & $(9.97 \pm 0.01)(-5)$ & $9.75(-5)$ & $9.87(-5)$ \\
\hline
\end{tabular}

a $(n) \equiv 10^{n}$

${ }^{\mathrm{b}}$ Floquet calculations with a parametrized one-electron potential.

${ }^{\mathrm{c}}$ Faisal-Reiss formulae with a Hylleraas ground-state wavefunction.

to the closure of partial ATD channels with increasing $F$ (at $F=F_{t h}^{(n)}$ ). As we discussed above, these points are branch points of the type $\left(\epsilon+u_{p}+n \omega\right)^{k+1 / 2}$ and as $F$ increases (and $\operatorname{Im} \epsilon$ becomes important) they are shifted to the complex $F$ plane. Thus, in strong fields the peculiarities of $\epsilon(F)$ on the real $F$ axis become more smooth. As figures 33-35 demonstrate, in the strong-field limit, the behaviour of the exact results for $\epsilon(F)$ (when averaged over the threshold peculiarities) show surprisingly close coincidence with the AA results, even in the high frequency domain, $\omega>1$. Moreover, over a wide interval of $F$ the $F$ dependence of $\Gamma$ (averaged over threshold peculiarities) is close to linear, which is similar to the 'intermediate' asymptotic result (94) found for $\mathrm{H}(1 \mathrm{~s})$ in the low frequency limit. For instance, at $\omega=\omega_{\mathrm{CO}_{2}}$ (see table 4), the parameters for this linear dependence are $F_{c r}=0.86$ and $k=0.12$ for $l=1$, and results obtained from formula (94) are in reasonable agreement with the exact ones beginning from $F>1.5$ (or for $I>2.25 \times 10^{12} \mathrm{~W} \mathrm{~cm}^{-2}$ for $\mathrm{H}^{-}$). Unlike the adiabatic case $(\omega \ll 1)$, for a finite frequency the interval of applicability of the asymptotic form (94) depends on $\omega$ : as $\omega$ increases, the result (94) becomes applicable at stronger fields. Namely, for $\omega=1.5$ (when the parameters $k$ and $F_{c r}$ in (94) are $F_{c r}=0.84, k=0.13$ for $l=0$, and $F_{c r}=0.89, k=0.1165$ for $\left.l=1\right)$ the linear in $F$ regime is realized with an accuracy of about $5 \%$ over the interval $2.5<F<10$. 
The results presented in this section justify the conceptual statement, namely that the decay of a weakly bound atomic system in a strong laser field $\boldsymbol{F}(\omega t)$ with any frequency and polarization state may be described by cycle-averaging the results for an instantaneous static electric field of strength $|\boldsymbol{F}(\omega t)|$.

\section{Summary and conclusions}

Even for long (monochromatic) laser pulses with a shaped focus, the output of a laseratom interaction depends on many parameters, e.g. for multiphoton detachment there are the frequency, intensity and polarization state of a laser as well as the momentum direction of the escaping electron. Since time-consuming, completely numerical $a b$ initio calculations may be performed only for limited sets of such control parameters, simple, analytically solvable models that cover the entire parameter space are particularly useful for determining the best way to control laser-atom processes. For strong laser-atom problems (where ionization strongly affects the features of any laser-atom process), the most important requirement for such models is the existence of both discrete and continuum spectra for the model system in the absence of the laser field. One of the most basic models for laser-atom physics was suggested by Keldysh, namely a single-electron bound state that does not interact with a free-electron continuum (which evolves to a 'Volkov continuum' for nonzero laser field). Thus, in the KA, the coupling between bound and continuum states is provided only by the initial laser excitation of the electron out of the bound state (to which it never returns). It is somewhat surprising that this simplest model allows one to predict qualitative features for a number of laser-atom effects. The major deficiency of the KA is its complete neglect of coupling between bound and continuum states, i.e. of atomic dynamics, since these states are eigenstates of different Hamiltonians.

In this paper we have reviewed (and extended) existing results for a next-level model (compared to the Keldysh one), i.e. the zero-range (or $\delta$-function) potential model system. In the absence of the laser field, this model again involves only a single-electron bound state; however, this state is 'minimally' related to the three-dimensional continuum through the S-wave scattering phase. Nevertheless, for a nonzero laser field, even this 'minimal coupling' allows an accurate, self-consistent account for some major corrections to the Keldysh model, as it includes 'multiple interactions' of the escaping (or quasibound) electron with the binding potential. The (single) bound and continuum states of the $\delta$-model potential form a complete set of eigenfunctions of the unperturbed Hamiltonian, as required for a self-consistent dynamical model. Owing to the fact that a zero-range potential acts only at $r=0$, and thus amounts to a boundary condition on the wavefunction of the system, this model provides the possibility for an accurate quantum treatment of strong laser and binding potential effects on an equal footing. In this review, we have solved this model problem using the QQES approach.

We have formulated the basic results of the QQES approach for a $\delta$-model potential and presented general equations for the multiphoton detachment amplitude in sections 2.3-2.5 and appendices A-C. In particular, we have analysed the key ingredients of this approach, the Fourier coefficients of the QQES wavefunction at the origin, which provide information on binding potential effects in the presence of a laser field. These results may be useful in different applications of this model to describe the behaviour of a shallow bound level subjected to a strong monochromatic perturbation. In this review they have been used in our general analysis of ADs and photon polarization effects in multiphoton detachment of a weakly bound electron. Based on analytical results for $n$-photon ADs of detached electrons obtained both in BW (i.e. using the exact complex quasienergy) and RS versions of PT expansions, the 
ellipticity dependence of ADs and ED effects have been analysed in detail and illustrative examples were presented for photon numbers up to $n=8$. For the nonperturbative regime, numerical results were presented which show the strong field modifications of ADs and dichroic effects. These modifications are most important near the closure of open photodetachment channels.

We have also performed a detailed analysis of the case when the laser frequency exceeds the electron binding energy $\left|E_{0}\right|$, which is important for the description of negative ions in optical and VUV laser fields. In applying our results to multiphoton detachment of $\mathrm{H}^{-}$, satisfactory agreement with existing experiments as well as with more sophisticated calculations that include correlation effects has been found both in the perturbative and in the nonperturbative regimes. A most interesting feature shown in our general analysis is the existence of a stabilization-like behaviour of the total rate $\Gamma$ with increasing $F$ up to the closure of the onephoton photodetachment channel at $F=F_{t h}^{(1)}$. A general analysis for the $F$ dependence of the complex quasienergy $\epsilon$ and the total rate $\Gamma$ in the superstrong field limit (when a number of the lowest ATD channels are closed) shows that the exact results for $\epsilon$ are fairly well approximated by the cycle-averaged complex energy of the quasistationary state in an instantaneous static electric field of strength $|\boldsymbol{F}(\omega t)|$. Furthermore, in the superstrong field regime, $F \gg \omega$, this result is valid not only for $\omega \ll 1$ (as may be expected from adiabaticity arguments) but also for any $\omega$, including the 'post-stabilization' regime $F \gg F_{t h}^{(1)}$ for $\omega>1$. Of course, our proof of this conceptual result is valid only for a short-range potential; its verification for Coulomb-like potentials remains a challenging problem.

Finally, we conclude by noting the efficacy of using the QQES approach for a $\delta$-model potential to provide a qualitative description of recently observed strong laser field effects in real atoms, namely the plateau structures and threshold phenomena in both ATI and HHG processes. For example, an approximate account of binding potential effects (equivalent to the use of approximation (B.32) for the coefficients $f_{n}$ ) has permitted the estimation of the cut-off energy, $\sim 10 U_{p}$, of the ATI plateau [133] (see also [179]). Also, a resonancelike enhancement of ATI peak intensities has been discovered experimentally [180, 181] and confirmed by direct numerical integration of the TDSE [182]. Most recently, the role of channel closings, i.e. threshold effects, have been identified as important for describing the observed enhancement of ATI peaks [183]. The improved KA [133] reproduces the resonant-like behaviour of peaks along the high energy plateau near multiphoton channel closings [183, 184] (see also the quasiclassical analysis in [185]). A rigorous analytical and numerical analysis employing the exact QQES results (36) and (38) [135] has shown that the enhancements mentioned above have a purely quantum origin that stems from well-known threshold phenomena (TAs) in multichannel problems (see, e.g., [142, 148, 186, 187]). Similar TAs in the region of the HHG plateau have been shown to induce significant enhancements of the HHG spectrum, as discussed in [136]. Based upon these past successes, we conclude that the application of this model to the description of laser-atom and laser-negative ion processes holds great promise for elucidating known phenomena and for predicting new phenomena (see, e.g., [137]). In particular, the plateau structures and threshold phenomena predicted by our simple short-range potential model may be expected to be observable in negative ions as well, using strong infrared laser radiation.

\section{Acknowledgments}

This work has been supported in part by the CRDF and the RF Ministry of Education under grant no VZ-010-0, by grant E02-3.1-115 of the RF Ministry of Education (NLM), and by the US National Science Foundation under grant no PHY-0070980. 


\section{Appendix A. Normalization of the QQES wavefunction for a $\delta$-model potential}

For the $\delta$-model potential, the definition of the dual function in (19) is equivalent to the following alternative definition ( $\mathrm{cf}(21))$ :

$$
\tilde{\Phi}_{\epsilon}(\boldsymbol{r}, t)=-4 \pi \int_{-\infty}^{0} \mathrm{e}^{\mathrm{i} \epsilon^{*} \tau} G^{(-)}(\boldsymbol{r}, t, 0, t-\tau) \tilde{f}_{\epsilon}(t-\tau) \mathrm{d} \tau
$$

where $G^{(-)}(r, t, 0, t-\tau)$ is the advanced Green function (14) and the integral over $\tau$ is considered as analytically continued from the lower half-plane of complex $\epsilon$. Taking into account (23) and integrating over $\boldsymbol{r}$ and $t$, the relation (18) gives

$2 \sqrt{\pi \mathrm{i}} \sum_{n, k} \mathrm{i}^{n-k} \tilde{\phi}_{n}^{*} \phi_{k} \int_{0}^{\infty} \frac{\mathrm{d} \tau}{\tau^{1 / 2}} \exp [-\mathrm{i}((E-n \omega-k \omega) \tau-z(\tau))] J_{k-n}(z(\tau))=1$,

where the integral over $\tau$ is calculated by differentiating $M_{0, k}(E)$ in (29) with respect to $E$. As a result, the normalization condition (18) may be expressed in terms of the same matrix elements that enter the basic equations (27) and (28) for $\phi_{n}$ and $\epsilon$ :

$$
\left.4 \pi \sum_{n, k=-\infty}^{\infty} \tilde{\phi}_{n}^{*} \phi_{k} \frac{\partial}{\partial \mathcal{E}}\left[\sqrt{\mathcal{E}-2 n \omega} \delta_{n, k}-M_{n, k}(\mathcal{E})\right]\right|_{\mathcal{E}=E}=1 .
$$

In fact, for the case considered, the normalization procedure serves to define the coefficient $\phi_{0}$, since $\phi_{n \neq 0}$ can be expressed in terms of $\phi_{0}$ by means of the homogeneous equations (27). Thus, the identity (A.3) may be considered as an equation for the product $\tilde{\phi}_{0}^{*} \phi_{0}$. The symmetry properties for the coefficients $\phi_{n}$ and $\tilde{\phi}_{n}$ may be deduced from the following considerations: the matrix elements (29) are independent of the sign of $\xi$, and the relation (A.3) is an even function of $\omega$. Also, the system of linear equations for $\tilde{\phi}_{n}^{*}$, is similar to (27) (with complex-conjugated matrix elements). Thus, one determines that

$$
\phi_{n}(\omega, F)=\tilde{\phi}_{n}^{*}(\omega, F), \quad \phi_{n}(\omega, F)=\tilde{\phi}_{-n}^{*}(-\omega, F) .
$$

Since the phases of the coefficients $\phi_{0}$ and $\tilde{\phi}_{0}^{*}$ are not important, we can set $\tilde{\phi}_{0}^{*}=\phi_{0}$, and thus $\tilde{\phi}_{0}^{*} \phi_{0}=\phi_{0}{ }^{2}$. Note that the normalization factor for the QQES has an especially simple and transparent form for the case of circular polarization $(l=0)$, when only the coefficient $\phi_{0}=f_{0}$ is nonzero:

$$
\left.4 \pi \phi_{0}^{2} \frac{\partial}{\partial \mathcal{E}}\left[\sqrt{\mathcal{E}}-M_{\text {circ }}(\mathcal{E})\right]\right|_{\mathcal{E}=E}=1 .
$$

$M_{\text {circ }}(E)$ is the matrix element $M_{0,0}(E)$ with $l=0$, which enters a transcendental equation for the quasienergy in this case [131] (see also the discussion of (B.6) below):

$$
\sqrt{E}-M_{\text {circ }}(E)=1 \text {. }
$$

\section{Appendix B. Perturbative and strong field results for the coefficients $\phi_{n}$ and $f_{n}$}

The formal procedures for the correct numerical evaluation of the complex quasienergy and the Fourier coefficients of the QQES wavefunction at the origin $(r \rightarrow 0)$ are described in section 2.3. An exact numerical solution of the eigenvalue problem for $\epsilon$ and $\phi_{n}$ in (28) and (27) is a formidable task. Below, in sections B.3 and B.4 we present some details of such calculations and examples of exact numerical results for the coefficients $\phi_{n}$ and $f_{n}$ as well as analytical approximations for $f_{n}$ in the strong field regime. Simple analytical expressions may be derived in the PT approach (including higher-order corrections to the LOPT result). We present below these perturbative expansions in two forms: 
(i) in terms of the BW PT expansion, which involves the exact quasienergy $\epsilon$, i.e. assuming the complex parameter $E=u_{p}-\epsilon$ is exactly known;

(ii) in terms of the standard RS PT expansion, which involves the real, zero-order quasienergy $\epsilon_{0}=E_{0}$, i.e. $E=-E_{0}=1$. Of course, the BW results are more accurate than the RS results, especially for frequencies near thresholds. Using the PT expansion for $\epsilon$, the BW results may be easily transformed to the standard RS form.

\section{B.1. Brillouin-Wigner expansions}

To obtain the power expansion (in $F^{2}$ ) for the coefficients $\phi_{n}$ in (27), we use the expansion of the matrix elements $M_{0 k}(E)$ in (29) in an absolutely convergent series in $u_{p}$ [127]. This expansion may be written as

$M_{0 k}(E)=\frac{(-l)^{k}}{2^{k} k !} \sum_{n=\delta_{k, 0}}^{\infty} \frac{1}{n !}\left(\frac{F^{2}}{\omega^{4}}\right)^{(n+k)}{ }_{2} F_{1}\left(-\frac{n}{2}, \frac{1-n}{2} ; k+1 ; l^{2}\right) \mathcal{D}_{n+k}(E-k \omega)$,

where ${ }_{2} F_{1}$ is the hypergeometric polynomial of $l^{2}$ having the same order, $m$, for both $n=2 m$ and $n=2 m+1$. The important function $\mathcal{D}$ in (B.1) is defined by

$$
\mathcal{D}_{n}(E-p \omega)=\frac{(2 n) ! !}{2 n+1} \sum_{m=-n}^{n}(-1)^{(m+1)} \frac{(E-p \omega+m \omega)^{n+\frac{1}{2}}}{(n+m) !(n-m) !} .
$$

In accordance with the boundary conditions for the QQES wavefunctions, the square root, $\sqrt{E-k \omega}$, is defined as

$$
\begin{array}{ll}
\operatorname{Re} \sqrt{E-k \omega}>0, & \text { for } k<\operatorname{Re} E / \omega, \\
\operatorname{Im} \sqrt{E-k \omega}<0, & \text { for } k>\operatorname{Re} E / \omega .
\end{array}
$$

Note that for small $\omega$ the function $\mathcal{D}_{n}(E-p \omega)$ vanishes as $\sim \omega^{2 n}$ :

$$
\lim _{\omega \rightarrow 0} \mathcal{D}_{n}(E-p \omega) \simeq(|2 n-3|) ! ! \sqrt{E}\left(\frac{\omega^{2}}{4 E}\right)^{n}
$$

We present below results for $M_{0, k}(E)$ up to terms of orders $F^{2}$ and $F^{4}$. Only the following matrix elements are nonzero for this case:

$$
\begin{aligned}
& M_{0,0}(E)=\frac{F^{2}}{\omega^{4}}\left[\mathcal{D}_{1}(E)+\frac{F^{2}}{2 \omega^{4}}\left(1+\frac{l^{2}}{2}\right) \mathcal{D}_{2}(E)\right], \\
& M_{0,1}(E)=-\frac{l}{2} \frac{F^{2}}{\omega^{4}}\left[\mathcal{D}_{1}(E-\omega)+\frac{F^{2}}{\omega^{4}} \mathcal{D}_{2}(E-\omega)\right], \\
& M_{0,2}(E)=\frac{l^{2} F^{4}}{8 \omega^{8}} \mathcal{D}_{2}(E-2 \omega) .
\end{aligned}
$$

The above equations allow us obtain the 'BW' results for the coefficients $\phi_{ \pm k}$ with an accuracy up to $\sim F^{4}$ :

$$
\begin{aligned}
& \phi_{1}=-\frac{l}{2} \frac{F^{2}}{\omega^{4}} \frac{\phi_{0}}{\sqrt{E-2 \omega}-1}\left[\mathcal{D}_{1}(E-\omega)+\frac{F^{2}}{\omega^{4}}\left(\mathcal{D}_{2}(E-\omega)+\frac{\mathcal{D}_{1}(E-\omega) \mathcal{D}_{1}(E-2 \omega)}{\sqrt{E-2 \omega}-1}\right)\right], \\
& \phi_{2}=\frac{l^{2}}{8}\left(\frac{F^{2}}{\omega^{4}}\right)^{2} \frac{\phi_{0}}{\sqrt{E-4 \omega}-1}\left[\mathcal{D}_{2}(E-2 \omega)+2 \frac{\mathcal{D}_{1}(E-3 \omega) \mathcal{D}_{1}(E-\omega)}{\sqrt{E-2 \omega}-1}\right] .
\end{aligned}
$$

The coefficients $\phi_{n}$ with negative indices are given by $\phi_{-n}(\omega)=\phi_{n}(-\omega)$. 
The result for the normalization factor $\phi_{0}^{2}$ up to terms $\sim F^{4}$ and involving the exact $E$ can be obtained from (A.3), taking into account the above BW expansions for $M_{0, k}(E)$ and $\phi_{n}$ :

$$
\phi_{0}^{2}=\left[4 \pi \frac{\partial F(E, \omega, F)}{\partial E}\right]^{-1},
$$

where the result for $F(E, \omega, F)$ is

$$
\begin{aligned}
F(E, \omega, F)= & \sqrt{E}-\frac{F^{2}}{\omega^{4}} \mathcal{D}_{1}(E) \\
& -\frac{1}{4}\left(\frac{F^{2}}{\omega^{4}}\right)^{2}\left[\left(2+l^{2}\right) \mathcal{D}_{2}(E)+l^{2}\left\{\frac{\mathcal{D}_{1}^{2}(E+\omega)}{\sqrt{E+2 \omega}-1}+\frac{\mathcal{D}_{1}^{2}(E-\omega)}{\sqrt{E-2 \omega}-1}\right\}\right] .
\end{aligned}
$$

Taking into account only the term $\sim F^{2}$, one obtains for $\phi_{0}^{2}$

$$
\phi_{0}^{2}=\frac{\sqrt{E}}{2 \pi}\left[1+\frac{F^{2}}{\omega^{4}}(\sqrt{E(E+\omega)}+\sqrt{E(E-\omega)}-2 E)\right] .
$$

It is an interesting fact that the equation,

$$
F(E, \omega, F)=1,
$$

coincides with the transcendental equation for $\epsilon$ (or for $E$ ), which follows from the expansion (31), taking into account only terms up to $\sim F^{4}$ on the rhs of (31). Straightforward calculations show that the results in (B.6) and (B.9) for a general elliptic polarization are valid to an arbitrary order $F^{2 n}$ (taking into account, of course, terms $\sim F^{2 n}$ in the expression for $F(E, \omega, F)$ ), which is equivalent to the result in (A.5) and (A.6) for the case of circular polarization. Generally speaking, calculating the parameter $E=E(F)$ as the (nonperturbative) solution of the transcendental equation (B.9) with the boundary condition $E \rightarrow 1$ as $F \rightarrow 0$, we obtain a complicated, non-polynomial dependence of the BW expansions for $\phi_{n}$ and $\phi_{0}^{2}$ on the field amplitude $F$. Thus these results are much more accurate than standard (polynomial in $F$ ) RS expansions.

\section{B.2. Rayleigh-Schrödinger results}

In the LOPT, the coefficients $\phi_{ \pm k}$ with $k>0$ are proportional to $F^{2 k}$ and $\phi_{0} \simeq N=1 / \sqrt{2 \pi}$ (see (9)). The perturbative solution of equation (27) in the LOPT gives a simple result for $\phi_{ \pm k}$ for any $k$ :

$$
\phi_{ \pm k}=\frac{1}{\sqrt{2 \pi}}\left(\frac{l F^{2}}{\omega^{4}}\right)^{k} \chi_{k}( \pm \omega)
$$

where $\chi_{k}(\omega)$ is determined by the recurrence relation

$$
\chi_{k}(\omega)=-\frac{\mathrm{i}}{1+\mathrm{i} \sqrt{2 k \omega-1}} \sum_{j=1}^{k} \varphi_{j, k}(\omega) \chi_{k-j}(\omega),
$$

with the initial value $\chi_{0}=1$. The function $\varphi_{j, k}(\omega)$ in (B.11) is given by

$$
\varphi_{j, k}(\omega)=\frac{1}{(2 j+1) !} \sum_{m=0}^{2 j}(-1)^{m+j} C_{2 j}^{m}[(2 k-m) \omega-1]^{j+\frac{1}{2}},
$$

where $C_{n}^{m}$ is a binomial coefficient. $\varphi_{j, k}$ is simply related to the function $\mathcal{D}$ in (B.2):

$$
\varphi_{j, k}(\omega)=\mathrm{i} \frac{(-1)^{j}}{(2 j) ! !} \mathcal{D}_{j}(1-(2 k-j) \omega) .
$$


To obtain higher-order corrections to the LOPT result (B.10), the RS expansion of the quasienergy $\epsilon$ is necessary. Taking into account only the corrections $\sim F^{2}$ and $F^{4}, \epsilon$ may be written in terms of the (polarization-independent) dynamic polarizability, $\alpha(\omega)$, and the hyperpolarizability, $\gamma_{4}(\omega ; l)$, which depends on both the frequency and the polarization state of a laser field:

$$
\epsilon=-1-\frac{\alpha(\omega)}{4} F^{2}-\frac{\gamma_{4}(\omega ; l)}{24} F^{4}
$$

where the iterative in $F$ solution of equation (B.9) using $F(E, \omega, F)$ in (B.7) gives expressions for the polarizability and hyperpolarizability in terms of $\mathcal{D}_{1}(1)$ and $\mathcal{D}_{2}(1)$ functions. Taking into account the explicit form of these functions, final results coincide with those obtained previously in [81] by direct perturbative calculations (see also [188] on an alternative way to calculate $\alpha(\omega))$ :

$$
\begin{aligned}
& \alpha(\omega)=-\frac{2}{\omega^{2}}+\frac{8}{3 \omega^{4}}\left[(\omega+1)^{3 / 2}-2+\mathrm{i}(\omega-1)^{3 / 2}\right], \\
& \gamma_{4}(\omega ; l)=\frac{8}{15 \omega^{8}}\left[\left(f_{\xi}(\omega)+f_{\xi}(-\omega)\right) \xi^{2}+\left(f_{l}(\omega)+f_{l}(-\omega)\right) l^{2}\right],
\end{aligned}
$$

where the functions $f_{\xi}(\omega)$ and $f_{l}(\omega)$ are defined by

$$
\begin{aligned}
f_{\xi}(\omega)=45 \omega^{2} & +96+5 i\left(\omega^{2}-1\right)^{1 / 2}\left(\omega^{2}-7\right) \\
& -4 i(\omega-1)^{1 / 2}\left(3 \omega^{2}+14 \omega-32\right)+3 i(2 \omega-1)^{5 / 2}, \\
f_{l}(\omega)=60 \omega^{2}+ & 124+5 \mathrm{i} \sqrt{\omega^{2}-1}\left(\omega^{2}-7\right)-\frac{1}{\omega}\left[10(\omega-1)^{5 / 2}(2 \omega-1)^{1 / 2}-\mathrm{i}(2 \omega-1)^{1 / 2}\right. \\
& \left.\times\left(3 \omega^{3}+12 \omega^{2}-23 \omega+10\right)-2 \mathrm{i}(\omega-1)^{1 / 2}\left(\omega^{3}-42 \omega^{2}+76 \omega-5\right)\right] .
\end{aligned}
$$

Note that we define $\sqrt{-a}$ as $\mathrm{i} \sqrt{a}$ for $a>0$. The hyperpolarizability $\gamma_{4}(\omega ; l)$ is an important atomic parameter and involves a complicated combination of fourth-order PT matrix elements (see, e.g., [141]). For $\mathrm{H}^{-}, \gamma_{4}(\omega ; l)$ (for the case of linear polarization) was calculated, taking into account electron correlations, perturbatively (in $F$ ) in [189] (in the static limit $\omega=0$ ) as well as nonperturbatively (in $F$ ) $[108,190]$. It is somewhat surprising that the simple $\delta$-model result in (B.16)-(B.18) is in close agreement with results of these sophisticated calculations (see comparisons in [191], where the $\delta$-model result for the dynamical hyperpolarizability of $\mathrm{H}^{-}$in the presence of a strong static electric field is also presented).

To calculate the $F$-dependent RS corrections to the normalization parameter, $\phi_{0}=$ $1 / \sqrt{2 \pi}+\phi_{0}^{(1)}+\cdots$, the relations (B.6) and (B.7) may be used, taking into account the expansion (B.14) for the quasienergy. One obtains for the lowest order in $F$ correction:

$\phi_{0}{ }^{(1)}=\frac{1}{\sqrt{2 \pi}} \frac{F^{2}}{2 \omega^{4}}\left\{\sqrt{\omega+1}-2-\mathrm{i} \sqrt{\omega-1}+\frac{1}{3}\left[(\omega+1)^{3 / 2}-2+\mathrm{i}(\omega-1)^{3 / 2}\right]\right\}$.

Note that the normalization factor for the QQES solution is unconventional because it is complex; see (A.3) or (B.6) (in LOPT the complexity appears only for above-threshold frequencies, $\omega>1$ ). Nevertheless, this complexity is not surprising in the theory of quasistationary states for radiationless problems (see, e.g., [192]); it originates from an analytic continuation of the standard normalization convention to the case of the QQES.

B.2.1. Analytical properties of $\phi_{ \pm k}(\omega)$ and threshold phenomena. We discuss now in more detail the analytical structures of the coefficients $\phi_{ \pm k}$, which are essential for the analysis of ADs. For clarity, we present here an explicit RS form of the coefficients $\phi_{1}$ and $\phi_{2}$, which enter the LOPT result for the ADs for $n \leqslant 5$ :

$$
\phi_{1}=\frac{l F^{2}}{\sqrt{2 \pi} 6 \omega^{4}} g_{1}(\omega), \quad \phi_{2}=\frac{l^{2} F^{4}}{\sqrt{2 \pi} 36 \omega^{8}} g_{2}(\omega),
$$


where

$$
\begin{aligned}
g_{1}(\omega) \equiv 3 \chi_{1}(\omega) & =\frac{\mathrm{i}(2 \omega-1)^{3 / 2}-2 \mathrm{i}(\omega-1)^{3 / 2}+1}{1+\mathrm{i} \sqrt{2 \omega-1}} \\
g_{2}(\omega) \equiv 9 \chi_{2}(\omega) & =\mathrm{i} g_{1}(\omega)\left[\frac{(4 \omega-1)^{3 / 2}-2(3 \omega-1)^{3 / 2}+(2 \omega-1)^{3 / 2}}{1+\mathrm{i} \sqrt{4 \omega-1}}\right] \\
- & \frac{3}{10} \frac{\mathrm{i}(4 \omega-1)^{5 / 2}-4 \mathrm{i}(3 \omega-1)^{5 / 2}+6 \mathrm{i}(2 \omega-1)^{5 / 2}-4 \mathrm{i}(\omega-1)^{5 / 2}-1}{1+\mathrm{i} \sqrt{4 \omega-1}} .
\end{aligned}
$$

Most important is the complexity of $\phi_{k}$, whose imaginary part, $\operatorname{Im} \phi_{k}$, is different in each frequency interval $1 / p<\omega<1 /(p-1)$, for $p=1,2, \ldots$. This fact becomes rather clear by introducing the 'phase function', $\delta_{0}(E)$, of $E$ (which is in general complex) as follows:

$$
\mathrm{e}^{\mathrm{i} \delta_{0}(E)}=\frac{1-\mathrm{i} \sqrt{E}}{\sqrt{1+E}} .
$$

For positive $E, \delta_{0}(E)$ is the well-known phase shift of the continuum S-state for a shortrange potential. (Other scattering phases, $\delta_{L}(E)$, are zero in the $\delta$-model potential.) Thus $\mathrm{e}^{\mathrm{i} \delta_{0}(E)}=\cos \delta_{0}(E)+\mathrm{i} \sin \delta_{0}(E)$. On the contrary, for negative arguments, $E=-E^{\prime}$, in the interval $-1<E \leqslant 0$ the exponential (B.21) is real:

$$
\mathrm{e}^{ \pm \mathrm{i} \delta_{0}\left(-E^{\prime}\right)}=\frac{1 \mp \sqrt{E^{\prime}}}{\sqrt{1-E^{\prime}}} .
$$

Thus, equation (B.21) gives an analytical continuation of the scattering phase to the region of negative $E$. Note that at $E=E_{0}=-1$ we obtain the well-known result, $\cot \delta_{0}\left(E_{0}\right)=i$, which determines the poles of the $S$ matrix at the bound state energies. A detailed analysis shows that $\phi_{ \pm k}$ in any PT order may be presented as a combination of exponentials (having the form (B.21), with different $E=p \omega \mp 1$, where $p$ is an integer) and regular functions of $\omega$ (having the form $(m \omega \mp 1)^{n}$, where $m$ and $n$ are integers). For instance, the functions $g_{1}$ and $g_{2}$ in (B.20) may be re-written in terms of $\delta_{0}$ as follows:

$$
\begin{aligned}
g_{1}(\omega)=2 \omega- & 1-\sqrt{2}(\omega-1) \mathrm{e}^{\mathrm{i}\left[\delta_{0}(2 \omega-1)-\delta_{0}(\omega-1)\right]}, \\
g_{2}(\omega)=g_{1}(\omega) & {\left[4 \omega-1-\mathrm{e}^{\mathrm{i} \delta_{0}(4 \omega-1)}\left(\sqrt{3}(3 \omega-1) \mathrm{e}^{-\mathrm{i} \delta_{0}(3 \omega-1)}+\frac{1}{\sqrt{2}}(2 \omega-1) \mathrm{e}^{-\mathrm{i} \delta_{0}(2 \omega-1)}\right)\right] } \\
+ & \frac{3}{5} \mathrm{e}^{\mathrm{i} \delta_{0}(4 \omega-1)}\left[\sqrt{3}(3 \omega-1)^{2} \mathrm{e}^{-\mathrm{i} \delta_{0}(3 \omega-1)}-\frac{3}{\sqrt{2}}(2 \omega-1)^{2} \mathrm{e}^{-\mathrm{i} \delta_{0}(2 \omega-1)}\right. \\
+ & \left.(\omega-1)^{2} \mathrm{e}^{-\mathrm{i} \delta_{0}(\omega-1)}\right]-\frac{3}{10}(4 \omega-1)^{2} .
\end{aligned}
$$

These equations (together with (B.20)) demonstrate that $\phi_{k}$ is complex entirely because of the scattering phases: a new phase (say, $\delta_{0}(2 m \omega-1)$ with $m<k$ ) contributes to $\phi_{k}$ when the frequency $\omega$ increases from $\omega<1 / 2 m$ to $\omega>1 / 2 m$ and, thus, a new detachment channel (with the absorption of two additional photons) is opened. For instance, the function $g_{1}(\omega)$ is real for $\omega<1 / 2$; for $1 / 2<\omega<1, g_{1}(\omega)$ is complex because of the phase $\delta_{0}(2 \omega-1)$; and for $\omega>1$ an additional contribution to the imaginary part of $g_{1}$ appears because of the phase $\delta_{0}(\omega-1)$. Similarly, $g_{2}(\omega)$ is real only for $\omega<1 / 4$. Therefore, the entire set of coefficients $\phi_{ \pm k}$ is real if the phases $\phi_{k}$ are neglected, i.e. if the interactions of the escaping electron with the parent atom in the final state as well as in intermediate states (i.e. after absorption of $m<k$ photons) are neglected. Note that the phase dependence (and the complexity) of the coefficients $\phi_{k}$ may be established also by direct PT calculations of Fourier harmonics of the S-wave part of the QQES wavefunction $\Phi_{\epsilon}(r, t)$ (which is given at $r \rightarrow 0$ in terms of the coefficients 
$\left.\phi_{ \pm k}\right)$, using the known Green function for an electron in a $\delta$-model potential. However, such a calculation is much more tedious than the PT expansions of the exact results presented in section 2 .

The general features of the frequency dependence of the $\phi_{k}$ coefficients discussed above provide an instructive illustration of the so-called threshold phenomena that are predicted by the general theory of multichannel reactions involving a short-range binding potential (see, e.g., [186]). For this kind of potential, in a classic paper [70] Wigner showed that the branchpoint singularity of the reaction amplitude at the threshold energy $(E=0)$ of a given breakup channel having an orbital angular momentum $L$ causes a universal threshold behaviour of the corresponding breakup cross section:

$$
\sigma_{L} \propto E^{L+1 / 2},
$$

above the opening of this channel. Extending Wigner's analysis, Baz' [187] showed that generally in a multichannel problem the branch-point non-analyticity of the multichannel amplitude at the $m$ th-channel threshold, $E_{m}=0$, causes a particular, non-analytic behaviour, or TA, in the partial cross sections of all other open channels, in general. These TAs are well known and described in textbooks (see, e.g., [148] and section 147 in [142]). Laser detachment of a negative ion provides a good example of a multichannel problem where TA effects can have a great influence on partial cross sections at the opening or closure of a multiphoton detachment channel owing to a variation of either the laser frequency or intensity. (In the latter case, the closure originates from ponderomotive shift effects.) In the QQES problem for a zero-range potential (in which case only the S-wave scattering phases $\delta_{0}(E)$ provide the interchannel couplings), all the non-analyticities are concentrated exclusively in the $S$-wave part of the QQES solution, i.e. in the coefficients $\phi_{k}$, as is obvious from equations (21) and (23). The threshold branch-point singularities of $\phi_{k}$ are evident from the explicit form (B.1) for the matrix elements $M_{0 k}$, which enter the exact equation (27) satisfied by $\phi_{k}$. Of course, for an arbitrary $F$, the position of the branch point for the $m$ th threshold:

$$
m \omega-E \equiv m \omega+\epsilon-u_{p}=0,
$$

is shifted to the complex plane of $F$ and $\omega$. However, in the RS PT approach, the TAs occur at real frequencies, $m \omega-1=0$, and cause the specific frequency dependence of the $\phi_{k}$ coefficients discussed above.

\section{B.3. Exact strong field results}

In the strongly nonperturbative regime, the use of the expansion (B.1) for numerical calculations of the matrix elements $M_{0 k}(E)$ becomes ineffective because of the slow convergence of the series in $\left(F / \omega^{2}\right)^{2}$ for $\omega<1$. Moreover, as discussed below, for strong $F$ the direct numerical calculation of the coefficients $f_{n}$ using the matrix elements $\tilde{M}_{n n^{\prime}}(E)$ is preferable to use of the relation (24) for their calculation in terms of $\phi_{n}$. Note that the integral (29) (as well as the similar integral for $\tilde{M}_{n n^{\prime}}(E)$ ) is formally divergent for $\tau \rightarrow \infty$ because of the negative imaginary part of the quasienergy $\epsilon$ (see the above discussion of equation (13)). The numerical calculation of such integrals is possible by using the following analytic continuation procedure [134]:

$$
\begin{aligned}
\int_{0}^{\infty} \frac{\mathrm{d} \tau}{\tau^{1 / 2}} \mathrm{e}^{-\mathrm{i} \alpha \tau} f(\tau) & =\int_{0}^{\infty} \frac{\mathrm{d} \tau}{\tau^{1 / 2}} \mathrm{e}^{-\mathrm{i} \alpha \tau} \int_{-\infty}^{\infty} \mathrm{d} \tau^{\prime} \delta\left(\tau^{\prime}-\tau\right) f\left(\tau^{\prime}\right) \\
& =\int_{-\infty}^{\infty} \mathrm{d} \tau^{\prime} \int_{0}^{\infty} \frac{\mathrm{d} \tau}{\tau^{1 / 2}} \mathrm{e}^{-\mathrm{i} \alpha \tau} \int_{-\infty}^{\infty} \frac{\mathrm{d} k}{2 \pi} \mathrm{e}^{\mathrm{i} k\left(\tau^{\prime}-\tau\right)} f\left(\tau^{\prime}\right) \\
& =\frac{1}{\sqrt{4 \pi \mathrm{i}}} \int_{-\infty}^{\infty} \frac{\mathrm{d} k}{\sqrt{\alpha+k}} \int_{-\infty}^{\infty} \mathrm{d} \tau \mathrm{e}^{\mathrm{i} k \tau} f(\tau) .
\end{aligned}
$$



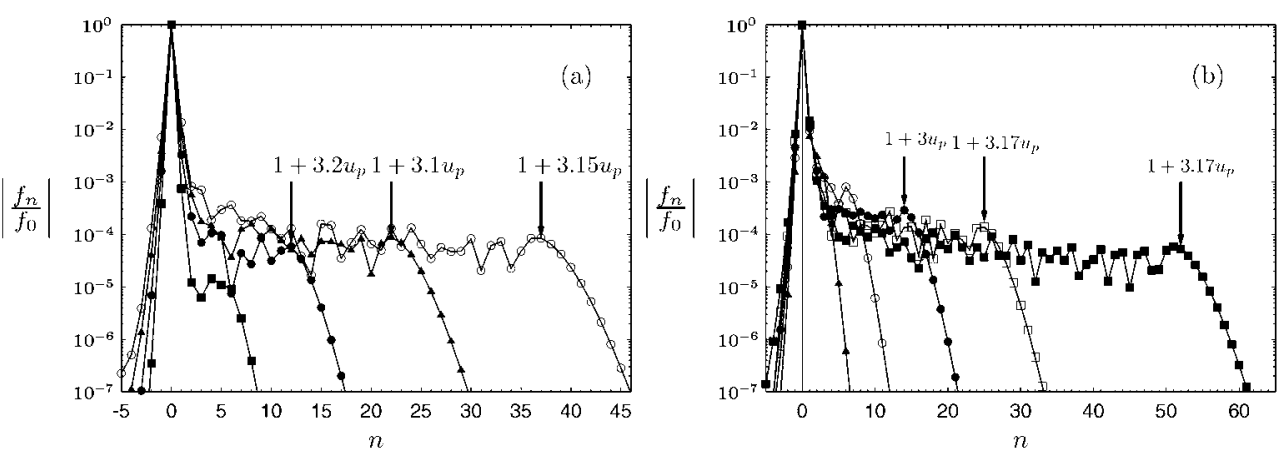

Figure B.1. $n$ dependence of the Fourier coefficients $f_{n}$ for linear laser polarization. (a) Exact values of $\left|f_{n} / f_{0}\right|$ for different $F$ and $\omega=0.155$. Full squares: $F=0.1$; full circles: $F=0.2$; triangles: $F=0.3$; empty circles: $F=0.4$. Arrows mark the cut-offs in units of the energy (see (B.29)). Full curves serve to guide the eye. (b) Exact values of $\left|f_{n} / f_{0}\right|$ for different $\omega$ and $F=0.4$. Full squares: $\omega=0.138$; empty squares: $\omega=0.178$; full circles: $\omega=0.218$; empty circles: $\omega=0.298$; full triangles: $\omega=0.458$.

The above formula involves a Fourier transform (that can be calculated very efficiently using a Fast Fourier Transform algorithm) followed by a second integration, which can also be performed numerically with high accuracy. The finite system of equations, obtained from (27) (or from a similar system for $f_{n}$ ) after truncating the ranges of $n$ and $n^{\prime}$ to an appropriate finite number of values, can then be solved numerically. One obtains thereby the eigenvalues $\epsilon$ as well as the Fourier coefficients $\phi_{n}$ ( or $f_{n}$ ) of the QQES state at the origin. Note that Mur et al [193] suggest using the Zel'dovich regularization for singular integrals like the first one in equation (B.27), i.e. introducing a regularization factor $\exp \left(-\sigma \tau^{2}\right)$ with $\sigma \rightarrow+0$ [148, 194]. They demonstrate a reasonable convergence (with decreasing $\sigma$ ) of this method in calculations of $\epsilon$ for a circularly polarized field (where only one matrix element, $M_{00}$, is necessary). However, the applicability of this regularization method for the cases of linear or elliptic polarization is questionable since in these cases a large number of matrix elements $M_{n n^{\prime}}$ (29) calculated with high precision are necessary for the solution of the determinantal equation (28). In addition, as shown in [193], the proposed method of regularization in [193] is inapplicable for calculations of 'near-threshold' matrix elements, when $\operatorname{Im} \epsilon$ is of the order of (or larger than) $|\operatorname{Re} \epsilon+k \omega|$.

In figure B.1 we present the dependence of the exactly calculated coefficients $f_{n}$ as functions of $F$ (in figure B.1(a)) and $\omega$ (in figure B.1(b)) in the nonperturbative regime. Recall that, in the KA limit, $f_{n}^{K A}=\delta_{n 0} / \sqrt{2 \pi}$. As we have noted after equation (B.19), in general the normalization factor of a QQES wavefunction (i.e. $\phi_{0}$ or $f_{0}$ ) is complex. However, the magnitude of the imaginary part of $f_{0}$ is very small for the values $F$ and $\omega$ considered in figure B.1 and, actually, it has the same magnitude as the imaginary part of the quasienergy: $\sim\left(10^{-4}-10^{-3}\right)$. Moreover, the modulus of the normalization factor $f_{0}$ for this case is close to the zero-field value $1 / \sqrt{2 \pi}$, decreasing only by about $2 \%$ at $F \approx 0.5$. With increasing $F$, the negative- $n$ and a few of the lowest positive- $n$ coefficients exhibit typical (smoothly decreasing) perturbative behaviour. However, even for weak intensities (e.g. for $I=F^{2}=10^{-2}$ in figure B.1(a)), the plateau signature characterizing the dependence of $f_{n}$ on $n$ appears starting at some $n \approx n_{t h}$, and a well-developed plateau structure is formed in the strongly nonperturbative regime, $\gamma<1$, having a cut-off at some $n \approx n_{c}$.

To explain the behaviour of the coefficients $f_{n}$ described above and to estimate $n_{t h}$ and $n_{c}$, we emphasize that the coefficients $f_{n \neq 0}$ vanish in the KA limit and that these coefficients 
originate from the simultaneous interaction of the electron with both the laser field and the binding potential. Concerning the onset of the plateau, we note that (in weak fields) the PT result for $f_{n}$ may be presented as: $f_{n}=c_{n}(\omega) F^{2|n|}$ (cf the similar results (B.10)-(B.12) for the $\phi_{n}$ coefficients). Thus, for fixed $F$ and $\omega$, the breakdown of a smooth decrease of $f_{n}$ with increasing $n, f_{n} \sim F^{2|n|}$, originates only from the appearance of an irregularity (nonanalyticity) in the behaviour of the factor $c_{n}(\omega)$ starting from $n=n_{t h}$. One may observe the effects of this singularity in figure B.1(a), where the $F=0.1$ curve shows a sudden rise above $n=3$ and where the $F=0.2$ curve shows a similar rise, again above $n=3$. Note that, with increasing $F$, the location of these singularities does not necessarily occur at integer values of $n$, so that not every curve in figure B.1(a) shows the singularity clearly. Nevertheless, the calculations with smaller steps in $F$ (as in figure B.1(a)) show that the position of $n_{t h}$ is largely intensity-independent up to high values of $F$, i.e. it has a PT origin. In the PT limit, the value of $n_{t h}$ may be estimated owing to the analytical structure of the perturbative results for the coefficients $f_{n}$ that involve characteristic branch-point terms $\sqrt{p \omega+1}$ with $p= \pm 1, \ldots, \pm 2 n$ (see (B.10)-(B.12) and the explicit expressions (B.20) for the $\phi_{n}$ coefficients having $n= \pm 1$ and \pm 2 ). It is clear that, at fixed $\omega$, the first irregularity in the dependence of $f_{n}$ on $n$ with increasing $n$ should appear for $n=n_{t h}$, which is defined by $\left(1-2 n_{t h} \omega\right) \leqslant 0$, i.e. $2 n_{t h}$ is simply the lowest even (open) $U_{p}$-unshifted photodetachment channel for a given value of $\omega$. Thus, one obtains the following estimate for $n_{t h}$ :

$$
2 n_{t h} \omega \approx 1 \quad \text { (or } E_{0}+2 n_{t h} \hbar \omega \geqslant 0 \text { in absolute units). }
$$

This estimate is in good agreement with numerical values of $n_{t h}$, both for different $F$ (in figure B.1(a)) and $\omega$ (in figure B.1(b)) and shows that in a rigorous treatment the onset of the plateau feature in the spectrum of $f_{n}$ originates from purely quantum (zero-field threshold) effects. In contrast, the position of the cut-offs in the spectra for $f_{n}$ in figure B.1 may be explained using classical considerations in terms of rescattering effects. Indeed, the coefficients $f_{n}$ are proportional to the amplitudes for the electron (having the energy $E_{N}=\operatorname{Re} \epsilon+2 n \omega$ ) at the origin (see (20) and (23)). The number $N=2 n$ is even since the probability density at the origin is determined by the $S$-wave component of $\Phi_{\epsilon}(r, t)$, i.e. in view of electric dipole selection rules, the electron can return to the parent atom only after absorption or emission of an even number of photons, when the S-wave component of angular momentum is nonzero (see the discussion above equation (23)). Figure B.1 shows that only electrons with positive energies up to $E_{2 n_{c}}=\operatorname{Re} \epsilon+2 n_{c} \omega$ have a significant probability density to be at the origin. To estimate $n_{c}$, it is reasonable to equate $E_{2 n_{c}}$ with the maximum energy of the 'rescattered' electrons at the origin, which is well known from numerical quantum calculations [195] as well as from a simple classical analysis [132]: $E_{c} \approx 3.17 U_{p}$. Thus, we obtain the following estimation for $n_{c}$ :

$$
2 n_{c} \omega \approx 1+3.17 u_{p},
$$

which agrees very well with our numerical results for $n_{c}$ in figure B.1 for strong $F$ and/or small $\omega$, i.e. for small values of the Keldysh parameter $\gamma$.

The behaviour of the $\phi_{n}$ coefficients is significantly different from that for $f_{n}$. Numerical evaluations demonstrate that, with increasing $F$, the coefficients $\phi_{n}$ undergo large variations and grow rapidly with increasing $|n|$ for both $n>0$ and $n<0$. For example, we present in figure B.2 the $F$ dependence of the normalization constant $\phi_{0}$ and the coefficients $\phi_{n}$ for the particular frequency $\omega=0.155$ and for linear laser polarization. For circular polarization, $\phi_{0}^{\text {circ }}$ coincides with $f_{0}^{c i r c}$ and differs only slightly from $1 / \sqrt{2 \pi}$. In contrast, for linear polarization we have a large number of contributing coefficients $\phi_{n}$, which vanish at $F=0$ but which have increasing contributions as the intensity increases. Therefore, $\sqrt{2 \pi}\left|\phi_{0}\right|$ needs to be decreasing 

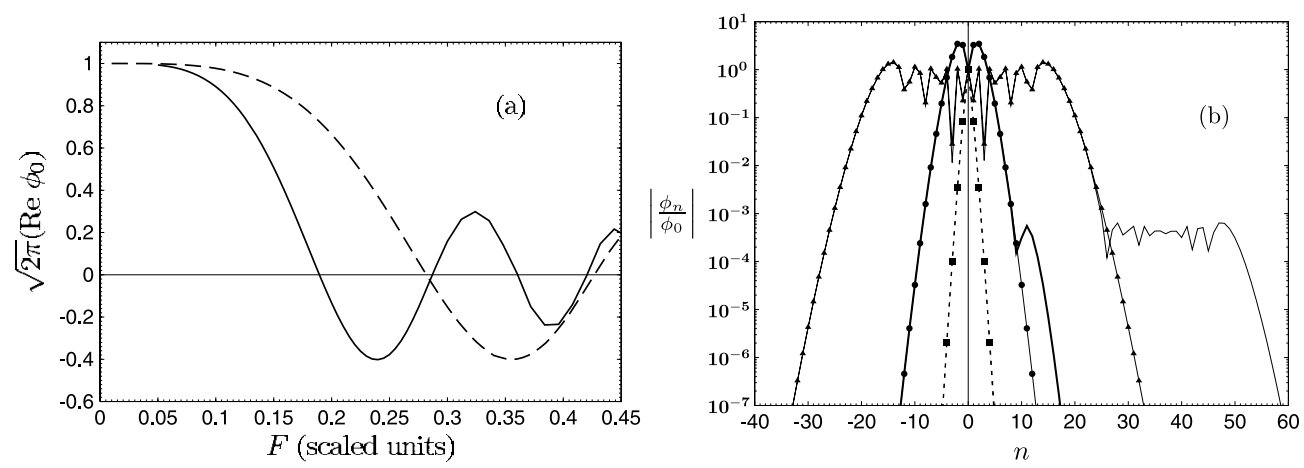

Figure B.2. (a) $F$ dependence of the normalization factor $\phi_{0}$ for the case of linear laser polarization ( $l=1$ ) at $\omega=0.155$ (full curve) and $\omega=0.202$ (broken curve). (b) Dependence of the ratio, $\left|\phi_{n} / \phi_{0}\right|$, on $n$ (at $\omega=0.155$ and $l=1$ ) for three values of the laser amplitude: $F=0.05$ (dotted curve); $F=0.20$ (thick full curve) $F=0.49$ (thin full curve). Squares, circles and triangles are results of the KA (equation (44)) for $F=0.05,0.20$ and 0.49 , respectively.

in order to maintain the unit normalization of $\Phi_{\epsilon}(r, t)$. Although the imaginary part of $\phi_{0}$ is very small, $\sim\left(10^{-4}-10^{-3}\right)$, its real part reveals big variations. It is interesting that the exact results for $\sqrt{2 \pi} \operatorname{Re} \phi_{0}$ in figure B.2(a) are in excellent agreement with those of the KA (44): the difference between the exact and the KA results is less than $1 \%$.

For coefficients $\phi_{n}$ with $n \neq 0$, the coincidence of the exact and the KA results takes place only in the weak field domain, $\gamma>1$ (see figure B.2(b) where $\gamma=3.1$ for $F=0.05$ ). At $\gamma \sim 1(\gamma=0.775$ for $F=0.2$ in figure B.2(b)) the exact coefficients for positive $n$ start to increase above those of the KA beginning from some $n$. For high enough $F$, a twoplateau structure for $\left|\phi_{n} / \phi_{0}\right|$ for positive $n$ develops: the higher plateau for smaller values of $n$ represents the 'Keldysh regime'; the lower (beyond KA) plateau for larger values of $n$ is caused by binding potential (rescattering) effects. Numerical values of the cut-off number, $n_{c}$, for the second plateau are well approximated by the same estimate (B.29) as for the coefficients $f_{n}$. Since the KA reproduces $\phi_{n}$ well, except for those $n$ values in the second plateau domain, the origin of the first plateau is clearly independent of the binding potential and, therefore, can be explained in terms of the KA. The curve for $F=0.49$ in figure B.2(b) and numerical calculations for other values of $F$ and $\omega$ show that, with reasonable accuracy, the critical value of $n, n_{c r}$, at which the non-KA plateau begins can be obtained from the relation

$$
n_{c r} \omega \approx u_{p} \quad\left(\text { or } 2 n_{c r} \hbar \omega \equiv E_{c r} \approx 2 U_{p}\right. \text { in absolute units). }
$$

Mathematically, the value $n_{c r}$ corresponds to the value of the index $n$ in $\sqrt{2 \pi} \phi_{n}^{K A}=J_{n}\left(u_{p} / 2 \omega\right)$ at which the oscillatory behaviour of the Bessel function $J_{m}(x)$ with increasing $m$ changes to a decreasing behaviour (this happens for indices $m$ much larger than the argument $x$, and $m \sim 2 x$ corresponds to the transition domain). The estimate in (B.30) for $n_{c r}$ agrees with the known quasiclassical results on the size of the 'Keldysh plateau' in ATI processes [196]: neglecting rescattering by the binding potential, the 'Keldysh part' of the ATI plateau corresponds to an electron energy $E_{c r}$ of order $2 U_{p}$.

\section{B.4. 'Rescattering approximations' for the coefficients $f_{n}$}

An exact calculation of the coefficients $f_{n}$ is time-consuming since it requires the preliminary calculation of the complex quasienergy according to (28). A simple way to obtain $f_{n}$ approximately in the nonperturbative regime is the iterative solution of equation (27) with the 


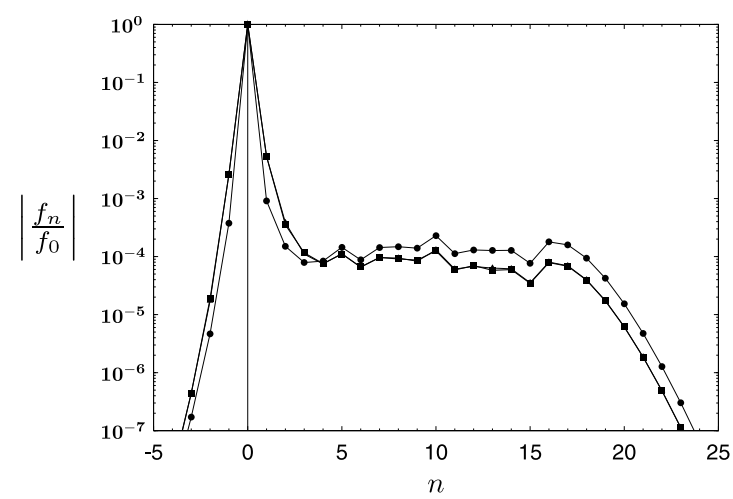

Figure B.3. Comparison of exact and approximate Fourier coefficients $f_{n}$ for the case of linear laser polarization, $\omega=0.155$, and $F=0.25$. Squares: exact results; triangles: approximation I results using (B.31); circles: approximation II results using (B.32). Note that the squares and triangles are indistinguishable on the scale of this figure.

substitution $M_{n n^{\prime}}(E) \rightarrow \tilde{M}_{n n^{\prime}}(E)$ (where $\tilde{M}_{n, n^{\prime}}(E)$ is defined in the text above (40)) starting from the KA results: $f_{n}^{K A}=\delta_{n 0} / \sqrt{2 \pi}, E=1+u_{p}$. The first iteration gives the following approximation for $f_{n \neq 0}$ :

$$
f_{n}^{(1)}=\frac{\tilde{M}_{n, 0}\left(1+u_{p}\right)}{\sqrt{1+u_{p}+2 n \omega}-1-M_{n, n}\left(1+u_{p}\right)} f_{0}^{K A} .
$$

We shall call this method 'approximation I'. Obviously, approximation I is equivalent to a perturbative (first-order) account of the binding potential (or rescattering) effects with respect to the KA results. Figure B.3 demonstrates an unexpectedly precise agreement of the approximation I coefficients $f_{n}^{(1)}$ with the exact results.

A simplified version of approximation I (denoted approximation II) consists in replacing the entire denominator of (B.31) by -1 :

$$
f_{n}^{(2)}=-\tilde{M}_{n, 0}\left(1+u_{p}\right) f_{0}^{K A}
$$

Although this approximation has no proper theoretical justification, the results of approximations I and II are in good qualitative agreement, as may be seen in figure B.3 (although, compared to approximation I, approximation II gives a less accurate estimate for the coefficients $f_{n}$, both on the plateau and beyond the plateau). Using the approximate analytical result (B.31) for $f_{n}^{(1)}$ instead of the exact numerical Fourier coefficients of $f(t-\tau)$ in (21) (see also (23)), we obtain an analytical expression for the QQES wavefunction. This may be useful in different applications that take into account the first-order binding potential corrections to the KA result (43). Similarly, the use of $f_{n}^{(1)}$ or $f_{n}^{(2)}$ in (37) gives the rescattering corrections to the KA amplitude (45). Note that the final result for the differential rate $\Gamma^{(n)}(\boldsymbol{n})$ in (38) with $\epsilon=E_{0}=-1, f_{0} \rightarrow f_{0}^{K A}$ and $f_{n \neq 0} \rightarrow f_{n}^{(2)}$ coincides with that obtained by Lohr et al [133] using a different approach for the generalization of the Keldysh amplitude to account for rescattering.

\section{Appendix C. Details of derivations for the $n$-photon detachment amplitude}

To derive the result (36) for the amplitude $\mathcal{A}_{n}$, we consider first the following singular integral:

$$
g_{\epsilon}^{(n)}(r)=\frac{\mathrm{i}}{2^{n}} \int_{0}^{\infty} \frac{\mathrm{d} \tau \exp \left[\mathrm{i} \epsilon \tau+\mathrm{i} \frac{r^{2}}{4 \tau}\right]}{(4 \pi \mathrm{i} \tau)^{3 / 2} \tau^{n}}, \quad n=0,1,2,3 \ldots
$$


For $n=0$ we have the known identity

$$
g_{\epsilon}^{(0)}(r)=\mathrm{i} \int_{0}^{\infty} \mathrm{d} \tau \frac{\exp \left[\mathrm{i} \frac{r^{2}}{4 \tau}+\mathrm{i} \epsilon \tau\right]}{(4 \pi \mathrm{i} \tau)^{3 / 2}}=\frac{\mathrm{e}^{\mathrm{i} \sqrt{\epsilon} r}}{4 \pi r},
$$

which in fact is equivalent to the Fourier transform of the time-dependent (retarded) Green function for a free electron. The identity (C.2) allows us to verify the following recurrence relation for evaluation of $g_{\epsilon}^{(n)}(r)$ for $n>0$ :

$$
g_{\epsilon}^{(n)}(r)=-\mathrm{i} \frac{\boldsymbol{r}}{r^{2}} \cdot \frac{\partial}{\partial \boldsymbol{r}} g_{\epsilon}^{(n-1)}(r), \quad n=1,2,3 \ldots
$$

The direct calculation of derivatives gives the major asymptotic term, $\sim r^{-n-1}$ :

$$
g_{\epsilon}^{(n)}(r)=\frac{\mathrm{i}}{2^{n}} \int_{0}^{\infty} \frac{\mathrm{d} \tau \exp \left[\mathrm{i} \epsilon \tau+\mathrm{i} \frac{r^{2}}{4 \tau}\right]}{(4 \pi \mathrm{i} \tau)^{3 / 2} \tau^{n}}=\frac{\mathrm{e}^{\mathrm{i} \sqrt{\epsilon} r}}{4 \pi r}\left(\frac{\sqrt{\epsilon}}{r}\right)^{n}+\cdots
$$

The Taylor expansion of the exponential factor $\exp \left(\frac{\mathrm{i}}{\omega^{2} \tau} \boldsymbol{R} \cdot \boldsymbol{F}(\omega t-\omega \tau)\right)$ in (34) leads to the following asymptotic form of $\psi_{E}(\boldsymbol{R}, t)$ at $R \rightarrow \infty$ :

$$
\begin{aligned}
\left.\psi_{E}(\boldsymbol{R}, t)\right|_{R \rightarrow \infty} & \simeq \frac{1}{\sqrt{4 \pi \mathrm{i}}} \sum_{k=-\infty}^{\infty} \sum_{p=0}^{\infty} \sum_{l=0}^{p} \phi_{k} \mathrm{e}^{\mathrm{i}(p-2 l-2 k) \omega t} \frac{(\boldsymbol{e} \cdot \boldsymbol{n})^{l}\left(\boldsymbol{e}^{*} \cdot \boldsymbol{n}\right)^{p-l}}{l !(p-l) !}\left(\frac{\mathrm{i} R F}{2 \omega^{2}}\right)^{p} \\
& \times \int_{0}^{\infty} \frac{\mathrm{d} \tau}{\tau^{p+3 / 2}} \exp \left[\mathrm{i}\left\{\frac{R^{2}}{4 \tau}+(2 k \omega+2 l \omega-p \omega-E) \tau\right\}\right],
\end{aligned}
$$

where $n=R / R$. The integral over $\tau$ is approximated by the first term on the right of (C.4). Finally, extracting from $\left.\psi_{E}(\boldsymbol{R}, t)\right|_{R \rightarrow \infty}$ the harmonic of frequency $n \omega$, we obtain (cf (35))

$$
\lim _{R \rightarrow \infty} \psi_{E}(\boldsymbol{R}, t)=\sum_{n} \mathcal{A}_{n} \frac{\mathrm{e}^{\mathrm{i} k_{n} R}}{R} \mathrm{e}^{-\mathrm{i} n \omega t}+\cdots,
$$

where $k_{n}=\sqrt{n \omega-E}$ and the $n$-photon detachment amplitude is given by

$$
\mathcal{A}_{n}=\sum_{k=-\infty}^{\infty} \sum_{p=0}^{\infty} \sum_{l=0}^{p} \phi_{k} \delta_{n, 2 l+2 k-p} \frac{(\boldsymbol{e} \cdot \boldsymbol{n})^{l}\left(\boldsymbol{e}^{*} \cdot \boldsymbol{n}\right)^{p-l}}{l !(p-l) !}\left(\frac{\mathrm{i} F}{\omega^{2}} k_{n}\right)^{p} .
$$

Taking into account the Kronecker symbol $\delta_{i, j}$ and replacing the summation index $p$ by $m$ according to $p=2 m+s$, where $s=n-2\left[\frac{n}{2}\right]$, equation (C.7) can be presented as follows:

$\mathcal{A}_{n}=\mathrm{i}^{s} \sum_{k=-\infty}^{\infty} \phi_{k} \sum_{m=m_{0}(k)}^{\infty}(-1)^{m} \frac{(\boldsymbol{e} \cdot \boldsymbol{n})^{\left[\frac{n}{2}\right]+m-k+s}\left(\boldsymbol{e}^{*} \cdot \boldsymbol{n}\right)^{m+k-\left[\frac{n}{2}\right]}}{\left(m+\left[\frac{n}{2}\right]-k+s\right) !\left(m+k-\left[\frac{n}{2}\right]\right) !}\left(\frac{F}{\omega^{2}} k_{n}\right)^{2 m+s}$,

where $m_{0}(k)=k-\left[\frac{n}{2}\right]-s$ at $k \geqslant\left(\left[\frac{n}{2}\right]+1\right)$ and $m_{0}(k)=\left[\frac{n}{2}\right]-k$ at $k \leqslant\left[\frac{n}{2}\right]$. Using the following relation:

$$
(\boldsymbol{e} \cdot \boldsymbol{n})^{m+\left[\frac{n}{2}\right]-k+s}\left(\boldsymbol{e}^{*} \cdot \boldsymbol{n}\right)^{m+k-\left[\frac{n}{2}\right]}=|\boldsymbol{e} \cdot \boldsymbol{n}|^{2 m+s}\left(\frac{\boldsymbol{e} \cdot \boldsymbol{n}}{|\boldsymbol{e} \cdot \boldsymbol{n}|}\right)^{n-2 k},
$$

and taking into account that the sum over $m$ in (C.8) gives the series representation for the Bessel function $J_{l}(x)$ with an integer index $l$ :

$$
\sum_{m=m_{0}(k)}^{\infty} \frac{(-1)^{m} z^{2 m+s}}{\left(m+\left[\frac{n}{2}\right]-k+s\right) !\left(m+k-\left[\frac{n}{2}\right]\right) !}=(-1)^{\left[\frac{n}{2}\right]-k} J_{n-2 k}(2 z),
$$

we obtain the final result (36) for $\mathcal{A}_{n}$. 


\section{References}

[1] (a) Keldysh L V 1964 Zh. Eksp. Teor. Fiz. 471945 (Engl. transl. 1965 Sov. Phys.-JETP 20 1307)

(b) Nikishov A I and Ritus V I 1966 Zh. Eksp. Teor. Fiz. 50255 (Engl. transl. 1966 Sov. Phys.-JETP 23 168)

(c) Perelomov A M, Popov V S and Terent'ev M V 1966 Zh. Eksp. Teor. Fiz. 501393 (Engl. transl. 1966 Sov. Phys.-JETP 23 924)

[2] Perelomov A M, Popov V S and Terent'ev M V 1966 Zh. Eksp. Teor. Fiz. 51309 (Engl. transl. 1967 Sov. Phys.-JETP 24 207)

Perelomov A M and Popov V S 1967 Zh. Eksp. Teor. Fiz. 52514 (Engl. transl. 1967 Sov. Phys.-JETP 25 336) Popov V S, Kuznetsov V P and Perelomov A M 1967 Zh. Eksp. Teor. Fiz. 53331 (Engl. transl. 1968 Sov. Phys.-JETP 26 222)

[3] Zon B A, Manakov N L and Rapoport L P 1971 Zh. Eksp. Teor. Fiz. 61968 (Engl. transl. 1972 Sov. Phys.-JETP 34 515)

Lambropoulos P 1972 Phys. Rev. Lett. 28585

Klarsfeld S and Maquet A 1972 Phys. Rev. Lett. 2979

Lambropoulos P 1972 Phys. Rev. Lett. 29453

[4] Delone G A, Manakov N L, Preobrazhenskii M A and Rapoport L P 1976 Zh. Eksp. Teor. Fiz. 701234 (Engl. transl. 1976 Sov. Phys.-JETP 43 642)

[5] Manakov N L and Ovsiannikov V D 1980 Zh. Eksp. Teor. Fiz. 791769 (Engl. transl. 1980 Sov. Phys.-JETP 52 895)

[6] Nakanishi K, Berova N and Woody R W (ed) 1994 Circular Dichroism: Principles and Applications (New York: $\mathrm{VCH}$ )

[7] Pasteur L 1848 Ann. Chim. Phys. III 24442

[8] Cherepkov N A 1983 Adv. At. Mol. Phys. 19395

[9] Böwering N, Lischke T, Müller N, Khalil T and Heinzman U 2001 Phys. Rev. Lett. 861187

[10] Schönhense G and Hornes J 1996 VUV and Soft-X-ray Photoionization ed U Becker and D A Shirley (New York: Plenum) p 607

[11] Manakov N L 1996 Super-Intense Laser-Atom Physics IV (NATO ASI Series: Series 3 vol 313) ed H G Muller and M V Fedorov (Dordrecht: Kluwer) p 153

[12] Manakov N L, Marmo S I and Meremianin A V 1996 J. Phys. B: At. Mol. Opt. Phys. 292711

[13] Manakov N L 1996 Zh. Eksp. Teor. Fiz. 1101244 (Engl. transl. 1996 Sov. Phys.-JETP 83 685)

[14] Manakov N L 1994 Zh. Eksp. Teor. Fiz. 1061286 (Engl. transl. 1994 Sov. Phys.-JETP 79 696)

[15] Berakdar J and Klar H 1992 Phys. Rev. Lett. 691175

[16] Briggs J S and Schmidt V 2000 J. Phys. B: At. Mol. Opt. Phys. 33 R1

[17] Berakdar J and Klar H 2001 Phys. Rep. 340473

[18] Manakov N L, Marmo S I and Meremianin A V 1996 J. Electron. Spectrosc. Relat. Phenom. 79331 Manakov N L, Meremianin A V, Carney J P J and Pratt R H 2000 Phys. Rev. A 61032711

[19] Manakov N L, Meremianin A V, Maquet A and Carney J P J 2000 J. Phys. B: At. Mol. Opt. Phys. 334425

[20] Fainshtein A G, Manakov N L and Marmo S I 1994 Phys. Lett. A 195358 Manakov N L, Marmo S I and Fainshtein A G 1995 Zh. Eksp. Teor. Fiz. 1081569 (Engl. transl. 1995 Sov. Phys.-JETP 81 860)

[21] Rapoport L P and Kornev A S 2000 J. Phys. B: At. Mol. Opt. Phys. 3387

[22] Manakov N L, Marmo S I and Volovich V V 1995 Phys. Lett. A 20442

Manakov N L, Marmo S I and Volovich V V 1996 J. Electron. Spectrosc. Relat. Phenom. 79327

[23] Fehr M, Berakdar J and Klar H 1994 J. Phys. B: At. Mol. Opt. Phys. 27 L401 Berakdar J, Engelns A and Klar H 1996 J. Phys. B: At. Mol. Opt. Phys. 291109

[24] Roller-Lutz Z, Wang Y, Lutz H O, Bastug T, Mukoyama T and Frike B 1999 Phys. Lett. A 26266

[25] Dorn A, Elliot A, Lower J, Weigold E, Berakdar J, Engelns A and Klar H 1998 Phys. Rev. Lett. 80257

[26] Lower J, Elliott A, Weigold E, Mazevet S and Berakdar J 2000 Phys. Rev. A 62012706

[27] Manakov N L and Ovsiannikov V D 1997 J. Phys. B: At. Mol. Opt. Phys. 302109

[28] Manakov N L, Ovsiannikov V D and Starace A F 1999 Phys. Rev. Lett. 824791

[29] Manakov N L, Frolov M V, Starace A F and Fabrikant I I 2000 J. Phys. B: At. Mol. Opt. Phys. 33 R141

[30] Manakov N L and Ovsiannikov V D 2000 J. Phys. B: At. Mol. Opt. Phys. 332466

[31] Manakov N L and Meremianin A V 1997 Zh. Eksp. Teor. Fiz. 1121984 (Engl. transl. 1997 Sov. Phys.-JETP 84 1080)

[32] Taieb R, Véniard V, Maquet A, Manakov N L and Marmo S I 2000 Phys. Rev. A 62013402

[33] Fifirig M and Florescu V 1998 Eur. Phys. J. D 2143

[34] Manakov N L and Ovsiannikov V D 2000 Laser Phys. 101251 
[35] Cionga A, Ehlotzky F and Zloh G 2000 Phys. Rev. A 61063417

[36] Cionga A, Ehlotzky F and Zloh G 2000 Phys. Rev. A 62063406

[37] Borca B, Flegel A V, Frolov M V, Manakov N L, Milošević D B and Starace A F 2000 Phys. Rev. Lett. 85732

[38] Saliéres P, L’Huillier A, Antoine P and Lewenstein M 1999 Adv. At. Mol. Opt. Phys. 4183

[39] Popov V S 2000 Zh. Eksp. Teor. Fiz. 11856 (Engl. transl. 2000 Sov. Phys.-JETP 91 48)

[40] Goreslavskii S P and Popruzhenko S V 1996 Zh. Eksp. Teor. Fiz. 1101200 (Engl. transl. 1996 Sov. Phys.-JETP 83663 )

Goreslavskii S P and Popruzhenko S V 1996 Laser Phys. 6780

[41] Mur V D, Popruzhenko S V and Popov V S 2001 Zh. Eksp. Teor. Fiz. 119893 (Engl. transl. 2001 Sov. Phys.JETP 92 777)

[42] Bashkansky M, Bucksbaum P H and Schumacher D W 1988 Phys. Rev. Lett. 602458

[43] Paulus G G, Zacher F, Walter H, Lohr A, Becker W and Kleber M 1998 Phys. Rev. Lett. 80484

[44] Paulus G G, Grasbon F, Dreischuh A, Walter H, Kopold R and Becker W 2000 Phys. Rev. Lett. 843791

[45] Goreslavskii S P, Popruzhenko S V and Shvetsov-Shilovski N I 2003 Laser Phys. at press

[46] Kopold R, Milošević D B and Becker W 2000 Phys. Rev. Lett. 843831

[47] Basile S, Trombetta F and Ferrante G 1988 Phys. Rev. Lett. 612435

Lambropoulos P and Tang X 1988 Phys. Rev. Lett. 612506

Muller H G, Petite G and Agostini P 1988 Phys. Rev. Lett. 612507

[48] Krstić P and Mittleman M H 1991 Phys. Rev. A 445938

[49] Manakov N L, Maquet A, Marmo S I, Véniard V and Ferrante G 1999 J. Phys. B: At. Mol. Opt. Phys. 323747

[50] Fifirig M and Cionga A 2002 J. Phys. B: At. Mol. Opt. Phys. 35821

Cionga A, Fifirig M and Ehlotzky F 2002 J. Phys. B: At. Mol. Opt. Phys. 354875 Fifirig M 2002 Phys. Scr. 65213

[51] Jaroń A, Kamiński J Z and Ehlotzky F 1999 Opt. Commun. 163115

[52] Wang Z M and Elliot D S 2000 Phys. Rev. Lett. 843795 Wang Z M and Elliot D S 2000 Phys. Rev. A 62053404

[53] Hall J, Robinson E and Branscomb L 1965 Phys. Rev. Lett. 141013

[54] Voronov G S, Delone G A, Delone N B and Kudrevatova O V 1965 Zh. Eksp. Teor. Fiz. Pis. Red. 2377 (Engl. transl. 1965 JETP Lett. 2 237)

Voronov G S and Delone N B 1966 Zh. Exp. Teor. Fiz. 5078 (Engl. transl. 1966 Sov. Phys.-JETP 23 54)

[55] Trainham R, Fletcher G D and Larson D J 1987 J. Phys. B: At. Mol. Phys. 20 L777

[56] Kwon N, Armstrong P S, Olsson T, Trainham R and Larson D J 1989 Phys. Rev. A 40676

[57] Blondel C, Champeau R-J, Crance M, Crubellier A, Delsart C and Marinescu D 1989 J. Phys. B: At. Mol. Opt. Phys. 221335

[58] Blondel C and Trainham R 1989 J. Opt. Soc. Am. B 61774

[59] Tang C Y, Harris P G, Mohagheghi A H, Bryant H C, Quick C R, Donahue J B, Reeder R A, Cohen S, Smith W W and Stewart J E 1989 Phys. Rev. A 396068

[60] Stapelfeldt H, Brink C and Haugen H K 1991 J. Phys. B: At. Mol. Opt. Phys. 24 L437

[61] Blondel C, Crance M, Delsart C, Giraud A and Trainham R 1990 J. Phys. B: At. Mol. Opt. Phys. 23 L685

[62] Blondel C, Crance M, Delsart C and Giraud A 1992 J. Physique 2839

[63] Blondel C, Crance M, Delsart C and Giraud A 1991 J. Phys. B: At. Mol. Opt. Phys. 243575

[64] Stapelfeldt H, Balling P, Brink C and Haugen H K 1991 Phys. Rev. Lett. 671731

[65] Davidson M D, Muller H G and van Linden van den Heuvell H B 1991 Phys. Rev. Lett. 671712

Davidson M D, Schumacher D V, Bucksbaum P H, Muller H G and van Linden van den Heuvell H B 1992 Phys. Rev. Lett. 693459

Davidson M D, Broers D, Muller H G and van Linden van den Heuvell H B 1992 J. Phys. B: At. Mol. Opt. Phys. 253093

[66] Tang C Y, Bryant H C, Harris P G, Mohagheghi A H, Reeder R A, Sharifian H, Toutounchi H, Quick C R, Donahue J B, Cohen S and Smith W W 1991 Phys. Rev. Lett. 663124

[67] Zhao X M, Gulley M S, Bryant H C, Strauss C E M, Funk D J, Stintz A, Rislove D C, Kyrala G A, Ingalis W B and Miller W A 1997 Phys. Rev. Lett. 781656

Gulley M S, Zhao X M, Bryant H C, Strauss C E M, Funk D J, Stintz A, Rislove D C, Kyrala G A, Ingalis W B and Miller W A 1999 Phys. Rev. A 604753

[68] Praestegaard L, Andersen T and Balling P 1999 Phys. Rev. A 593154

[69] Balling P, Andersen H H, Brodie C A, Pedersen U V, Petrunin V V, Raarup M K, Steiner P and Andersen T 2000 Phys. Rev. A 61022702

[70] Wigner E P 1948 Phys. Rev. 731002 
[71] Reichle R, Helm H and Kiyan I Yu 2001 Phys. Rev. Lett. 87243001

[72] Sturrus W G, Ratliff L P and Larson D J 1992 J. Phys. B: At. Mol. Opt. Phys. 25 L359

[73] Blondel C and Delsart C 1993 Nucl. Instrum. Methods Phys. Res. B 79156

[74] Blondel C and Delsart C 1993 Laser Phys. 33

[75] Duleu F, Blondel C and Delsart C 1995 J. Phys. B: At. Mol. Opt. Phys. 283845

[76] Duleu F, Blondel C and Delsart C 1995 J. Phys. B: At. Mol. Opt. Phys. 283861

[77] Nikolopoulos L A A and Lambropoulos P 1997 Phys. Rev. A 563106

[78] Borca B, Frolov M V, Manakov N L and Starace A F 2001 Phys. Rev. Lett. 87133001

[79] Robinson E J and Geltman S 1967 Phys. Rev. 1534

[80] Adelman S A 1973 J. Phys. B: At. Mol. Phys. 61986

[81] Manakov N L, Preobrazhenskii M A, Rapoport L P and Fainshtein A G 1978 Zh. Eksp. Teor. Fiz. 751243 (Engl. transl. 1978 Sov. Phys.-JETP 48 626)

[82] Golovinskii P A and Zon B A 1981 Bull. Acad. Sci. USSR Phys. Ser. 4512

[83] Delone N B, Kiyan I Yu, Krainov V P and Tugushev V I 1985 Opt. Spektrosk. 58262 (Engl. transl. 1985 Opt. Spectrosc. (USSR) $\mathbf{5 8}$ 157)

[84] Arrighini G P, Guidotti C and Durante N 1987 Phys. Rev. A 351528

[85] Gao B and Starace A F 1990 Phys. Rev. A 425580

[86] Geltman S 1990 Phys. Rev. A 426958

[87] Geltman S 1991 Phys. Rev. A 434930

[88] Liu C R, Gao B and Starace A F 1992 Phys. Rev. A 465985

[89] Fink M G J and Zoller P 1985 J. Phys. B: At. Mol. Phys. 18 L373

[90] Crance M and Aymar M 1985 J. Phys. B: At. Mol. Phys. 183529 Crance M 1988 J. Phys. B: At. Mol. Opt. Phys. 213559 Crance M 1990 J. Phys. B: At. Mol. Opt. Phys. 23 L285

[91] Shakeshaft R and Tang X 1987 Phys. Rev. A 363193

[92] Mercouris Th and Nicolaides C A 1988 J. Phys. B: At. Mol. Opt. Phys. 21 L285 Mercouris Th and Nicolaides C A 1991 J. Phys. B: At. Mol. Opt. Phys. 24 L557

[93] Jiang T F and Starace A F 1988 Phys. Rev. A 382347

[94] Pan C, Gao B and Starace A F 1990 Phys. Rev. A 416271

[95] Golovinski P A and Kiyan I Yu 1990 Usp. Fiz. Nauk 16097 (Engl. transl. 1990 Sov. Phys.-Usp. 33 453)

[96] Mu X 1990 Phys. Rev. A 422944

Mu X, Ruscheinski J and Graseman B 1990 Phys. Rev. A 422949

[97] Dörr M, Potvliege R M, Proulx D and Shakeshaft R 1990 Phys. Rev. A 424138

[98] Pan C and Starace A F 1991 Phys. Rev. A 44324

[99] Mercouris Th and Nicolaides C A 1992 Phys. Rev. A 452116

[100] Proulx D and Shakeshaft R 1992 Phys. Rev. A 46 R2221

[101] Collins L A and Merts A L 1992 Phys. Rev. A 456615

[102] Laughlin C and Chu S I 1993 Phys. Rev. A 484654

[103] Proulx D, Pont M and Shakeshaft R 1994 Phys. Rev. A 491208

[104] Dimou L and Faisal F H M 1994 J. Phys. B: At. Mol. Opt. Phys. 27 L333

[105] Van der Hart H W 1994 Phys. Rev. A 502508

[106] Wang J, Chu S I and Laughlin C 1994 Phys. Rev. A 503208

[107] Sánchez I, Bachau H and Martin F 1995 J. Phys. B: At. Mol. Opt. Phys. 282863 Sánchez I, Bachau H and Martin F 1997 J. Phys. B: At. Mol. Opt. Phys. 302417 Sánchez I, Bachau H and Martin F 1998 J. Phys. B: At. Mol. Opt. Phys. 31 L801

[108] Dörr M, Purvis J, Terao-Dunseath M, Burke P G, Joachain C J and Noble C J 1995 J. Phys. B: At. Mol. Opt. Phys. 284481

[109] Van der Hart H W 1996 J. Phys. B: At. Mol. Opt. Phys. 293059

[110] Yang B, Pont M, Shakeshaft R, Van Duijn E and Piraux B 1997 Phys. Rev. A 564946

[111] Scrinzi A and Piraux B 1998 Phys. Rev. A 581310

[112] Glass D H, Burke P G, Noble C J and Wöste G B 1998 J. Phys. B: At. Mol. Opt. Phys. 31 L667

[113] Nikolopoulos L A A and Lambropoulos P 1999 Phys. Rev. Lett. 823771

[114] Gribakin G F, Ivanov V K, Korol A V and Kuchiev M Yu 1999 J. Phys. B: At. Mol. Opt. Phys. 325463 Gribakin G F, Ivanov V K, Korol A V and Kuchiev M Yu 2000 J. Phys. B: At. Mol. Opt. Phys. 33821

[115] Pazdzersky V A, Usachenko V I and Ushnurtsev A V 2000 J. Phys. B: At. Mol. Opt. Phys. 331135

[116] Van der Hart H W 2000 J. Phys. B: At. Mol. Opt. Phys. 331789

[117] Masili M and Starace A F 2000 Phys. Rev. A 62033403

[118] Vinci N, Glass D H, Taylor K T and Burke P G 2000 J. Phys. B: At. Mol. Opt. Phys. 334799 
[119] Lagmago Kampta G, Grosges T, Piraux B, Hasbani R, Cormier E and Bachau H 2001 J. Phys. B: At. Mol. Opt. Phys. 34857

[120] Bachau H, Cormier E, Decleva P, Hansen J E and Martin F 2001 Rep. Prog. Phys. 641815

[121] Telnov D A and Chu S I 2002 Phys. Rev. A 66043417

[122] Nicolaides C A, Haritos C and Mercouris Th 2000 J. Phys. B: At. Mol. Opt. Phys. 332733

[123] Haritos C, Mercouris Th and Nicolaides C A 2001 Phys. Rev. A 63013410

[124] Telnov D A and Chu S I 1994 Phys. Rev. A 504099

[125] Telnov D A and Chu S I 1996 J. Phys. B: At. Mol. Opt. Phys. 294401

[126] Telnov D A and Chu S I 1999 Phys. Rev. A 592864 Telnov D A and Chu S I 2000 Phys. Rev. A 61013408

[127] Manakov N L and Fainshtein A G 1979 Dokl. Akad. Nauk. SSSR 244567 (Engl. transl. 1979 Sov. Phys.-Dokl. 24 41)

Manakov N L and Fainshtein A G 1980 Zh. Eksp. Teor. Fiz. 79751 (Engl. transl. 1980 Sov. Phys.-JETP 52 382)

[128] Gribakin G F and Kuchiev M Yu 1997 Phys. Rev. A 553760

[129] Kuchiev M Yu and Ostrovsky V N 1998 J. Phys. B: At. Mol. Opt. Phys. 312525

[130] Gribakin G F and Kuchiev M Yu 1997 J. Phys. B: At. Mol. Opt. Phys. 30 L657

[131] Manakov N L and Rapoport L P 1975 Zh. Eksp. Teor. Fiz. 69842 (Engl. transl.1975 Sov. Phys.-JETP 42 430) Berson I J 1975 J. Phys. B: At. Mol. Phys. 83078

[132] Becker W, Long S and McIver J K 1994 Phys. Rev. A 301540

[133] Lohr A, Kleber M, Kopold R and Becker W 1997 Phys. Rev. A 55 R4003

[134] Manakov N L, Frolov M V, Borca B and Starace A F 2000 Zh. Eksp. Teor. Fiz. Pis. Red. 72426 (Engl. transl 2000 JETP Lett. 72 294)

[135] Borca B, Frolov M V, Manakov N L and Starace A F 2002 Phys. Rev. Lett. 88193001

[136] Borca B, Starace A F, Flegel A V, Frolov M V and Manakov N L 2002 Phys. Rev. A 65 051402(R)

[137] Manakov N L, Starace A F, Flegel A V and Frolov M V 2002 Zh. Eksp. Teor. Fiz. Pis. Red. 76316 (Engl. transl. 2002 JETP Lett. 76 258)

[138] Berestetskii V B, Lifshitz E M and Pitaevskii L P 1982 Quantum Electrodynamics 2nd edn (Oxford: Pergamon) section 8

[139] Demkov Yu N and Ostrovsky V N 1988 Zero-Range Potentials and Their Applications in Atomic Physics (New York: Plenum)

[140] Du M L and Delos J B 1988 Phys. Rev. A 385609

[141] Manakov N L, Ovsiannikov V D and Rapoport L P 1986 Phys. Rep. 141319

[142] Landau L D and Lifshitz E M 1992 Quantum Mechanics 4th edn (Oxford: Pergamon)

[143] Manakov N L and Fainshtein A G 1981 Teor. Mat. Fiz. 48375 (Engl. transl. 1982 Theor. Math. Phys. 48 815)

[144] Potvliege R M and Shakeshaft R 1992 Atoms in Intense Laser Fields: Adv. At. Mol. Opt. Phys. (Suppl.) 1373

[145] Kramers H A 1956 Collected Scientific Papers (Amsterdam: North-Holland) Henneberger W C 1968 Phys. Rev. Lett. 21838

[146] Becker W, Long S and McIver J K 1990 Phys. Rev. A 424416

[147] Reiss H R 1980 Phys. Rev. A 221786

[148] Baz' A I, Zel'dovich Ya B and Perelomov A M 1971 Scattering, Reactions and Decays in Nonrelativistic Quantum Mechanics 2nd edn (Moscow: Nauka)

[149] Manakov N L, Marmo S I and Fainshtein A G 1986 Zh. Eksp. Teor. Fiz. 9151 (Engl. transl. 1986 Sov. Phys.JETP 64 29)

[150] Telnov D A 1991 J. Phys. B: At. Mol. Opt. Phys. 242967

[151] Broad J T and Reinhardt W P 1976 Phys. Rev. A 142159

[152] Yang C N 1948 Phys. Rev. 74764

[153] Telnov D A and Chu S I 2002 Phys. Rev. A 66063409

[154] Fedorov M V and Movsesian A M 1988 J. Phys. B: At. Mol. Opt. Phys. 21 L155

[155] Pont M and Gavrila M 1990 Phys. Rev. Lett. 652362

[156] Su Q, Eberly J H and Javananien J 1990 Phys. Rev. Lett. 64862

[157] Dörr M, Potvliege R and Shakeshaft R 1990 Phys. Rev. Lett. 642003

[158] Fedorov M V 1999 Laser Phys. 9209

[159] Gavrila M 2002 J. Phys. B: At. Mol. Opt. Phys. 35 R147

[160] Dondera M, Muller H G and Gavrila M 2002 Phys. Rev. A 65 031405(R)

[161] de Boer M P, Hoogenraad J H, Vrijen R B, Constantinescu R C, Noordam L D and Muller H G 1993 Phys. Rev. Lett. 713263

van Druten N J, Constantinescu R C, Schins J M, Nieuwenhuize H and Muller H G 1997 Phys. Rev. A 55622 
[162] Eberly J H, Grobe R, Law C K and Su Q 1992 Atoms in Intense Laser Fields ed M Gavrila (New York: Academic) p 301

Su Q 1993 Laser Phys. 2241

[163] Dörr M and Potvliege R M 2000 J. Phys. B: At. Mol. Opt. Phys. 33 L233

[164] Grozdanov T, Krstić P S and Mittelman M H 1990 Phys. Lett. A 149144

[165] Geltman S 1992 Phys. Rev. A 455293

[166] Krainov V P and Preobrazhenskii M A 1993 Zh. Exp. Teor. Fiz. 1031142 (Engl. transl. 1993 Sov. Phys.-JETP 76 559)

[167] LaGattuta K J 1994 Phys. Rev. A 491745

[168] Kamiński J Z 1995 Phys. Rev. A 524976

[169] Figueira C, Fring A and Schrader R 1999 Laser Phys. 9379

[170] Frolov M V, Manakov N L, Borca B and Starace A F 2001 J. Phys. B: At. Mol. Opt. Phys. 34 L579

[171] Potvliege R M and Shakeshaft R 1990 Phys. Rev. A 411609

[172] Langhoff P W, Epstein S T and Karplus M 1972 Rev. Mod. Phys. 44602

[173] Edwards M and Shakeshaft R 1988 Z. Phys. D 851

[174] Manakov N L, Frolov M V, Borca B and Starace A F 2001 Super-Intense Laser-Atom Physics (NATO Sci. Ser. II: Math., Phys. and Chemistry vol 12) ed B Piraux and K Rzążewski (Dordrecht: Kluwer) p 295

[175] Goldberger M L and Watson K M 1964 Collision Theory (New York: Wiley) section 8.2

[176] Piraux B and Potvliege R M 1998 Phys. Rev. A 575009

[177] Pont M, Shakeshaft R and Potvliege R M 1990 Phys. Rev. A 426969

[178] Pont M, Potvliege R M, Shakeshaft R and Teng Z-J 1992 Phys. Rev. A 458235

[179] Paulus G G, Nicklich W, Xu H, Lambropoulos P and Walther H 1994 Phys. Rev. Lett. 722851

[180] Hansch P, Walker M A and Van Woerkom L D 1997 Phys. Rev. A 55 R2535

[181] Hertlein M P, Bucksbaum P H and Muller H G 1997 J. Phys. B: At. Mol. Opt. Phys. 30 L197

[182] Muller H G and Kooiman F C 1998 Phys. Rev. Lett. 811207

[183] Paulus G G, Grasbon F, Walther H, Kopold R and Becker W 2001 Phys. Rev. A 64 021401(R)

[184] Kopold R, Becker W, Kleber M and Paulus G G 2002 J. Phys. B: At. Mol. Opt. Phys. 35217

[185] Popruzhenko S V, Korneev Ph A, Goreslavski S P and Becker W 2002 Phys. Rev. Lett. 89023001

[186] Newton R G 1982 Scattering Theory of Waves and Particles 2nd edn (New York: Springer)

[187] Baz’ A I 1957 Zh. Eksp. Teor. Fiz. 33923 (Engl. transl. 1958 Sov. Phys.-JETP 6 709)

[188] Adelman S A 1972 Phys. Rev. A 5508

[189] Pipin J and Bishop D M 1992 J. Phys. B: At. Mol. Opt. Phys. 2517

[190] Nicolaides C A, Mercouris Th and Piangos N A 1990 J. Phys. B: At. Mol. Opt. Phys. 23 L669

[191] Frolov M V, Manakov N L and Starace A F 2001 Phys. Rev. A 64023417

[192] Watson D K 1986 Phys. Rev. A 341016 Rescigno T N and McCurdy C W 1986 Phys. Rev. A 341882

[193] Mur V D, Pozdnyakov S G, Popov V S and Popruzhenko S V 2002 Zh. Eksp. Teor. Fiz. Pis. Red. 75294 (Engl. transl. 2002 JETP Lett. $\mathbf{7 5}$ 249)

[194] Zel'dovich Ya B 1961 Zh. Eksp. Teor. Fiz. 39776 (Engl. transl. 1961 Sov. Phys.-JETP 12 542)

[195] Krause J L, Schaffer K J and Kulander K C 1992 Phys. Rev. Lett. 683535

[196] van Linden van den Heuvell H B and Muller H G 1988 Multiphoton Processes (Studies in Modern Optics vol 8) ed S J Smith and P L Knight (Cambridge: Cambridge University Press) p 25 